Raízes de aplicações de superfícies em S2v...vS2vS1

Northon Canevari Leme Penteado 


\title{
Raízes de aplicações de superfícies em S2v...vS2vS1
}

\author{
Northon Canevari Leme Penteado
}

Orientador: Prof. Dr. Oziride Manzoli Neto

\begin{abstract}
Dissertação apresentada ao Instituto de Ciências Matemáticas e de Computação - ICMC-USP, como parte dos requisitos para obtenção do título de Doutor em Ciências - Matemática. VERSÃO REVISADA
\end{abstract}


Ficha catalográfica elaborada pela Biblioteca Prof. Achille Bassi e Seção Técnica de Informática, ICMC/USP, com os dados fornecidos pelo(a) autor(a)

Leme Penteado, Northon
Raízes de aplicações de superfícies em S2v...vS2vs1
/ Northon Leme Penteado; orientador Oziride
Manzoli Neto. -- São Carlos, 2015.
114 p.
Tese (Doutorado - Programa de Pós-Graduação em
Matemática) -- Instituto de Ciências Matemáticas e
de Computação, Universidade de São Paulo, 2015.
1. Teoria de Raízes. 2. Teoria de Homotopia. 3.
Superfícies. I. Manzoli Neto, Oziride, orient. II.
Título.




\section{Agradecimentos}

A Deus pela vida e saúde.

A minha família que sempre está do meu lado me apoiando e ensinando a ter confiança na vida, proporcionando muitas alegrias. Agradeço também a Cristiane Rodrigues e sua família que me acolheram com muito carinho fazendo parte, de modo inesquecível, de momentos felizes da minha vida.

Ao meu orientador Prof. Dr. Oziride Manzoli Neto, pela sincera amizade, pelos conselhos, pela sua contagiante paixão a matemática, pelo seu exemplo de dedicação ao trabalho e pelo sua constante disposição em ajudar nos problemas surgidos no decorrer da tese.

Aos meus companheiros de toda hora de Leme. Aos amigos e colegas de estudos, por tudo que sorrimos e sofremos juntos, tanto os de graduação quanto os de pós graduação. E também aos amigos de repúblicas.

Ao Instituto de Ciências Matemáticas e de Computação pela colaboração e proporcionar a excelente estrutura para a pós graduação. Agradeço também ao Coordenação de Aperfeiçoamento de Pessoal de Nível Superior (CAPES) e ao Conselho Nacional de Desenvolvimento Científico e Tecnológico (CNPq), pelos suportes e as concessões das bolsas. 


\section{Resumo}

Este trabalho é um estudo do problemas de raízes para aplicações $f: S \rightarrow W_{n}$, onde $S$ é uma superfície compacta, conexa e sem bordo e $W_{n}$ é o espaço obtido pela reunião em um ponto do círculo $S^{1}$ com $n$ esferas $S^{2}$. 


\section{Abstract}

The propose of this work is to studies the root problem for maps $f: S \rightarrow W_{n}$, where $S$ is a closed, connected, compact surface and $W_{n}$ is the space obtained by the one point union of a circle $S^{1}$ and $n$ spheres $S^{2}$. 



\section{Sumário}

Introdução

1 Representação de aplicações de superfícies orientáveis em W 5

1.1 Representacãão das classes de homotopia de aplicacõos de $S^{2} \mathrm{em} \mathrm{W} \ldots . \quad 5$

1.2 Representação das classes de homotopia de aplicações de $T^{2} \mathrm{em} W \ldots .22$

1.3 Representação das classes de homotopia de aplicações de $T_{g}$ em $W \ldots \ldots$

2 Representação de aplicações de superfícies orientáveis em $W_{n}$ 45

2.1 Representação das classes de homotopia de aplicações de $S^{2} \mathrm{em} W_{n} \ldots \ldots 45$

2.2 Representação das classes de homotopia de aplicaccões de $T^{2} \mathrm{em} W_{n}$. . . . 49

2.3 Representação das classes de homotopia de aplicações de $T_{g}$ em $W_{n} \ldots \ldots$

3 Representação de aplicações de superfícies não orientáveis em W $\quad 59$

3.1 Representação das classes de homotopia de aplicacõos de $\mathbb{R} P^{2} \mathrm{em} \mathrm{W}$... 59

3.2 Representação das classes de homotopia de aplicações de $\mathbb{R} P_{9}^{2} \mathrm{em} W \ldots 62$

4 Representação de aplicações de superfícies não orientáveis em $W_{n} \quad 69$

4.1 Representação das classes de homotopia de aplicações de $\mathbb{R} P^{2} \mathrm{em} W_{n}$. . . 69

4.2 Representação das classes de homotopia de aplicações de $\mathbb{R} P_{9}^{2}$ em $W_{n} \ldots 71$

5 Raízes de aplicações de superfícies orientáveis em $\left.W_{n}\right] \quad 75$

5.1 Raízes de aplicaconos de $S^{2}$ em $W_{n} \ldots \ldots \ldots \ldots \ldots \ldots \ldots \ldots$

5.2 Raízes de aplicações de $T^{2}$ em $W_{n} \ldots \ldots \ldots \ldots \ldots \ldots$ 
5.3 Raízes de aplicações de $T_{g}$ em $W_{n} \ldots \ldots \ldots$. . . . . . . . . . . . 83

6 Raízes de aplicacões de superfícies não orientáveis em $W_{n} \quad 85$

6.1 Raízes de aplicações de $\mathbb{R} P^{2}$ em $W_{n} \ldots \ldots \ldots \ldots$. . . . . . . . . 85

6.2 Raízes de aplicacões de $\mathbb{R} P_{9}^{2}$ em $W_{n} \ldots \ldots \ldots \ldots$. . . . . . 87

6.3 A sequência de Puppe-Barrat no caso $\left[T^{2} ; W\right]$ e alguns resultados . . . . . 103 


\section{Introdução}

Dados uma aplicação $f: X \rightarrow Y$ entre dois espaços topológicos e $y \in Y$, então $f^{-1}(y)=\{x \in X: f(x)=y\}$ é o conjunto de raízes de $f$ em $y$. Um dos problemas da Teoria de Raízes é determinar qual é o conjunto minimal de raízes para um dado $y \in Y$, isto é, determinar, a menos de homotopia, qual é o menor conjunto de raízes de um dado $y \in Y$. Outro problema é estudar a existência de uma aplicação $g: X \rightarrow Y$, tal que $g$ é homotópica a aplicação $f$, e $g$ realiza o conjunto minimal de raízes para um dado $y \in Y$, isto é, $g^{-1}(y)$ é o conjunto minimal de raízes de $y$.

No contexto em que $X$ e $Y$ são variedades, tem sido estudados em inúmeros trabalhos. Aniz, C. e Fenille, M. C. estudaram, em [1] e [10], teoria de raízes de aplicações $f: K \rightarrow$ $M$, onde $K$ é um CW-complexo e $\mathrm{M}$ é uma variedade, não necessariamente estes dois problemas de raízes citados acima.

Neste trabalho estamos interessados nestes dois problemas no caso em que $f: S \rightarrow$ $\underbrace{S^{2} \vee \ldots \vee S^{2}}_{n-\text { vezes }} \vee S^{1}$ é uma aplicação contínua, onde $S$ é uma superfície fechada, conexa e sem bordo e $\vee$ é a colagem por um ponto entre espaços topológicos. Para isso, a cada classe de homotopia $[f]$, será associado um polinômio proveniente de homologia com coeficientes locais. A partir de um polinômio é construída uma aplicação, denominada especial, que tem boas propriedades tal que o polinômio associado a classe de homotopia desta aplicação construída é o polinômio dado. E, para representar uma dada classe de homotopia $[f]$ de uma aplicação, mostra-se que existe uma aplicação especial $g \in[f]$. Por fim, é demonstrado que uma aplicação especial realiza o conjunto minimal de raízes, verificando que é uma representação com boas propriedades. 
O contra-domínio de $f$ será denotado por $W_{n}$ e quando $n=1$ apenas por $W$.

Este trabalho foi dividido em 6 capítulos e um apêndice.

Os livros [7], [9], 14], [16], [19] e [20] foram utilizados como apoio para os resultados e técnicas de teoria de topologia algébrica.

No primeiro capítulo é feita uma representação de classes de homotopia de aplicações de superfícies fechadas, conexas, orientáveis e sem bordo $S$ em $W$. Para isso este capítulo foi dividido em 3 seções.

Na primeira seção, a cada classe de homotopia de uma aplicação $f: S^{2} \rightarrow W$ é associado, utilizando homologia com coeficientes locais, um polinômio. Em seguida, é construída uma aplicação com boas propriedades a partir de um polinômio dado e esta aplicação é denominada especial. Por fim, dada uma classe de homotopia $[f]$ e usando técnicas geométricas é mostrado que existe aplicação $g: S^{2} \rightarrow W$ tal que $g \in[f]$ é uma aplicação especial.

Na segunda seção, é considerada aplicações $f: T^{2} \rightarrow W$. Recorrendo a primeira seção é associado um polinômio a classe de homotopias $[f]$ e seguindo a mesma linha de resultados da primeira seção é definido o que é uma aplicação especial neste caso e depois é mostrado que em cada classe de homotopia $[f]$ existe uma aplicação $g \in[f]$ que é especial.

Fazendo referências as seções anteriores, na terceira seção é associado um polinômio a classe de homotopia de aplicações $f: T_{g} \rightarrow W$, definido o que é uma aplicação especial neste caso e mostrado que existe uma aplicação especial em cada classe de homotopia $[f]$, onde $T_{g}$ é uma superfície compacta, conexa, sem bordo e orientável de gênus $g>1$.

O segundo capítulo tem três seções que consideram o $W_{n}$ como contra domínio de aplicações. Nas três seções é feito como nas seções do capítulo anterior, mas para aplicações $f: S^{2} \rightarrow W_{n}, f: T^{2} \rightarrow W_{n}$ e $f: T_{g} \rightarrow W_{n}$, respectivamente, onde $n>1$.

No terceiro capítulo são consideradas aplicações $f: \mathbb{R} P_{g}^{2} \rightarrow W$, onde $\mathbb{R} P_{g}^{2}$ é a notação para soma conexa de $g$ planos projetivos dois dimensionais, para algum $g$ finito. Para associar polinômios às classes de homotopias destas aplicações são utilizados os espaços de recobrimento duplo orientáveis de $\mathbb{R} P_{g}^{2}$, que são as superfícies $S^{2}$ ou $T_{g}$. Logo, para definir a aplicação especial e para provar as existências de aplicações especiais recai-se nos 
casos das seções anteriores. Na primeira seção é considerado $g=1$ e na segunda seção é considerado $g>1$.

No quarto capítulo é feita a representação de classes de aplicações $f: \mathbb{R} P^{2} \rightarrow W_{n}$ e $f: \mathbb{R} P_{g}^{2} \rightarrow W_{n}$ utilizando os recobrimentos duplos orientáveis.

O quinto e sexto capítulos são sobre minimalidade de raízes de uma aplicação. Nestes capítulos é mostrado que as aplicações especiais, definidas nas seções anteriores, são as aplicações que realizam os conjuntos de raízes minimais em cada caso. O quinto capítulo é dividido em três seções para tratar de minimalidades de raízes de aplicações de superfícies compactas, conexas, sem bordo e orientáveis em $W_{n}$, sendo a primeira seção, trata-se de raízes de aplicações $f: S^{2} \rightarrow W_{n}$, a segunda seção trata-se de raízes de aplicações $f: T^{2} \rightarrow W_{n}$ e a terceira seção trata-se de raízes de aplicações $f: T_{g} \rightarrow W_{n}$. O sexto capítulo é dividido em duas seções para tratar de minimalidades de raízes de aplicações de superfícies compactas, conexas, sem bordo e não orientáveis em $W_{n}$, sendo a primeira seção, para tratar de raízes de aplicações $f: \mathbb{R} P^{2} \rightarrow W_{n}$ e a segunda seção para tratar de raízes de aplicações $f: \mathbb{R} P_{g}^{2} \rightarrow W_{n} \operatorname{com} g>1$.

Por último é feito um apêndice no qual, primeiramente, é dada uma introdução a Homologia com Coeficientes Locais e por seguinte é apresentado via teoria de homotopia a caracterização dos conjuntos das classes de homotopia que utilizamos. Nele é apresentada uma sequência exata chamada sequência de Puppe-Barrat, originária dos artigos [2], [3] e [4], que define uma ação do grupo $\left[S^{2} ; W\right]$ sobre o $[S ; W]$. Esta ação descreve as classes de homotopia e a partir disto é caracterizada cada classe de homotopia de aplicações de $S$ em $S^{2} \vee S^{1}$, resultando em certas bijeções entre $[S, W]$ e outros conjuntos que as caracterizam.

Para finalizar a introdução, algumas convenções: Todas as aplicações são consideradas contínuas. O conjunto de classes de homotopias livres de aplicações de $X$ em $Y$ é denotado por $[X, Y]$. O espaço $W_{n}$ considerado é o espaço tal que é tomado $\left\{p_{1}, \ldots, p_{n}\right\}$ pontos, dois a dois distintos, de uma esfera um dimensional $S^{1}$ e $n$ esferas dois dimensionais $S_{i}^{2}$, duas a duas disjuntas com $S_{i}^{2} \cap S^{1}=\emptyset$ para todo $i \in\{1, \ldots, n\}$. O espaço $W_{n}$ é o espaço obtido pela colagem, pelo ponto $p_{i}$, de cada $S_{i}^{2}$ em $S^{1}$, portanto, $W_{n}$ tem mesmo tipo de homotopia que $\underbrace{S^{2} \vee \ldots \vee S^{2}}_{n \text {-vezes }} \vee S^{1}$. Por último, para denotar homotopia de aplicações ou equivalências de espaços topológicos usaremos $\cong$ e para denotar isomorfismo entre objetos 
algébricos usaremos $\simeq$. 


\section{Capítulo}

1

\section{Representação de aplicações de superfícies orientáveis em $W$}

\subsection{Representação das classes de homotopia de aplica-} ções de $S^{2}$ em $W$

Nesta seção usaremos homologia com coeficientes locais para encontrar um polinômio associado a cada classe de homotopia de aplicações de $S^{2}$ em $W$, que representarão as classes de homotopia destas aplicações.

Calcularemos a homologia com coeficientes locais $H_{*}\left(W, \mathbb{Z} \pi_{\rho}\right)$ e será dada uma interpretação geométrica a estes módulos e o homomorfismo induzido por $f$ no segundo grupo de homologia.

\section{Polinômio associado a uma classe de homotopia}

Consideremos $\pi_{1}(W)=\pi_{1}\left(S^{2} \vee S^{1}\right) \simeq \mathbb{Z}<t>($ gerador por $t), \mathbb{Z}\left[\pi_{1}(W)\right] \simeq \mathbb{Z}[\mathbb{Z}] \simeq$ $\mathbb{Z}\left[t, t^{-1}\right]$, onde a estrutura de $\mathbb{Z}\left[t, t^{-1}\right]$-módulo de $\mathbb{Z}\left[t, t^{-1}\right]$ é dada pelo homomorfismo $\rho_{\pi_{1}}: \pi_{1}(W) \rightarrow A u t_{\mathbb{Z}}\left(\mathbb{Z}\left[t, t^{-1}\right]\right)$ tal que $\rho_{\pi_{1}}(t)(\sigma)=t . \sigma$, ou seja, $\rho_{\pi_{1}}$ é a multiplicação por $t$.

Logo a homologia com coeficientes locais $H_{*}\left(W ; \mathbb{Z}\left[t, t^{-1}\right]_{\rho_{\pi_{1}}}\right)$ é calculada da seguinte 
forma:

1) $S_{*}\left(W, \mathbb{Z}\left[t, t^{-1}\right]\right)=S_{*}(\widetilde{W}) \otimes_{\mathbb{Z}\left[t, t^{-1}\right]} \mathbb{Z}\left[t, t^{-1}\right]=S_{*}(\widetilde{W})$, onde $S_{*}(\widetilde{W})$ tem estrutura de $\mathbb{Z}\left[t, t^{-1}\right]$-módulo natural e $\widetilde{W}$ é o recobrimento universal de $W$ ilustrado pelo figura abaixo

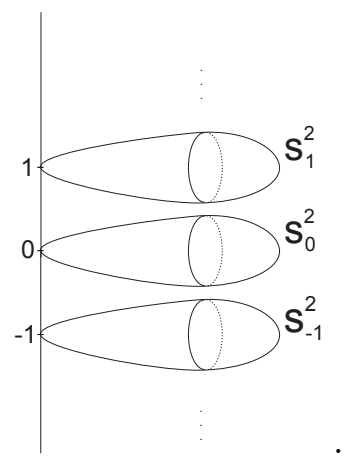

2) temos o complexo de cadeias

$$
\ldots S_{3}(\widetilde{W}) \stackrel{\widetilde{\partial_{3}}}{\longrightarrow} S_{2}(\widetilde{W}) \stackrel{\widetilde{\partial_{2}}}{\longrightarrow} S_{1}(\widetilde{W}) \stackrel{\widetilde{\partial_{1}}}{\longrightarrow} S_{0}(\widetilde{W}) \stackrel{\widetilde{\partial_{0}}}{\longrightarrow} 0
$$

Usando decomposição celular, temos $W=e_{0} \cup e_{1} \cup e_{2}$, onde $e_{0}=P, e_{1}=S^{1}$ e $e_{2}=S^{2}$. Fixando os levantamentos destas células como sendo $\widetilde{e}_{0}=0, \widetilde{e}_{1}=[0,1]$ e $\widetilde{e}_{2}=S_{0}^{2}$, temos a respectiva decomposição

$\widetilde{W}=\left(\ldots \cup \widetilde{e}_{0}^{t^{-2}} \cup \widetilde{e}_{0}^{t^{-1}} \cup \widetilde{e}_{0} \cup \widetilde{e}_{0}^{t} \cup \widetilde{e}_{0}^{t^{2}} \cup \ldots\right) \cup\left(\ldots \widetilde{e}_{1}^{t^{-2}} \cup \widetilde{e}_{1}^{t^{-1}} \cup \widetilde{e}_{1} \cup \widetilde{e}_{1}^{t} \cup \widetilde{e}_{1}^{t^{2}} \cup \ldots\right) \cup\left(\ldots \cup \widetilde{e}_{2}^{t^{-2}} \cup \widetilde{e}_{2}^{t^{-1}} \cup \widetilde{e}_{2} \cup \widetilde{e}_{2}^{t} \cup \widetilde{e}_{2}^{t^{2}} \cup \ldots\right)$,

onde $\widetilde{e}_{i}^{t^{j}}=t^{j} \widetilde{e}_{i}$ (lembrando que $S_{*}(\widetilde{W})$ é $\mathbb{Z}\left[t, t^{-1}\right]$-módulo). Utilizando a ilustração acima, geometricamente a multiplicação de $\widetilde{e}_{0}$ por $t^{j}$ é o ponto $j$ da reta, a multiplicação de $\widetilde{e}_{1}$ por $t^{j}$ é o segmento $[j, j+1]$ e a multiplicação de $\widetilde{e}_{2}$ por $t^{j}$ é a esfera $S_{j}^{2}$.

Portanto $S_{0}(\widetilde{W})=\mathbb{Z}\left[t, t^{-1}\right]$ gerado por $<\widetilde{e}_{0}>, S_{1}(\widetilde{W})=\mathbb{Z}\left[t, t^{-1}\right]$ gerado por $<\widetilde{e}_{1}>$, $S_{2}(\widetilde{W})=\mathbb{Z}\left[t, t^{-1}\right]$ gerado por $<\widetilde{e}_{2}>$ e $S_{i}(\widetilde{W})=0$ para $i>2$ e $i<0$.

Os operadores bordos $\widetilde{\partial}_{i}$ são dados por:

$\mathrm{i}=1: \widetilde{\partial}_{1}\left(\widetilde{e}_{1}\right)=\widetilde{e}_{0}^{t}-\widetilde{e}_{0}=t \widetilde{e}_{0}-\widetilde{e}_{0}=(t-1) \widetilde{e}_{0} . \log 0 \widetilde{\partial}_{1}=(t-1)$.

$\mathrm{i}=2: \widetilde{\partial}_{2}\left(\widetilde{e}_{2}\right)=0$. Logo $\widetilde{\partial}_{2}=0$

i $>2$ : para $i>2$, desde que $S_{i}(\widetilde{W})=0$, temos $\widetilde{\partial}_{i}=0$.

O complexo de cadeia é então 


$$
\ldots 0 \stackrel{\widetilde{\partial_{3}}=0}{\longrightarrow} \mathbb{Z}\left[t, t^{-1}\right] \stackrel{\widetilde{\partial_{2}}=0}{\longrightarrow} \mathbb{Z}\left[t, t^{-1}\right] \stackrel{\widetilde{\partial_{1}}=(t-1)}{\longrightarrow} \mathbb{Z}\left[t, t^{-1}\right] \stackrel{\widetilde{\partial_{0}}}{\longrightarrow} 0 .
$$

Logo temos a homologia

$$
H_{i}\left(W ; \mathbb{Z}\left[t, t^{-1}\right]_{\rho_{\pi_{1}}}\right) \simeq \begin{cases}\frac{\mathbb{Z}\left[t, t^{-1}\right]}{<(t-1)>} \cong \mathbb{Z} & \text { se } i=0 \\ \mathbb{Z}\left[t, t^{-1}\right] & \text { se } i=2 \\ 0 & \text { c.c }\end{cases}
$$

Seja $p: \widetilde{W} \rightarrow W$ o recobrimento universal. Pelo lema 6.2.1 temos o sistema de coeficientes locais sobre $W$ associado ao recobrimento $p$

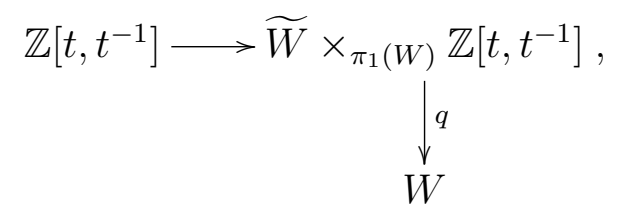

onde $q([\widetilde{w}, z])=p(\widetilde{w})$.

Dada uma aplicação $f: S^{2} \rightarrow W$ induzida de $f$ temos os homomorfismo induzido por $f$ em homologia com coeficientes locais. A estrutura de $\mathbb{Z}\left[t, t^{-1}\right]$-módulo de $\mathbb{Z}\left[t, t^{-1}\right]$ é determinada pela imagem da induzida da $f$ no grupo fundamental. Como $\pi_{1}\left(S^{2}\right)$ é trivial, a homologia com coeficientes locais $\mathbb{Z}\left[t, t^{-1}\right]$ de $S^{2}$ é a homologia usual com coeficientes em $\mathbb{Z}\left[t, t^{-1}\right]$.

Neste caso, a homologia $H_{*}\left(S^{2} ; \mathbb{Z}\left[t, t^{-1}\right]_{\rho_{\pi_{1}}^{*}}\right)$ é dada por

$$
H_{i}\left(S^{2} ; \mathbb{Z}\left[t, t^{-1}\right]_{\rho_{\pi_{1}}^{*}}\right) \simeq \begin{cases}\mathbb{Z}\left[t, t^{-1}\right] & \text { se } i=0,2 \\ 0 & \text { se } \text { c.c }\end{cases}
$$

onde $\rho_{\pi_{1}}^{*}$ é o "pullback"de $\rho_{\pi_{1}}$.

Seja $f_{i}: H_{i}\left(S^{2} ; \mathbb{Z}\left[t, t^{-1}\right]\right) \rightarrow H_{i}\left(W ; \mathbb{Z}\left[t, t^{-1}\right]_{\rho_{\pi_{1}}}\right)$ o homomorfismo induzido por $f$ em homologia com coeficientes locais.

Quando $i=2$, temos o homomorfismo $f_{2}: H_{2}\left(S^{2} ; \mathbb{Z}\left[t, t^{-1}\right]\right) \rightarrow H_{2}\left(W ; \mathbb{Z}\left[t, t^{-1}\right]_{\rho_{\pi_{1}}}\right)$, dada por $f_{2}([\sigma])=[f \circ \sigma]$, onde $f_{2}([\sigma])$ é da forma $a_{-i} t^{-i}+a_{-i+1} t^{-i+1}+\ldots+a_{0}+a_{1} t+$ $\ldots+a_{j} t^{j}$, onde $i, j \in \mathbb{N}$, desde que $H_{2}\left(W ; \mathbb{Z}\left[t, t^{-1}\right]_{\rho_{1}}\right) \simeq \mathbb{Z}\left[t, t^{-1}\right]$. 
Este elemento pressupõe feita uma escolha de isomorfismo $\Phi: H_{2}\left(W ; \mathbb{Z}\left[t, t^{-1}\right]_{\rho_{\pi_{1}}}\right) \rightarrow$ $\mathbb{Z}\left[t, t^{-1}\right]$ pois quando dizemos que $f_{2}(x)$ é desta forma estamos identificando com $\Phi\left(f_{2}(x)\right)$.

Seja $\Phi: H_{2}\left(W ; \mathbb{Z}\left[t, t^{-1}\right]_{\rho_{\pi_{1}}}\right) \rightarrow \mathbb{Z}\left[t, t^{-1}\right]$ um isomorfismo, isto significa que fixamos o ponto base $\widetilde{x}_{\Phi}$ do espaço $\widetilde{W}$. Suponha que fixamos o isomorfismo tal que o ponto base seja $0 \in \mathbb{Z} \subset \mathbb{R} \subset \widetilde{W}$.

Seja um elemento de $H_{2}\left(W ; \mathbb{Z}\left[t, t^{-1}\right]_{\rho_{\pi_{1}}}\right)$, isto é, $t^{-i} a_{-i}+t^{-i+1} a_{-i+1}+\ldots+t^{j-1} a_{j-1}+t^{j} a_{j}$, então cada monômio e seus coeficientes tem uma interpretação geométrica. Escolhemos uma orientação para as células $S_{i}^{2}$ do recobrimento universal $\widetilde{W}$. A representação geométrica deste elemento é feita em $\widetilde{W}$ e é como na parte vermelha da ilustração

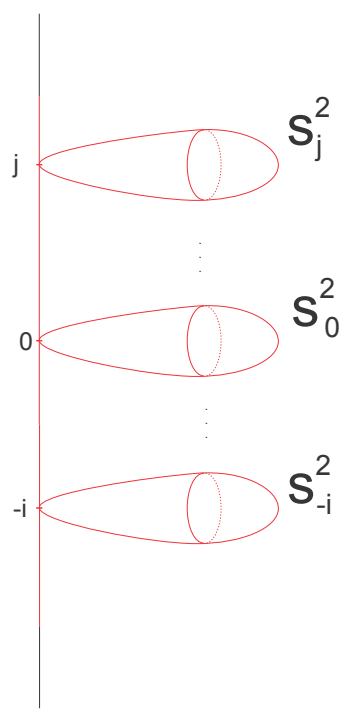

isto é, cada monômio $t^{j} a_{j}$ significa que a esfera $S_{j}^{2}$ é coberta $a_{j}$ vezes, isto é, a esfera $S_{j}^{2}$ é recoberta com grau $a_{j}$ de acordo com a orientação escolhida. Além disso o intervalo $[-i, j]$ também é coberto.

Observemos que esta associação de um elemento $t^{-i} a_{-i}+t^{-i+1} a_{-i+1}+\ldots+t^{j-1} a_{j-1}+$ $t^{j} a_{j} \in H_{2}\left(W ; \mathbb{Z}\left[t, t^{-1}\right]_{\rho_{\pi_{1}}}\right)$ a uma aplicação de $S^{2}$ em $W$ independente do representante da classe de homotopia desta aplicação, isto é, podemos fazer esta associação à classe de homotopia da aplicação. Portanto a representação geométrica pode ser como na ilustração acima na qual a parte vermelha contenha o intervalo $[-i, j]$. Mas, a menos de homotopia, a representação irá conter exatamente o intervalo $[-i, j]$ e o mesmo vale para as esferas $S_{j}^{2}$, isto é, elas são recobertas exatamente $a_{j}$ vezes de acordo com a orientação fixada anteriormente. 
Fazendo a imagem deste elemento pela aplicação $p$ temos que a imagem de $f$ recobre a esfera $S^{2}, a_{-i}+a_{-i+1}+\ldots+a_{j-1}+a_{j}$ vezes.

Portanto dada uma aplicação $f: S^{2} \rightarrow W$ e feita a escolha do isomorfismo $\Phi$ : $H_{2}\left(W ; \mathbb{Z}\left[t, t^{-1}\right]_{\rho_{\pi_{1}}}\right) \rightarrow \mathbb{Z}\left[t, t^{-1}\right]$, temos um elemento associado a $f$ que é $\Phi\left(f_{2}(\sigma)\right)$, onde $\sigma$ é um gerador de $H_{2}\left(S^{2} ; \mathbb{Z}\left[t, t^{-1}\right]_{\rho_{\pi_{1}}^{*}}\right) \simeq_{\Phi} \mathbb{Z}\left[t, t^{-1}\right]$.

Suponha que $t^{-i} a_{-i}+t^{-i+1} a_{-i+1}+\ldots+t^{j-1} a_{j-1}+t^{j} a_{j}$ seja o elemento associado a uma aplicação $f: S^{2} \rightarrow W$. Considere o elemento $t^{k}\left(t^{-i} a_{-i}+t^{-i+1} a_{-i+1}+\ldots+t^{j-1} a_{j-1}+t^{j} a_{j}\right)$, então a representação geométrica deste elemento será a mesma de $t^{-i} a_{-i}+t^{-i+1} a_{-i+1}+$ $\ldots+t^{j-1} a_{j-1}+t^{j} a_{j}$, mas transladado por $k$ em $\widetilde{W}$ como na figura

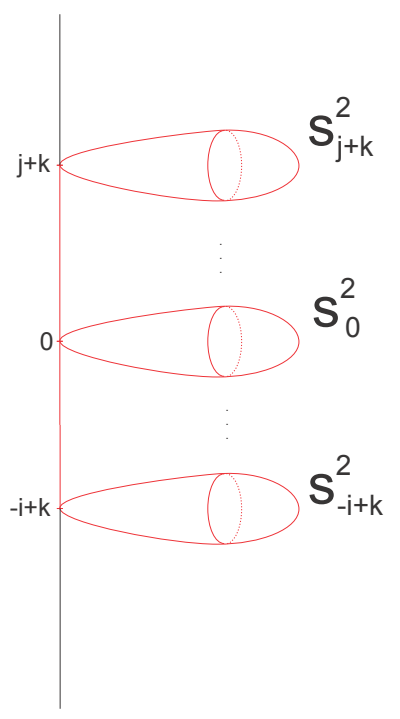

Se $t^{k}\left(t^{-i} a_{-i}+t^{-i+1} a_{-i+1}+\ldots+t^{j-1} a_{j-1}+t^{j} a_{j}\right)$ é o elemento associado a uma aplicação $g: S^{2} \rightarrow W$, então a imagem por $p$ das representações geométricas dos elementos $t^{-i} a_{-i}+$ $t^{-i+1} a_{-i+1}+\ldots+t^{j-1} a_{j-1}+t^{j} a_{j}$ e $t^{k}\left(t^{-i} a_{-i}+t^{-i+1} a_{-i+1}+\ldots+t^{j-1} a_{j-1}+t^{j} a_{j}\right)$ são as mesmas. Portanto a imagem de $f$ e $g$ são as mesmas também.

Além disso, como o conjunto $\left[S^{2}, W\right]$ é o conjunto de classes de aplicações por homotopias livres, então $g \in[f]$.

Dado um elemento de $\mathbb{Z}\left[t, t^{-1}\right]$ associado a uma aplicação $f: S^{2} \rightarrow W$, tal que tenha potência de $t$ negativa e a menor potência seja $-i$. Podemos multiplicar este elemento pelo inversível $t^{i}$ de $\mathbb{Z}\left[t, t^{-1}\right]$ e assim este novo elemento, que chamaremos de polinômio, terá somente potências positivas de $t$ e a menor potência de $t$ é 0 . Este polinômio será o polinômio associado a $[f]$ que terá somente potências de $t$ positivas, com $a_{0} \neq 0$. 
Correspondente ao polinômio nulo $\left(a_{0}=0\right)$ temos a aplicação homotópica a aplicação constante.

Lembrando que $[f]$ é a classe de homotopia livre da aplicação $f$, podemos definir:

Definição 1.1.1. Dados uma aplicação $f: S^{2} \rightarrow W$ e sua classe de homotopia. O polinômio nulo ou um polinômio com $a_{0} \neq 0$ e $n>0$, como construído acima é o polinômio associado a classe de homotopia $[f]$ que é denotado por $p_{[f]}(t)$.

\section{Construção de um bom representante}

Na seção anterior é associado à classe de homotopia de uma aplicação $f: S^{2} \rightarrow W$ um polinômio $p_{[f]}(t) \in H_{2}\left(W ; \mathbb{Z}[\mathbb{Z}] \rho_{\pi_{1}}\right)$.

Nesta seção será construída uma aplicação $f: S^{2} \rightarrow W$ a partir de um dado polinômio $q(t)=a_{0}+a_{1} t+\ldots+a_{j-1} t^{j-1}+a_{j} t^{j} \in \mathbb{Z}\left[t, t^{-1}\right]$ tal que $p_{[f]}(t)=q(t)$.

O espaço $\widetilde{W}$ pode ser descrito como $\mathbb{R} \cup\left(\bigcup_{j \in \mathbb{Z}} S_{j}^{2}\right)$ tal que para $j \in \mathbb{Z}, \mathbb{R} \cap S_{j}^{2}=\{j\} \subset$ $\mathbb{Z} \subset \mathbb{R}$

A função de recobrimento $p: \widetilde{W} \rightarrow W$ é tal que $\left.p\right|_{S_{j}^{2}}: S_{j}^{2} \rightarrow S^{2}$ é um difeomorfismo que preserva orientação.

Para qualquer $j \in \mathbb{Z},\left.p\right|_{[j, j+1]}:[j, j+1] \rightarrow W$ atinge $S^{1} \operatorname{com} p(j)=P=p(j+1)$ e $\left.p\right|_{(j, j+1)}:(j, j+1) \rightarrow W \backslash S^{2}$ é dado por $\left.p\right|_{(j, j+1)}(s)=e^{2 \pi i s}$, onde $P$ é identificado com $1 \in S^{1} \subset \mathbb{C}$ o plano complexo.

A ação de $\pi_{1}(W) \simeq \mathbb{Z}$ gerado por $t$ sobre $\widetilde{W}$ é tal que $t^{i} S_{j}^{2}=S_{j+i}^{2}$ e $t^{i}[j, j+1]=$ $[j+i, j+1+i]$.

Dado o polinômio $q(t)$, descreveremos uma aplicação $\widetilde{f}_{q}: S^{2} \rightarrow \widetilde{W}$ tal que a classe de homotopia de $f=p \circ \widetilde{f}_{q}$ corresponde ao polinômio $q(t)$.

A aplicação $\widetilde{f}_{q}: S^{2} \rightarrow \widetilde{W}$ é como na ilustração: 


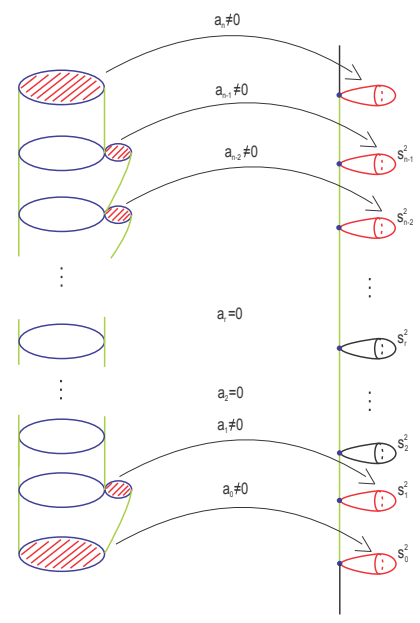

onde a imagem da parte vermelha são as esferas de cores vermelhas em $\widetilde{W}$, a imagem da parte verde é o intervalo verde de $\widetilde{W}$ e a imagem da parte azul são os pontos de coordenadas inteiras em azul de $\widetilde{W}$.

Além disso, cada disco vermelho atinge uma esfera com um grau $a_{i} \neq 0$ e cada esfera vermelha é atingida por somente um disco vermelho. A aplicação $\tilde{f}_{q}$ restrita a $S^{2}$ menos a parte vermelha é a projeção no intervalo verde.

Logo definimos a aplicação $f: S^{2} \rightarrow W$ por $p \circ \widetilde{f}_{q}$. Por construção $p_{[f]}(t)=q(t)$.

Definição 1.1.2. Uma aplicação $f: S^{2} \rightarrow W$ construída como acima a partir do polinômio $p_{[f]}(t)$ é chamada de aplicação especial.

\section{Construção de uma aplicação especial na classe de homotopia de $f: S^{2} \rightarrow W$}

Dada uma aplicação $f: S^{2} \rightarrow W$, usaremos algumas técnicas geométricas para encontrar uma aplicação $g: S^{2} \rightarrow W$ onde $g \in[f]$ e $g$ é especial.

Primeiramente note que se a imagem de $f$ esta contida em $S^{1} \subset W$, desde que $\pi_{2}\left(S^{1}\right)=$ 0 , temos $f$ homotópica a uma aplicação constante.

Se a imagem de $f$ esta contida em $S^{2} \subset W$, então o polinômio $p_{[f]}=\mathbb{O}$ e neste caso $f$ é homotópica a uma aplicação constante

Se $p_{[f]}=a_{0} \neq 0$ e a aplicação $f$ pode ser considerada como aplicação de $S^{2}$ em $S^{2}$ tal que $a_{0}$ é o seu grau. 
Observação: Uma aplicação $f: X \rightarrow Y$ entre dois espaços topológicos é dita fortemente sobrejetiva se para qualquer $g$ homotópica a $f, g$ é sobrejetora.

Logo daqui por adiante, consideremos aplicações de $S^{2}$ em $W$ fortemente sobrejetiva.

Os resultados seguintes modificarão $f$, através de homotopias, para uma aplicação especial. Durante este processo a aplicação modificada sempre será denotada por $f$, isto é, manteremos a mesma notação nos vários passos das demonstrações.

Lema 1.1.1. Seja uma aplicação $f: S^{2} \rightarrow W$ contínua fortemente sobrejetora. Então $f$ é homotópica a uma aplicação $g: S^{2} \rightarrow W$ tal que $g^{-1}\left(W \backslash S^{1}\right)$ é uma subvariedade de $S^{2}$ homeomorfa $a \bigsqcup_{t=1}^{n} B_{t}^{2}$, onde $n$ é finito, $B_{t}^{2}$ é o interior de discos $D_{t}^{2}$ tal que $\partial D_{t}^{2} \cong S_{t}^{1}$ e para $i \neq j, S_{i}^{1} \cap S_{j}^{1}=\emptyset$. Além disso, $g\left(\partial D_{t}^{2}\right)=P$ e $\left.g\right|_{\left(D_{t}^{2}, \partial D_{t}^{2}\right)}:\left(D_{t}^{2}, \partial D_{t}^{2}\right) \rightarrow\left(S^{2}, P\right)$ tem grau \pm 1 .

Demonstração: Primeiramente observe que como $S^{2}$ é variedade e $W$, a menos do ponto singular $P$, tem propriedades locais de variedades, podemos usar os conceitos e resultados de diferenciabilidade, que são conceitos locais, para podermos aproximar $f$ por uma aplicação diferenciável nos abertos $f^{-1}\left(W \backslash S^{1}\right)$ e $f^{-1}\left(W \backslash S^{2}\right)$.

Então existe um valor regular $y \in W \backslash S^{1}$. Pelo Teorema de Sard y possui uma vizinhança 2-Euclidiana $V \subset S^{2} \subset W$ tal que $V$ não contém valor crítico. Como o domínio $S^{2}$ é compacto e $y$ é valor regular, então $f^{-1}(y)$ é um número finito de pontos $\left\{x_{1}, \ldots, x_{t}\right\} \subset S^{2}$. Considere uma vizinhança $B_{1} \subset S^{2}$ de $x_{1}$ homeomorfa a um disco de dimensão 2 tal que $f\left(B_{1}\right) \subset V$ e $\partial B_{1} \cap f^{-1}(y)=\emptyset$. Por um resultado de [6] (pg. 300, Lema $5.3)$ tem-se que $f$ pode ser deformada por homotopia em uma aplicação $f^{1}: S^{2} \rightarrow W$ tal que $\left.f^{1}\right|_{\left(S^{2} \backslash B_{1}\right)}=\left.f\right|_{\left(S^{2} \backslash B_{1}\right)},\left(f^{1}\right)^{-1}(y) \cap B_{1}=\left\{x_{1}\right\}$ e $f^{1}\left(B_{1}\right) \subset V$.

Repetindo o processo acima para $x_{2}$ e depois sucessivamente até $x_{t}$, existirá $B_{1}, \ldots, B_{t}$ vizinhanças disjuntas, homeomorfas a disco de dimensão 2 , de respectivamente $x_{1}, \ldots, x_{t}$ tais que $f^{t}: S^{2} \rightarrow W$ satisfaz:

1) $\left.f^{t}\right|_{\left(S^{2} \backslash\left(B_{1} \cup \ldots \cup B_{t}\right)\right)}=\left.f\right|_{\left(S^{2} \backslash\left(B_{1} \cup \ldots \cup B_{t}\right)\right)}$,

2) $\left(f^{t}\right)^{-1}(y) \cap B_{i}=\left\{x_{i}\right\}$ e $f^{t}\left(B_{i}\right) \subset V$, para todo $i \in\{1, \ldots, t\}$,

3) $f^{t} \cong f$.

Podemos tomar um caminho simples $c: I \rightarrow W$, tal que $c$ conecta todos $B_{i}$ e $c(I) \subset$ 
$V \backslash \bigsqcup_{i=1}^{t} \stackrel{\circ}{B_{i}}$. Sem perda de generalidade podemos supor $V=\bigsqcup_{i=1}^{t} B_{i} \cup(c(I))$.

Considere a homotopia $H: W \times I \rightarrow W$ tal que $H(x, 0)=x$ e $H(x, 1)=r(x)$ onde $r: W \rightarrow W$ é uma aplicação tal que $r(V)=W \backslash S^{1}$ e $\left.r\right|_{S^{1}}=\left.\left(I d_{W}\right)\right|_{S^{1}}$, isto é, $r$ leva o aberto $V$ sobre $W \backslash S^{1}$, leva $\left(S^{2} \backslash V\right) \subset W$ em $\{P\}$ e o resto mantém a identidade, como no desenho abaixo
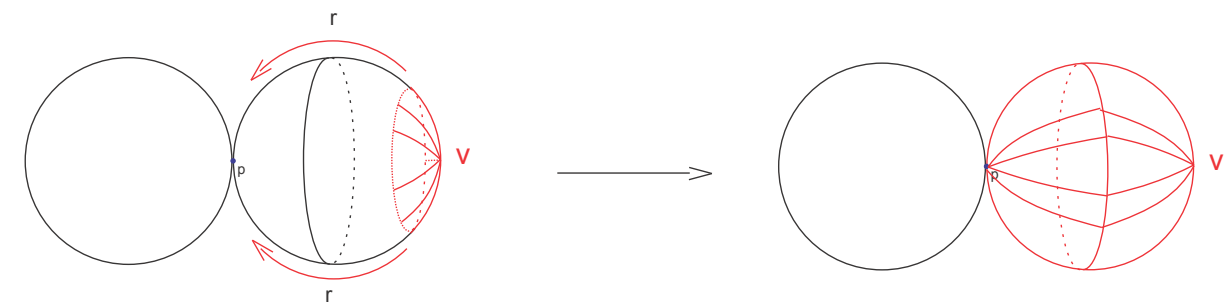

Figura 1.1: Retração $S^{2}$

Assim $f^{t} \cong f=H(., 0) \circ f \cong H(., 1) \circ f=r \circ f \cong r \circ f^{t}$. Portanto a menos de homotopia $f^{-1}\left(W \backslash S^{1}\right)$ é uma subvariedade de $S^{2}$ homeomorfa a $\bigsqcup_{t=1}^{n} B_{t}^{2}$, onde $n$ é finito e $B_{t}^{2}$ são discos abertos de dimensão $2, B_{t}^{2}$ é o interior de discos disjuntos $D_{t}^{2}$ tal que $\partial D_{t}^{2} \simeq S_{t}^{1}$ e tal que $f\left(\partial D_{t}^{2}\right)=\{P\}$ para $t \in\{1, \ldots, n\}$.

Além disso como $S^{2}$ e $W$ são compactos segue pelo corolário 4.2 de [6] que podemos considerar a aplicação $f$ tal que $\left.f\right|_{B_{t}^{2}}: B_{t}^{2} \rightarrow W \backslash S^{1}$ é homeomorfismo e se tomarmos $D_{t}^{2}=B_{t}^{2} \cup \partial D_{t}^{2}$ o grau de $f \mid D_{t}^{2}$ é \pm 1 .

O próximo lema juntará certos discos deste tipo.

Lema 1.1.2. Sejam uma aplicação $f: S^{2} \rightarrow W$ fortemente sobrejetora e seu levantamento $\widetilde{f}: S^{2} \rightarrow \widetilde{W}$, onde $\widetilde{W}$ é o recobrimento universal de $W$. Então existe uma aplicação $g: S^{2} \rightarrow W$ tal que $[g]=[f]$ e $\widetilde{g}^{-1}\left(S_{i}^{2} \backslash\{i\}\right)=B_{i}^{2}$ ou $\widetilde{g}^{-1}\left(S_{i}^{2} \backslash\{i\}\right)=\emptyset$, onde $\widetilde{g}: S^{2} \rightarrow \widetilde{W}$ é o levantamento de $g, B_{i}^{2}$ são discos abertos de dimensão dois e $i \in\{k, \ldots, k+n\}$.

Demonstração: Já que $S^{2}$ é compacto, $\widetilde{f}\left(S^{2}\right)$ também o é. Portanto existe um primeiro índice $k$ e um último índice $k+n$ tal que $S_{k}^{2}$ e $S_{k+n}^{2}$ são atingidas pela imagem de $\widetilde{f}$.

Pelo lema 1.1.1 podemos considerar que $f^{-1}\left(W \backslash S^{1}\right)$ é uma subvariedade de $S^{2}$ homeo- 
morfa a $\bigsqcup_{t=1}^{n} B_{t}^{2}$, onde $n$ é finito, $B_{t}^{2}$ são discos abertos de dimensão $2,\left.f\right|_{B_{t}^{2}}: B_{t}^{2} \rightarrow W \backslash S^{1}$ é homeomorfismo e $f\left(\partial B_{t}^{2}\right)=P$ para $t \in\{1, \ldots, n\}$. Portanto para cada $j \in\{k, \ldots, k+n\}$ $\tilde{f}^{-1}\left(S_{j}^{2} \backslash\{j\}\right)$ é uma subvariedade de $S^{2}$ homeomorfa a $\bigsqcup_{l=n_{j}}^{n_{j}+r} B_{l}^{2}$, onde $B_{l}^{2} \subset\left\{B_{1}^{2}, \ldots, B_{n}^{2}\right\}$, $\left.\widetilde{f}\right|_{B_{l}^{2}}: B_{l}^{2} \rightarrow S_{j}^{2} \backslash\{j\}$ é homeomorfismo com $\widetilde{f}\left(\partial B_{l}^{2}\right)=j$ para $l \in\left\{n_{j}, \ldots, n_{j}+r\right\}$ e $\left\{n_{j}, \ldots, n_{j}+r\right\} \subset\{1, \ldots, n\}$.

Fixemos $j \in\{k, \ldots, k+n\}$, juntaremos os discos $\bigsqcup_{l=n_{j}}^{n_{j}+r} B_{l}^{2}$ em um único disco.

Já que $S^{2} \backslash f^{-1}\left(W \backslash\left(S^{1}-\{P\}\right)\right)$ é conexo por caminhos, quaisquer dois pontos podem ser ligados por um caminhos em $S^{2} \backslash f^{-1}\left(W \backslash\left(S^{1}-\{P\}\right)\right)$. Portanto tomemos um caminho simples $c: I \rightarrow S^{2}$ tal que vale as seguintes propriedades:

i) $c(I) \subset S^{2} \backslash f^{-1}\left(W \backslash\left(S^{1}-\{P\}\right)\right)$.

ii) $c(0) \in \partial B_{n_{j}}^{2} \subset \bigsqcup_{l=n_{j}}^{n_{j}+r} \partial B_{l}^{2}, c\left(\frac{1}{r-1}\right) \in \partial B_{n_{j}+1}^{2} \subset \bigsqcup_{l=n_{j}}^{n_{j}+r} \partial B_{l}^{2}, \ldots, c\left(\frac{r-2}{r-1}\right) \partial B_{n_{j}+r-1}^{2} \subset$ $\bigsqcup_{l=n_{j}}^{n_{j}+r} \partial B_{l}^{2}$ e $c(1) \in \partial B_{n_{j}+r}^{2} \subset \bigsqcup_{l=n_{j}}^{n_{j}+r} \partial B_{l}^{2}$.

Por construção do caminho $c, \tilde{f} \circ c \in \mathbb{R} \subset \widetilde{W}$. Como $I$ é compacto segue que $\tilde{f} \circ c(I)$ também é compacto tal que $\tilde{f} \circ c(0)=\tilde{f} \circ c(1)=j$. Portanto, a menos de homotopia, podemos considerar que $\tilde{f} \circ c(I)=j$. Logo a menos de homotopia existe um único disco $D_{j} \subset S^{2}$ tal que $\tilde{f}\left(D_{j}\right) \subset S_{j}^{2}, \tilde{f}\left(\partial D_{j}\right)=j$ e o grau de $\left.\widetilde{f}\right|_{D_{j}}$ é a soma dos graus $\operatorname{deg} \tilde{f}\left|D_{1}^{2}+\ldots+\operatorname{deg} \widetilde{f}\right| D_{n_{j}}^{2}$. Se esta soma for zero então existe, a menos de homotopia, $\tilde{f}$ tal que $\tilde{f}\left(D_{j}\right)=j$.

Portanto existe uma homotopia $H_{j}: S^{2} \times I \rightarrow \widetilde{W}$ tal que $H_{j}(x, 0)=\widetilde{f}$ e $H_{j}(x, 1)=\widetilde{g_{j}}$ tal que $\widetilde{g}_{j}\left(D_{j}\right) \subset S_{j}^{2}, \widetilde{g}_{j}\left(\partial D_{j}\right)=j$ e o grau de $\left.\widetilde{g}_{j}\right|_{D_{j}}$ é a soma dos graus deg $\widetilde{g}_{j} \mid D_{1}^{2}+\ldots+$ $\operatorname{deg} \widetilde{g}_{j} \mid D_{n_{j}}^{2}$.

Repetindo este processo de junção para cada índice $i \in\{k, \ldots, k+n\}$ temos a existência de uma homotopia $H(x, 0)=\widetilde{f}$ e $H(x, 1)=\widetilde{g}$ tal que $\widetilde{g}^{-1}\left(S_{i}^{2} \backslash\{i\}\right)=B_{i}^{2}$ ou $\widetilde{g}^{-1}\left(S_{i}^{2} \backslash\{i\}\right)=\emptyset$ e $\widetilde{g}\left(\partial D_{i}\right)=i$, onde $D_{i}=B_{i}^{2} \cup \partial B_{i}^{2}$ e $i \in\{k, \ldots, k+n\}$.

Portanto a composição $p \circ H$ é uma homotopia tal que $p \circ H(x, 0)=p \circ \tilde{f}=f$ e $p \circ H(x, 1)=p \circ \widetilde{g}=g$ e $g$ é como queríamos. 
Lema 1.1.3. Seja uma aplicação $f: S^{2} \rightarrow W$ contínua e fortemente sobrejetora. Então, a menos de homotopia, $f^{-1}\left(W \backslash S^{2}\right)$ é uma subvariedade de $S^{2}$ homeomorfa a $\bigsqcup_{i=1}^{n} S_{i}^{1} \times(-\epsilon, \epsilon)$, onde $n$ é finito.

Demonstração: Assim como no lema anterior, podemos usar os conceitos e resultados de diferenciabilidade. O aberto $Z=W \backslash S^{2}$ de $W$ é homeomorfo a $\mathbb{R}$.

Consideremos os intervalos abertos $\widetilde{V} \subset \widetilde{U} \subset Z$ e um compacto $\widetilde{A} \subset \widetilde{V}$ tal que $\overline{\vec{V}} \subset \widetilde{U}$. Dados $f: S^{2} \rightarrow S^{1} \subset W$ contínua, tomemos os abertos de $S^{2}, U=f^{-1}(\widetilde{U}), V=f^{-1}(\widetilde{V})$ e o compacto $A=f^{-1}(\widetilde{A})$. Assim, vale que $A \subset V$ e $\bar{V} \subset U$. Seja $\left.f\right|_{U}: U \rightarrow \widetilde{U}$ a restrição de $f$. Pelo resultado 4.1 de [15] temos que existe uma aplicação $f_{1}: U \rightarrow \widetilde{U}$ tais que $f_{1}$ é $C^{\infty}$ numa vizinhança de $A, f_{1}=f$ para os pontos fora de $V$ e $f_{1}$ é homotópica a $f$. Dessa forma se definirmos $f_{1}(x)=f(x)$ para os pontos em $S^{2} \backslash U, f_{1}$ será contínua com as propriedades acima.

Portanto seja $x \in W \backslash S^{2}$ tal que $x$ é valor regular de $f_{1}$. Pelo teorema de Sard temos que existe uma vizinhança $V_{1}$ de $x$ tal que não contenha pontos críticos de $f_{1}$ em $V_{1}$. Considere a homotopia em $W, H: W \times I \rightarrow W$ tal que $H(x, 0)=x$ e $H(x, 1)=r(x)$ onde $r: W \rightarrow W$ é uma aplicação tal que $r\left(V_{1}\right)=W \backslash S^{2}$ e $\left.r\right|_{S^{2}}=\left.\left(I d_{W}\right)\right|_{S^{2}}$, ilustrada por,
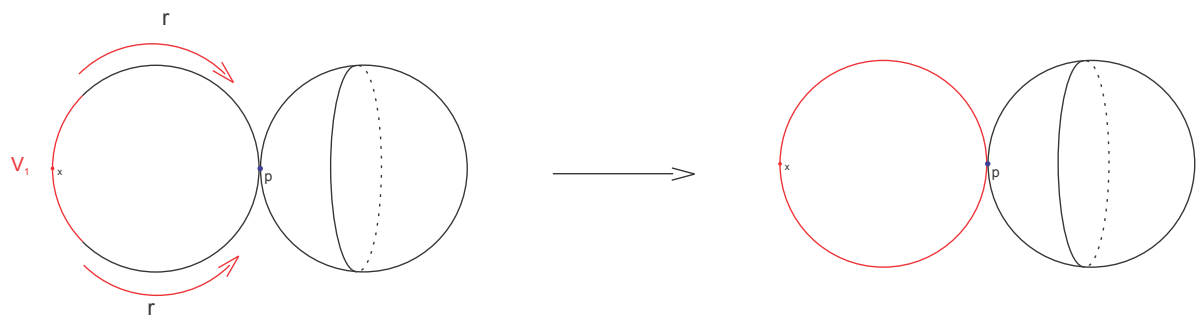

Assim $f_{1}=H_{0} \circ f_{1} \cong H_{1} \circ f_{1}=r \circ f_{1}$. Portanto podemos considerar que todos os pontos em $W \backslash S^{2}$ são valores regulares de $f_{1}$. Pelo fato de $x \in W \backslash S^{2}$ ser valor regular, $\left(f_{1}\right)^{-1}(x)$ é uma subvariedade de $S$ homeomorfa a uma união disjunta $\bigsqcup_{i=1}^{n} S_{i}^{1} \times(-\epsilon, \epsilon)$, onde $n$ é finito. Dessa forma $\left(f_{1}\right)^{-1}\left(W \backslash S^{2}\right)$ é uma subvariedade de $S$ homeomorfa a $\bigsqcup_{i=1}^{n} S_{i}^{1} \times(-\epsilon, \epsilon)$, onde $n$ é finito e $f_{1}$ é homotópica a $f$.

Sejam uma aplicação $f: S^{2} \rightarrow W$ e seu levantamento $\widetilde{f}: S^{2} \rightarrow \widetilde{W}$. Como $S^{2}$ é compacta, um número finito de $S_{i}^{2}$ esta contida na imagem de $\widetilde{f}$. Suponha que $S_{k}^{2}$ é a 
esfera que esta contida na imagem tal que $k$ seja o maior inteiro e que a imagem de $\tilde{f}$ atinja o intervalo $[k, k+n]$ e $\operatorname{Im}(\widetilde{f}) \cap(k+n,+\infty)=\emptyset$.

Como a última esfera atingida pela $\widetilde{f}$ é $S_{k}^{2}$, então existe uma aplicação $r: \widetilde{W} \rightarrow \widetilde{W}$ que retrai todo intervalo $[k, k+n]$ no ponto $k$. A imagem da composição $r \circ \tilde{f}$ não atinge mais todo intervalo $[k, k+n]$, mas somente o ponto $k$. Além disso, esta composição é homotópica a $\widetilde{f}$ e portanto $r \circ \widetilde{f} \circ p$ é homotópica a $f$.

Pelo Lema 1.1.3 existe um cilindro $C \cong S^{1} \times[-\epsilon, \epsilon] \subset S^{2}$ tal que $\widetilde{f}(C)=[k, k+1] \subset \widetilde{W}$ e $f(C)=S^{1}$. Quando fazemos a homotopia acima, significa que mudamos a aplicação $f$ tal que a imagem de $C$ seja o ponto $P \in S^{1}$.

Pelo lema 1.1.2 $\tilde{f}^{-1}\left(S_{k+n}^{2} \backslash\{k+n\}\right)=B_{k+n}^{2}$ e se a esfera $S_{k+n}^{2}$ é coberta por $f$ com grau igual a zero, então, por propriedade de grau, a aplicação $f$ é homotópica a uma aplicação que é constante em $B_{k+n}^{2}$. Logo repete-se o processo acima para $[k+n-1, k+n]$.

Podemos repetir este processo finitas vezes. Logo sem perda de generalidade, tomamos $k+n$ como sendo o menor inteiro tal que $S_{k+n}^{2}$ esta contida na imagem de $\tilde{f}$ com grau diferente de zero.

Portanto, a menos de homotopia, a imagem $\tilde{f}$ não atinge o subconjunto $(k+n,+\infty) \cup$ $(-\infty, k)$, onde $k+n$ é o maior inteiro tal que $S_{k+n}^{2}$ pertence a imagem de $\widetilde{f}, k$ é o menor inteiro tal que $S_{k}^{2}$ pertence a imagem de $\tilde{f}$ e as esferas $S_{k+n}^{2} S_{k}^{2}$ são recobertas com graus diferentes de zero.

Quando foi feita a interpretação geométrica do polinômio associado $[f]$, vimos que podemos multiplicar por inversíveis $t^{j}, \forall j \in \mathbb{Z}^{*}$, isto é, a menos de escolha de ponto base do espaço $\widetilde{W}, k=0$.

Daqui por diante $k=0$.

Portanto depois de algumas homotopias terminamos com uma aplicação $\tilde{f}: S^{2} \rightarrow \widetilde{W}$ com boas propriedades, mas ainda $p \circ \tilde{f}$ não é uma aplicação especial.

Pelo Lema 1.1.3 a imagem inversa de cada intervalo $(j, j+1) \subset \widetilde{W}$, eventualmente, é uma união finita de cilindros, mas para uma aplicação especial a imagem inversa de cada intervalo $(j, j+1) \subset \widetilde{W}$ é vazio ou um único cilindro. Para eliminar os, possíveis, cilindros extras será utilizado teoria de grafos, através da fatoração de Stein que gera um grafo (também conhecido como grafo de Reeb ou grafo de Lyapunov). 
Existe uma retração $\phi: \widetilde{W} \rightarrow \mathbb{R}$ que manda cada esfera $S_{i}^{2}$ a $\{i\}$ e é a identidade em $\mathbb{R}$, isto é, $\left.\phi\right|_{\mathbb{R}}$ é a identidade e $\phi\left(S_{i}^{2}\right)=i$.

Podemos compor a aplicação $\tilde{f}$ com $\phi$ para obter um tipo de "aplicação de Morse" $\bar{f}$ : $S^{2} \rightarrow \mathbb{R}$. Esta aplicação tem imagem o intervalo $[0, n]$ e para todo inteiro $0 \leq i \leq n$ temos $\bar{f}^{-1}(i)$ é uma coleção não vazia de 2 -variedades com bordo de genus zero e $\bar{f}^{-1}((i, i+1))$ é uma coleção não vazia de cilindros $\left(\simeq S^{1} \times(-\epsilon, \epsilon)\right)$.

Consideramos a fatoração Stein de $\bar{f}$, isto é, definimos uma relação de equivalência no domínio $S^{2}$ por $x_{1} \sim x_{2}$ se $f\left(x_{1}\right)=f\left(x_{2}\right)$ e os pontos $x_{1}$ e $x_{2}$ pertencem a mesma componente conexa de $f^{-1}\left(x_{1}\right)$. O espaço quociente $S^{2} / \sim$ é um grafo denotado por $\Gamma_{\bar{f}}$ e neste caso o grafo é uma árvore. Definimos a aplicação $\overline{f_{s}}: \Gamma_{\bar{f}} \rightarrow \mathbb{R}$ por $\overline{f_{s}}(\bar{x})=\bar{f}(x)$, onde $\bar{x}$ é a classe de $x \in S^{2}$ e temos o seguinte diagrama comutativo

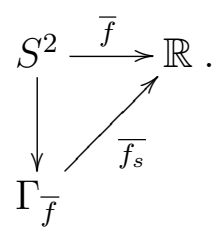

A aplicação $\bar{f}$ pode ser vista como uma função de Morse com "regiões planas". Neste caso o domínio da fatoração de Stein $\Gamma_{\bar{f}}$ é uma árvore que tem seus vértices enviados por $\overline{f_{s}}$ nos inteiro de $\mathbb{R}$ e suas arestas correspondem aos cilindros que são projetados nos intervalos aberto $(i, i+1)$. Chamaremos $\bar{f}_{s}: \Gamma_{\bar{f}} \rightarrow \mathbb{R}$ a aplicação induzida de $f$ na fatoração de Stein.

Pelo Lema 1.1.2, há um único disco aberto $B_{i}^{2}$ tal que $\widetilde{f}\left(B_{i}^{2}\right)=S_{i}^{2} \backslash\{i\}, \widetilde{f}\left(\partial B_{i}^{2}\right)=i$ e $\left.\widetilde{f}\right|_{\overline{B_{i}^{2}}}:\left(\overline{B_{i}^{2}}, \partial B_{i}^{2}\right) \rightarrow\left(S_{i}^{2}, i\right)$ recobre a esfera $S_{i}^{2}$ com grau $a_{i}$. Assim, em cada nível $i$ tal que $a_{i} \neq 0$ destacamos o vértice de $\Gamma_{\bar{f}}$ que corresponde à superfície que contém o $\partial B_{i}^{2}$ e "pintamos de vermelho", para distingui-los dos demais.

Note que as "regiões planas"são as superfícies com bordo que dão origem aos vértices do grafo, eles são mandados por $\bar{f}$ nos inteiros $\{0, \ldots, n\}$.

Nós definimos uma ordem parcial no grafo, usando a aplicação $\bar{f}$ e a ordem de $[0, n] \subset \mathbb{R}$. Assim podemos imaginar $\bar{f}$ como a função altura definida de $S^{2}$ no grafo $\Gamma_{\bar{f}}$. Abreviaremos $\Gamma_{\bar{f}}$ por $\Gamma$.

Será feita modificações, por homotopias, em $\bar{f}$, mas ainda manteremos o mesmo nome 
para esta nova aplicação e para o novo grafo também manteremos a notação $\Gamma$.

A partir de agora vamos estudar as mudanças por homotopias de $f$ a partir do que ocorre com o grafo $\Gamma$ e na aplicação $\overline{f_{s}}$.

Começaremos um processo de mudança por homotopias da aplicação $\bar{f}$ sem alterações $\operatorname{nos}$ discos $E_{i}$ (os discos que a aplicação $\tilde{f}$ manda no interior das esferas $S_{i}^{2}$ onde $a_{i} \neq 0$ ). Devemos fazer isto de tal forma que o grafo se torne o grafo de uma aplicação especial o qual é linear e estritamente crescente com $(n+1)$ vértices e $n$ arestas.

Passo 1 Se no nível $n$ tiver mais vértices do que o "vértice vermelho", podemos eliminar os outros vértices por uma homotopia de $\widetilde{f}$ tal que as superfícies correspondentes a estes vértices e os cilindros atingidos são movidos para o nível $(n-1)$.

Note que neste caso o número de vértices nos níveis $(n-1)$ diminui, uma vez que algumas superfícies desse nível estão ligados pelos cilindros.

Passo 2 Podemos fazer o mesmo com o nível 0 e 1.

Passo 3 Usando o mesmo processo, podemos eliminar "vértices de máximos locais não vermelhos"e "vértices de mínimos locais não vermelhos".

Neste ponto, temos uma aplicação $\tilde{f}$ tal que o grafo $\Gamma$ de $\bar{f}$ tem apenas um vértice maximal (vermelho) e apenas um vértice minimal (vermelho).

Considere um subgrafo $\Delta \subset \Gamma$ ligando o vértice vermelho do nível 0 ao vértice vermelho do nível $n$ tal que $\Delta$ é um caminho simples, ou seja, $\Delta$ é um caminho sem auto interseção.

Passo 4 Neste momento, temos uma árvore de modo que o (único) vértice minimal no nível 0, o (único) vértice máximo no nível $n$ são os vermelhos e com possíveis arestas livres com vértices extremos vermelhos.

$\Delta$ é um grafo linear (não necessariamente crescente) que liga o vértice vermelho de nível 0 ao vértice vermelho do nível $n$, se este subgrafo for estritamente crescente e contém todos os vértices vermelhos, então o processo acabou uma vez que este gráfico é um 
grafo de uma aplicação especial (a menos de mais algumas homotopias simples, onde as superfícies planares tornam-se objetos 1-dimensionais e as arestas livres são eliminadas por homotopias triviais).

Lema 1.1.4. Sejam $f: S^{2} \rightarrow W$ uma aplicação contínua e fortemente sobrejetora, $\tilde{f}: S^{2} \rightarrow \widetilde{W}$ e $\bar{f}=\phi \circ \widetilde{f}: S^{2} \rightarrow \mathbb{R}$ como antes. Podemos mudar $\tilde{f}$, por homotopias, tal que o grafo $\Gamma$ é linear e estritamente crescente.

Demonstração: Se o gráfico $\Gamma$ é estritamente crescente (ou estritamente decrescente) acabou. Então suponha que o gráfico contém três vértices $A, B$ e $C$, tal que $A, C$ estão no nível $i$ e $B$ está no nível $i+1$, como na ilustração abaixo:

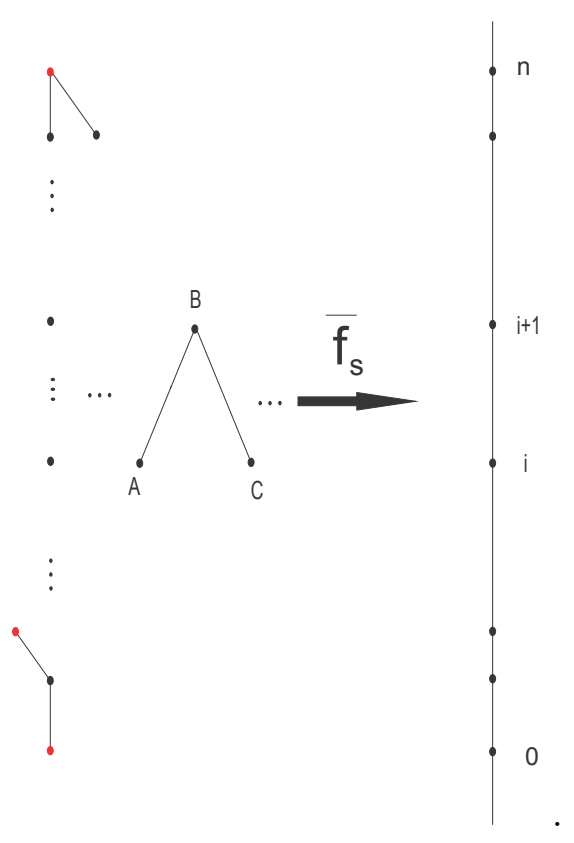

Os vértices $A$ e $C$ correspondem à componentes conexas por caminhos de $\widetilde{f}^{-1}(i), C_{i_{A}} \mathrm{e}$ $C_{i_{C}}$, respectivamente; o vértice $B$ corresponde à componente conexa por caminhos $C_{(i+1)_{B}}$ de $\widetilde{f}^{-1}(i+1)$, e as arestas $\overline{A B}$ e $\overline{B C}$ correspondem à cilindros de $\widetilde{f}^{-1}((i, i+1))$.

Consideramos três pontos $x_{1} \in C_{i_{A}}, x_{2} \in C_{(i+1)_{B}}$ e $x_{3} \in C_{i_{C}}$. Tomamos um caminho simples $c: I \rightarrow \tilde{f}^{-1}([i, i+1])$ conectando $x_{1}$ a $x_{3}$, passando por $x_{2}$ tal que não atinge nenhum outro cilindro, exceto aqueles que correspondem a $A B$ e $B C$.

Tomamos $V \subset S^{2} \backslash \tilde{f}^{-1}\left(W \backslash S^{1}\right)$ uma vizinhança tubular de $c$. Desde que $\tilde{f}(c(I)) \subset \mathbb{R}$ e os pontos finais são mandados em $i$, a menos de homotopia, $f(c(I))=P$. Utilizamos 
esta homotopia para modificar $f$ em $V$ de tal modo que o novo grafo associado a nova $\bar{f}$ seja como se segue:

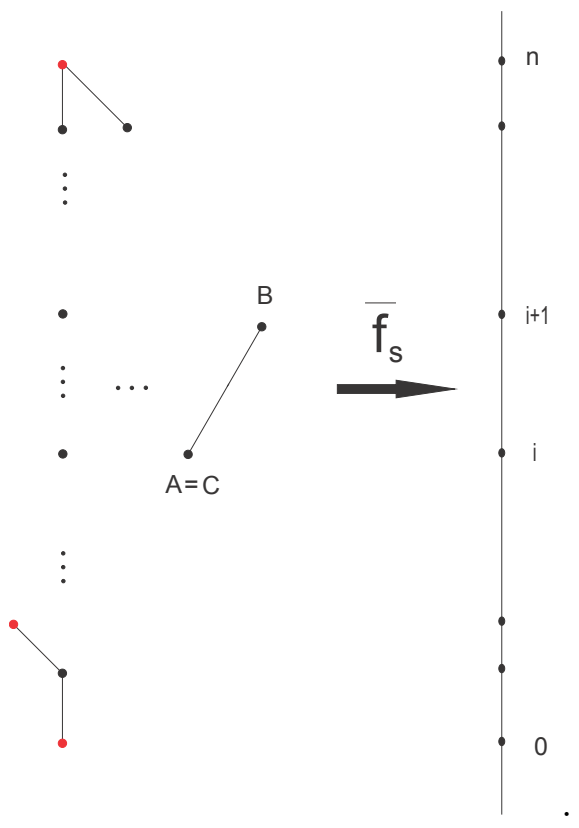

Repetindo este processo para as outras partes de $\Gamma$ similares a parte considerada acima entre os níveis $i$ e $i+1$.

Terminamos com um grafo linear estritamente crescente o qual é associado a uma aplicação especial.

Teorema 1.1.1. Dada uma aplicação $f: S^{2} \rightarrow W$ e sua classe de homotopia $[f]$. Então existe $g \in[f]$ tal que g é especial.

Demonstração: Considere a aplicação $f: S^{2} \rightarrow W$. Pelos lemas 1.1.1 e 1.1.3 existe uma aplicação homotópica a $f$, que manteremos o nome $f$, tal que $f^{-1}\left(W \backslash S^{1}\right)$ é uma união finita de bolas cujos bordos são enviados por $f$ em $\{P\}, f^{-1}\left(W \backslash S^{2}\right)$ é uma união finita de cilindros $S^{1} \times(-\epsilon, \epsilon)$, os conjuntos $\left(S^{1} \times\{-\epsilon\}\right) \cup\left(S^{1} \times\{\epsilon\}\right)$ são enviados por $f$ em $P$ e $f^{-1}(P)$ é uma coleção de superfícies compactas, conexas e com bordo de gênus zero.

Considere $\widetilde{f}: S^{2} \rightarrow \widetilde{W}$ o levantamento de $f$.

A imagem de $\tilde{f}$ é um conjunto compacto e conexo, assim a imagem é apenas um segmento $[a, b] \subset \mathbb{R}$, unido com um número finito de esferas. 
Observa-se que as esferas que podem ser atingidas por $\tilde{f}$ são $S_{n}^{2}, S_{n+1}^{2}, \ldots, S_{n+k}^{2}$ tais que $\{n, n+1, \ldots, n+k\} \subset[a, b]$ as quais (não necessariamente todas elas) são as imagens dos discos $D_{i}^{2}$ pelo Lema 1.1.1.

Para cada uma dessas esferas $S_{j}^{2}$ coletamos os discos $D_{i}^{2}$ que são enviados por $\tilde{f}$ nesta esfera. Se o número deles é maior do que um, então conectamos estes discos por um caminho simples desviando dos outros discos de tal modo que a união dos discos e uma vizinhança suficientemente pequena do caminho será um único disco que contém todos os discos que atingem esta esfera.

Pelo Lema 1.1.2 o aplicação $\tilde{f}$ pode ser deformada mais uma vez ao longo do disco e da vizinhança do caminho (mais uma vez, continuaremos chamando a nova aplicação de $\tilde{f}$ ) de forma que a imagem de uma pequena vizinhança do caminho tenha imagem $\{j\} \subset \mathbb{Z} \subset \mathbb{R} \subset \widetilde{W}$ (note que $p \circ \widetilde{f}: S^{2} \rightarrow W$ envia este caminho em $P$ ). Após isso, ajustamos $\tilde{f}$ por outra homotopia tal que em um único disco a aplicação $\widetilde{f}$ tem grau igual a soma algébrica dos graus dos discos correspondentes.

Se o grau é igual a zero, então a aplicação restrita ao disco é homotópica a aplicação constante, portanto, mudaremos homotopicamente a aplicação uma vez mais de modo que a imagem não atinge o interior da esfera, ou seja é constante e igual a $P$.

Depois de repetir esse processo para as outras famílias de discos que atingem a mesma esfera, temos uma aplicação $\widetilde{f}: S^{2} \rightarrow \widetilde{W}$ tal que para uma coleção de discos $E_{k}^{2},\left.\widetilde{f}\right|_{E_{k}^{2}}$ : $\left(E_{k}^{2}, \partial E_{k}^{2}\right) \rightarrow\left(S_{k}^{2}, k\right)$ tem grau $a_{k} \neq 0$.

Chamamos $k^{+}$o maior índice de tal forma que $a_{k^{+}} \neq 0, k^{-}$o menor índice tal que $a_{k^{-}} \neq 0$ e, por conveniência, escolhemos o levantamento de $f$ a $\widetilde{W}$ tal que $k^{-}=0$ e $k^{+}=n$.

Então, para $i>n$ ou $i<0, S_{i}^{2} \backslash\{i\}$ não é atingido por $\widetilde{f}$.

Podemos ter algum $0<i<n$ tal que $a_{i}=0$. Neste caso, mudamos a aplicação por uma homotopia e temos que o interior da esfera $S_{i}^{2}$ não é atingido por $\widetilde{f}$.

Note que temos uma aplicação $\widetilde{f}$ tal que a parte da imagem de $\widetilde{f}$ que atinge $\widetilde{W}$ acima de $n$ esta dentro do segmento $[n, b] \subset \mathbb{R}$, o qual é contrátil (o mesmo para a imagem de $\tilde{f}$ abaixo 0). Assim, podemos modificar $\tilde{f}$ tal que a imagem de $\tilde{f}$ é o conjunto $[0, n] \cup\left(\cup S_{i}^{2}\right)$, onde $a_{i} \neq 0$ e o conjunto de índices $i$ é um subconjunto de $\{0, \ldots, n\}$. 
Observamos que a imagem inversa de cada $i \in \mathbb{Z}$ é uma coleção de superfícies com bordo de genus zero (possíveis círculos ou "wedges"de círculos) e para cada intervalo $(j, j+1)$ a imagem inversa é uma coleção não vazia de cilindros.

Consideramos a fatoração de Stein (ou grafo de Lyapunov) de $\bar{f}: S^{2} \rightarrow \mathbb{R}$, isto é, definimos uma relação de equivalência no domínio $S^{2}$ por $x_{1} \sim x_{2}$ se $f\left(x_{1}\right)=f\left(x_{2}\right)$ e os pontos $x_{1}$ e $x_{2}$ pertencem a mesma componente conexa de $f^{-1}\left(x_{1}\right)$. O espaço quociente $S^{2} / \sim$ é um grafo, denotado por $\Gamma$, que neste caso é uma árvore. Definimos a aplicação $\overline{f_{s}}: \Gamma_{\bar{f}} \rightarrow \mathbb{R}$ por $\overline{f_{s}}(\bar{x})=\bar{f}(x)$, onde $\bar{x}$ é a classe de $x \in S^{2}$ e temos o seguinte diagrama comutativo

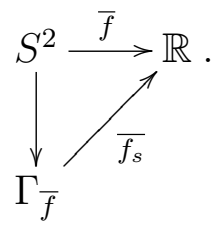

Em $\Gamma$ é dado uma ordem parcial induzida por $\bar{f}$ e pela ordem de $\mathbb{R}$.

Os vértices que correspondem às superfícies que contém os $\partial D_{i}^{2}$ com $a_{i} \neq 0$ é destacado em vermelho.

Pelos Passos 1, 2, 3, 4 e o Lema 1.1.4 temos uma abordagem para mudar, homotopicamente, a aplicação $\bar{f}$, sem alterá-la no interior de $D_{i}$. Estas mudanças são parametrizadas pela fatoração de Stein que por fim resulta em um grafo linear e estritamente crescente que liga os vértices extremos e passando por cada vértice vermelho de $\Gamma$, e isto é um grafo que corresponde a uma aplicação especial, é fácil ver que $\widetilde{f}$ é especial.

\subsection{Representação das classes de homotopia de aplica- ções de $T^{2}$ em $W$}

Também neste caso a cada classe de homotopia de uma aplicação $f: T^{2} \rightarrow W$ será associado um polinômio. A partir deste polinômio será construída uma aplicação que terá boas propriedade e será chamada de especial. Em seguida, será provado que em cada classe de homotopia $[f]$ existe uma aplicação especial que pode ser dada a partir do 
polinômio associado a classe $[f]$.

Eventualmente, nesta seção e nas próximas seções, será considerado o homomorfismo induzido por $f: S \rightarrow W_{n}$ em grupo fundamental, para isso é necessário fixar pontos bases de $S$ e $W_{n}$ e considerar $f$ baseada. Para simplificar não é denotado os pontos bases dos espaços.

\section{Polinômio associado a uma aplicação $f: T^{2} \rightarrow W$}

Sejam uma aplicação $f: T^{2} \rightarrow W$, o grupo fundamental $\pi_{1}\left(T^{2}\right) \cong \mathbb{Z} \oplus \mathbb{Z}$ e $f_{\pi}$ : $\pi_{1}\left(T^{2}\right) \rightarrow \pi_{1}(W)$ a induzida de $f$ no grupo fundamental. Vamos supor que $l \in \mathbb{N}$ é tal que $<l>=f_{\pi}\left(\pi_{1}\left(T^{2}\right)\right) \operatorname{com} f_{\pi}(1,0)=a, f_{\pi}(0,1)=b$ e $m d c\{a, b\}=l$. Como $m d c\{a, b\}=l$, existem $x_{0}, y_{0} \in \mathbb{Z}$ tais que $x_{0} a+y_{0} b=l$. A base considerada em $\pi_{1}\left(T^{2}\right)=\mathbb{Z} \oplus \mathbb{Z}$ é a canônica $\{(1,0),(0,1)\}$ tal que geometricamente é representada pelos seguintes laços:

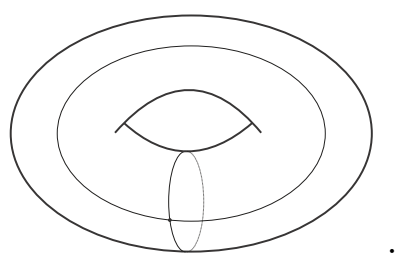

Se escolhermos o conjunto de pares $B=\left\{\left(x_{0}, y_{0}\right),\left(\frac{-b}{l}, \frac{a}{l}\right)\right\}$ como base para o $\pi_{1}\left(T^{2}\right)$, então temos $f_{\pi}\left(x_{0}, y_{0}\right)=x_{0} a+y_{0} b=l$ e $f_{\pi}\left(\frac{-b}{l}, \frac{a}{l}\right)=\frac{-b a}{l}+\frac{a b}{l}=0$, isto significa que se considerarmos o toro como o quociente de $I \times I$ identificando os bordos opostos, então a mudança para a base $B$ é apenas a troca de $I \times I$ por um losango e identifica-se os bordos opostos, onde os lados horizontais seriam o laço representado por $\left(x_{0}, y_{0}\right)$ e os lados verticais seriam o laço representado por $\left(\frac{-b}{l}, \frac{a}{l}\right)$.

Observe que esta mudança significa um homeomorfismo no domínio de $f$.

Dessa maneira dada uma aplicação como acima, sempre podemos escolher como modelo uma aplicação que leva o lado horizontal do quadrado $I \times I$, que é o círculo longitudinal no toro que corresponde ao gerador $\alpha=(1,0) \in \pi_{1}\left(T^{2}\right)$, na $S^{1}$ e outro lado do quadrado que corresponde ao outro círculo gerador $\beta=(0,1) \in \pi_{1}\left(T^{2}\right)$ no ponto $P$, como na ilustração 


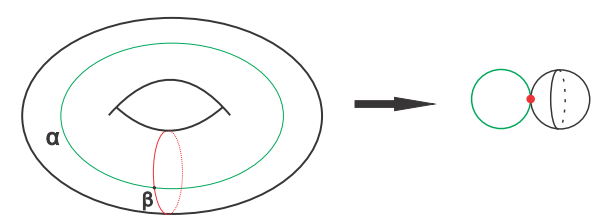

Figura 1.2:

A partir da aplicação $f: T^{2} \rightarrow W$ construímos uma aplicação $f_{S^{2}}: S^{2} \rightarrow W$ da seguinte forma:

- Identificamos o conjunto $T^{2} \backslash \beta$ com $S^{2} \backslash\left(D^{1} \cup D^{2}\right)$, onde $D^{i}$ são discos de dimensão 2 tal que $D^{1} \cap D^{2}=\emptyset$, como na figura abaixo

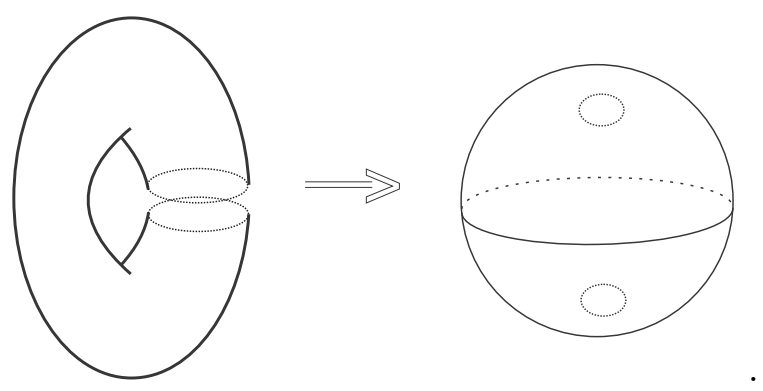

- Definimos a aplicação $f_{S^{2}}$ no conjunto $D^{1} \cup D^{2}$ como sendo a aplicação constante em $P$, isto é, $f_{S^{2}}\left(D^{1} \cup D^{2}\right)=P \in W$.

- Para todo $x \in S^{2} \backslash\left(D^{1} \cup D^{2}\right)$ definimos $f_{S^{2}}(x):=f(x)$.

Definição 1.2.1. Sejam uma aplicação $f: T^{2} \rightarrow W$ e o polinômio $p_{\left[f_{S^{2}}\right]}(t)$. Então $p_{[f]}(t)=p_{\left[f_{S^{2}}\right]}(t)$ é o polinômio associado a $[f]$, como na seção anterior.

\section{Representação quando $f_{\pi}=0$}

Consideremos as classes de homotopia $[f] \in\left[T^{2}, W\right]$ tais que $f_{\pi}: \pi_{1}\left(T^{2}\right) \rightarrow \pi_{1}(W)$ é o homomorfismo nulo. Neste caso podem ocorrer as seguintes situações:

- Se, a menos de homotopia, $f\left(T^{2}\right) \subset W \backslash S^{1}$ ou $f\left(T^{2}\right) \subset W \backslash S^{2}$, então $f$ é homotópica a uma função constante.

- Se, a menos de homotopia, $f\left(T^{2}\right)=S^{2} \subset W$, então usando as técnicas das demonstrações dos Lemas 1.1.2 1.1.1, a menos de homotopia temos $f^{-1}(P)=S^{1} \vee S^{1}$ e $f^{-1}\left(W \backslash S^{1}\right)=D^{2}$ 
tal que $\partial D^{2}=S^{1} \vee S^{1}$ e $\left.f\right|_{\left(D^{2}, \partial D^{2}\right)}$ recobre $S^{2}$ com grau diferente de zero, onde $D^{2}$ é um disco aberto. Quando o toro é pensado como o quadrado $I \times I$ identificando os bordos paralelos, a menos de homotopia, o conjunto $f^{-1}(P)$ são os bordos paralelos e $f^{-1}\left(W \backslash S^{1}\right)$ é o interior do quadrado $I \times I$.

- Se, a menos de homotopia, $f\left(T^{2}\right)=S^{1} \subset W$, então, desde que $f_{\pi}=0, f$ é homotópica a uma aplicação constante.

- Finalmente o caso em que $f$ é fortemente sobrejetora. Neste caso $f$ pode ser fatorada por $S^{2}$, isto é, existe uma aplicação $q: S^{2} \rightarrow W$ tal que o diagrama

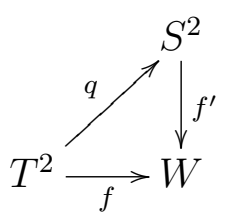

comuta.

Já vimos que $q$ pode ser representada utilizando as técnicas das demonstrações dos Lemas 1.1.1 e 1.1.2 e a aplicação $f^{\prime}$ é representada pelo seu polinômio, isto é, a menos de homotopia $f^{\prime}$ é especial.

Logo, a menos de homotopia, $f$ é tal que $q\left(S^{1} \vee S^{1}\right)$ é um único ponto $N \in S^{2}$ para algum $N \in S^{2},\left.q\right|_{\left(T^{2} \backslash S^{1} \vee S^{1}\right)}$ é uma bijeção com $S^{2} \backslash N$ e $f^{\prime}$ é uma aplicação especial representada como na seção 1.1 pelo seu polinômio $p_{\left[f^{\prime}\right]}(t)=p_{[f]}(t)$.

\section{Construção de $f: T^{2} \rightarrow W$ a partir de $q(t)$ e $l \in \mathbb{N}$}

Sejam um polinômio $q(t)=a_{0}+\ldots+a^{n} t^{n}$ e um número $l \in \mathbb{N}$. Considere a aplicação $f$ de $T^{2}$ em $W$ obtida da figura

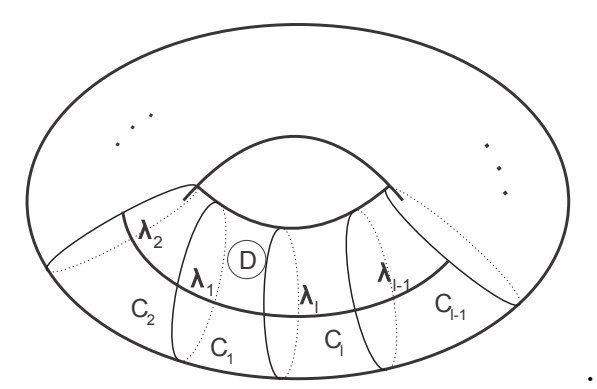


onde $f(\partial D)=P,\left.f\right|_{(D, \partial D)}:(D, \partial D) \rightarrow W$ é construída a partir do polinômio $q(t)$ como na seção 1.1 (como aplicação de $S^{2}$ em $W$ ) e a imagem de cada cilindro $C_{i}$ é $S^{1} \subset W$ tal que cada um dos caminhos $\lambda_{i}$ é levado pela $f$ em $S^{1} \subset W$ no sentido anti-horário e $i \in\{1, \ldots, l\}$.

Quando $l=0$, então $f\left(T^{2} \backslash \stackrel{\circ}{D}\right)=P,\left.f\right|_{(D, \partial D)}:(D, \partial D) \rightarrow W$ é construída a partir do polinômio $q(t)$ como na seção 1.1. Neste caso $f$ é equivalente (por homotopia) a aplicação tal que $f\left(S^{1} \vee S^{1}(=\alpha \vee \beta)\right)=P$ e $f\left(T^{2} \backslash S^{1} \vee S^{1}\right)$ é construída a partir do polinômio $q(t)$.

Portanto por construção $p_{[f]}(t)=q(t)$ e desde que $\pi_{1}\left(T^{2}\right) \cong \mathbb{Z} \oplus \mathbb{Z}=\langle\alpha>\oplus<\beta>$, então $f_{\pi}\left(\pi_{1}\left(T^{2}\right)\right) \simeq l \mathbb{Z}$, onde $f_{\pi}(\alpha)=l$ e $f_{\pi}(\beta)=0$.

Definição 1.2.2. Uma aplicação $f: T^{2} \rightarrow W$ será chamada especial se ela pode ser construída como acima, isto é, a partir do polinômio $p_{[f]}(t)$ e de $l$, onde $l \in \mathbb{N}$ é um gerador de $f_{\pi}\left(\pi_{1}\left(T^{2}\right)\right)$ ou $l=0$.

\section{Representação quando $f_{\pi} \neq 0$}

Quando uma aplicação $f: T^{2} \rightarrow W$ é tal que $f_{\pi} \neq 0$, temos um subgrupo $\operatorname{Im}\left(f_{\pi}\right) \subset \mathbb{Z}$. Logo existe $l \in \mathbb{N}$ tal que $\operatorname{Im}\left(f_{\pi}\right) \simeq \mathbb{Z}<l>\subset \mathbb{Z} \simeq \pi_{1}(W)$.

Suponha que, a menos de homotopia, $f\left(T^{2}\right)=S^{1} \subset W$. Neste caso temos a aplicação $f: T^{2} \rightarrow S^{1}$ e podemos fazer uma aproximação de $f$, isto é, a menos de homotopia $f$ é $C^{\infty}$. Com isso construímos o seguinte diagrama comutativo

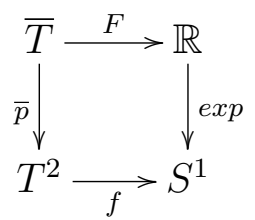

onde $\exp : \mathbb{R} \rightarrow S^{1}$ é aplicação exponencial usual, $\bar{T}$ é o recobrimento cíclico infinito de $T^{2}$ (mais adiante apresentaremos mais informações sobre $\bar{T}$ ). Portanto $\bar{p}$ é um difeomorfismo local.

Dado um aberto $U \subset S^{1}$ e o aberto $V=f^{-1}(U)$ tal que $\left.f\right|_{V}$ é $C^{\infty}$. Como exp e $\bar{p}$ são difeomorfismos locais, tomemos um respectivo aberto $U^{\prime} \subset \mathbb{R}$ e um respectivo aberto 
$V^{\prime} \subset \bar{T}$. Usamos resultados de Teoria de Morse para tomar uma aplicação $G \cong F$ tal que $G$ é uma aplicação de Morse. Temos então que podemos aproximar, usando o diagrama comutativo acima, a aplicação $F$ por uma aplicação de Morse, obtendo assim $f: T^{2} \rightarrow S^{1}$ uma aplicação de Morse circular.

Portanto para cada ponto $x \in S^{1}$ a imagem inversa $f^{-1}(x)$ pode ser uma união de $l$ cópias de $S^{1}$, isto é, $\bigsqcup_{i=1}^{l} S_{i}^{1}$. Pela teoria de Morse temos que $l$ é o mínimo de cópias de $S^{1}$ pertencente a imagem inversa $f^{-1}(x)$. Podendo, eventualmente, ter mais do que $l$.

Esta teoria que estuda aplicações de variedades fechadas $M$ em $S^{1}$ é chamada "Circle Valued Morse Theory"e uma referência sobre este assunto é [17].

De agora em diante estaremos considerando aplicações $f: T^{2} \rightarrow W$ fortemente sobrejetora.

Primeiramente vamos supor que $l=1$ e da mesma forma como foi feito no início desta seção, podemos supor, sem perda de generalidade, que $f_{\pi}(1,0)=1$ e $f_{\pi}(0,1)=0$, onde $\alpha=(0,1)$ e $\beta=(0,1)$ são como na ilustração 1.2 ,

Consideremos $\bar{T}$ como na ilustração seguinte,

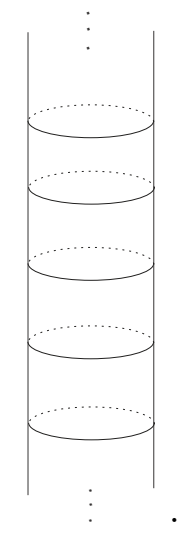

O espaço $\bar{T}$ é um espaço de recobrimento de $T^{2}$ relativo ao $k e r\left(f_{\pi}\right)$, isto é, um recobrimento cíclico infinito de $T^{2}$ tal que o diagrama

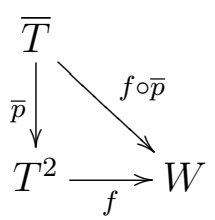

é comutativo. 
Por construção do recobrimento $\bar{T}$, a imagem $(f \circ \bar{p})_{\pi}\left(\pi_{1}(\bar{T})\right)$ é nula. Portanto a aplicação $f \circ \bar{p}$ se levanta ao recobrimento universal $\widetilde{W}$, isto é, o diagrama

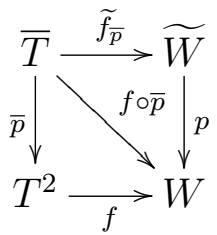

é comutativo.

Como este diagrama comuta, aplicações $\widetilde{f}_{\bar{p}}$ e $\widetilde{g}_{\bar{p}}$ homotópicas induzem aplicações $f$ e $g$ homotópicas.

Portanto faremos homotopias na aplicação $\widetilde{f}_{\bar{p}}$ para encontrar $\widetilde{g}_{\bar{p}} \simeq \widetilde{f}_{\bar{p}}$ tal que $\widetilde{g}_{\bar{p}}$ irá induzir uma aplicação $g \simeq f$, onde $g$ é especial.

O cilindro $\bar{T}$ é um recobrimento com $\mathbb{Z}$-folhas de $T^{2}$. Isto significa que basta conhecer como são as aplicações $\bar{p}$ e $\tilde{f}_{\bar{p}}$ em uma região fundamental do recobrimento (que é um cilindro fechado), pois as aplicações restritas em cada cilindro se repetem nas outras regiões.

Fixaremos uma destas folhas e chamaremos de $C$ a região destacada (cilindro fechado com bordos $\alpha_{0} \sqcup \alpha_{1}$ ) da ilustração

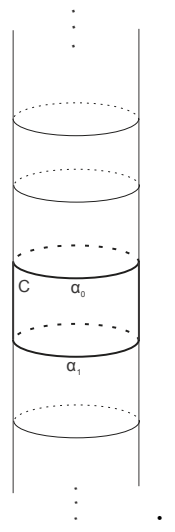

Sem perda de generalidade, podemos considerar a região $C$ para fazer modificações por homotopias, pois basta repetir analogamente estas modificações em cada cópia de $C$ em $\bar{T}$.

Lema 1.2.1. Seja uma aplicação $f: T^{2} \rightarrow W$ contínua e fortemente sobrejetiva com $l=1\left(f_{\pi}\right.$ sobrejetiva). Então existe $g \in[f]$ tal que $g^{-1}\left(W \backslash S^{1}\right)$ é uma subvariedade de $T^{2}$ 
homeomorfa $a \bigsqcup_{t=1}^{n} B_{t}^{2}$, onde $n \in \mathbb{N}$, e $B_{t}^{2}$ é o interior de discos $D_{t}^{2}$ onde $\partial D_{t}^{2} \simeq S_{t}^{1}$ e se $i \neq j S_{i}^{1} \cap S_{j}^{1}=\emptyset$. Além disso, $g\left(S_{t}^{1}\right)=P$ e $\left.g\right|_{\left(D_{t}^{2}, \partial D_{t}^{2}\right)}:\left(D_{t}^{2}, \partial D_{t}^{2}\right) \rightarrow\left(S^{2}, P\right)$ tem grau \pm 1 .

Demonstração: Assim como na demonstração do Lema 1.1.1 podemos usar os conceitos de diferenciabilidade local. Então tomemos um valor regular $y \in S^{2} \subset W$. Então pelo Teorema de Sard y possui uma vizinhança 2-Euclidiana $V \subset S^{2}$ tal que $V$ não possua valor crítico. Como $S$ é compacto e y é valor regular $f^{-1}(y)$ é um número finito de pontos $\left\{x_{1}, \ldots, x_{t}\right\} \subset T^{2}$. Considere uma vizinhança $B_{1} \subset T^{2}$ de $x_{1}$ homeomorfa ao disco aberto de dimensão 2 tal que $f\left(B_{1}\right) \subset V$ e $\partial B_{1} \cap f^{-1}(y)=\emptyset$. Pelo Lema 5.3 de [6] tem-se que $f$ pode ser deformada por homotopia em uma aplicação $f^{1}: T^{2} \rightarrow W$ tal que $\left.f^{1}\right|_{\left(T^{2} \backslash B_{1}\right)}=\left.f\right|_{\left(T^{2} \backslash B_{1}\right)},\left(f^{1}\right)^{-1}(y) \cap B_{1}=\left\{x_{1}\right\}$ e $f^{1}\left(B_{1}\right) \subset V$.

Repetindo o processo acima para $x_{2}$ e depois sucessivamente até $x_{t}$, existirá $B_{1}, \ldots, B_{t}$ vizinhanças disjuntas, homeomorfas ao disco aberto de dimensão 2 , de respectivamente $x_{1}, \ldots, x_{t}$ tais que $f^{t}: T^{2} \rightarrow W$ satisfaz:

1) $\left.f^{t}\right|_{\left(T^{2} \backslash\left(B_{1} \cup \ldots \cup B_{t}\right)\right)}=\left.f\right|_{\left(T^{2} \backslash\left(B_{1} \cup \ldots \cup B_{t}\right)\right)}$,

2) $\left(f^{t}\right)^{-1}(y) \cap B_{i}=\left\{x_{i}\right\}$ e $f^{t}\left(B_{i}\right) \subset V$, para todo $i \in\{1, \ldots, t\}$,

3) $f^{t} \cong f$.

Podemos tomar um caminho simples $c: I \rightarrow W$, tal que $c$ conecta todos $B_{i}$ e $c(I) \subset$ $V \backslash \bigsqcup_{i=1}^{t} \stackrel{\circ}{B_{i}}$. Sem perda de generalidade podemos supor $V=\bigsqcup_{i=1}^{t} B_{i} \cup(c(I))$.

Considere a homotopia $H: W \times I \rightarrow W$ tal que $H(x, 0)=x$ e $H(x, 1)=r(x)$ onde $r: W \rightarrow W$ é uma aplicação tal que $r(V)=W \backslash S^{1}$ e $\left.r\right|_{S^{1}}=\left.\left(I d_{W}\right)\right|_{S^{1}}$, isto é $r$ retrai todo aberto $V$ em $W \backslash S^{1}$ e o resto mantém a identidade, como na ilustração 1.1 .

Assim $f^{t} \cong f=H(., 0) \circ f \cong H(., 1) \circ f=r \circ f \cong r \circ f^{t}$.

Portanto a menos de homotopia $f^{-1}\left(W \backslash S^{1}\right)$ é uma subvariedade de $T^{2}$ homeomorfa a $\bigsqcup_{t=0}^{n} B_{t}^{2}$, onde $n$ é finito, $B_{t}^{2}$ são discos abertos de dimensão 2. Além disso, por construção, $B_{t}^{2}$ é o interior de discos $D_{t}^{2}$ tal que os bordos $\partial D_{t}^{2} \simeq S_{t}^{1}$ são dois a dois disjuntos, onde $f\left(S_{t}^{1}\right)=P$ e $f \mid D_{t}^{2}:\left(D_{t}^{2}, S_{t}^{1}\right) \rightarrow\left(S^{2}, P\right)$ tem grau \pm 1. 
Pelo diagrama comutativo 1.2 e pelo Lema 1.2.1, a menos de homotopia, $(f \circ \bar{p})^{-1}\left(W \backslash S^{1}\right) \cap C=\bigsqcup_{t=0}^{n} \bar{B}_{t}^{2}$, onde $\bar{B}_{t}^{2}=\bar{D}_{t}^{2}$ tal que $\bar{D}_{t}^{2}$ são discos fechados com $\partial \bar{D}_{t}^{2}=\bar{S}_{t}^{1}$ satisfazendo $(f \circ \bar{p})\left(\bar{S}_{t}^{1}\right)=P,\left.(f \circ \bar{p})\right|_{\left(\bar{D}_{t}^{2}, \bar{S}_{t}^{1}\right)}$ tem grau \pm 1 com $t \in\{1, \ldots, n\}$.

Da mesma forma como feito na seção1.1podemos juntar os discos $\bar{D}_{t_{i}}^{2}$ tal que $\widetilde{f}_{\bar{p}}\left(\bar{D}_{t_{i}}^{2}\right)=$ $S_{i}^{2}$ em um único disco e o grau neste novo disco é a soma dos graus, isto é, vale o Lema seguinte.

Lema 1.2.2. Sejam uma aplicação $f: T^{2} \rightarrow S^{1} \subset W$ contínua, fortemente sobrejetiva com $l=1$ e $\widetilde{f}_{\bar{p}}: \bar{T} \rightarrow \widetilde{W}$ como antes. Então existe $\widetilde{g}_{\bar{p}} \simeq \widetilde{f}_{\bar{p}}$ tal que $\widetilde{g}_{\bar{p}}^{-1}\left(S_{i}^{2} \backslash\{i\}\right) \cap C \cong \bar{B}_{i_{0}}^{2}$ ou $\widetilde{g}_{\bar{p}}^{-1}\left(S_{i}^{2} \backslash\{i\}\right)=\emptyset$, onde $B_{i_{0}}^{2}$ são interiores de discos fechados $\bar{D}_{i_{0}}^{2}$ com $\partial \bar{D}_{i_{0}}^{2} \simeq \bar{S}_{i_{0}}^{2}$ e para $i \neq j, \bar{S}_{i_{0}}^{1} \cap \bar{S}_{j_{0}}^{1}=\emptyset$. Além disso, $\widetilde{g}_{\bar{p}}\left(\bar{S}_{i_{0}}^{1}\right)=i$ e $i \in\{0, \ldots, n\}$.

Demonstração: Desde que $\widetilde{f}_{\bar{p}}(C)$ é compacto existe um primeiro índice $k$ e um último índice $k+n$ tal que $S_{k}^{2}$ e $S_{k+n}^{2}$ pertence a imagem de $\widetilde{f}_{\bar{p}}$.

Pelo Lema 1.2.1, $f^{-1}\left(W \backslash S^{1}\right)$ é uma subvariedade de $T^{2}$ homeomorfa a $\bigsqcup_{t=0}^{n} B_{t}^{2}$, onde $n$ é finito, $B_{t}^{2}$ são discos abertos de dimensão 2 , além disso, por construção, $B_{t}^{2}$ é o interior de discos $D_{t}^{2}$ tal que os bordos $\partial D_{t}^{2} \simeq S_{t}^{1}$ são dois a dois disjuntos, onde $f\left(S_{t}^{1}\right)=P$ e $f \mid D_{t}^{2}:\left(D_{t}^{2}, S_{t}^{1}\right) \rightarrow\left(S^{2}, P\right)$ tem grau \pm 1.

Portanto para cada $j \in\{k, \ldots, k+n\}, \tilde{f}_{\bar{p}}^{-1}\left(S_{j}^{2} \backslash\{j\}\right)$ é uma subcoleção $\bigsqcup_{l=n_{j}}^{n_{j}+r} B_{l}^{2} \subset \bigsqcup_{t=0}^{n} B_{t}^{2}$.

Fixemos $j \in\{k, \ldots, k+n\}$, juntaremos os discos $\bigsqcup_{l=n_{j}}^{n_{j}+r} D_{l}^{2}$ em um único disco.

Como $T^{2} \backslash f^{-1}\left(W \backslash S^{1}-\{P\}\right)$ é conexo por caminhos quaisquer dois pontos podem ser ligados por um caminho em $T^{2} \backslash f^{-1}\left(W \backslash S^{1}-\{p\}\right)$. Portanto tomemos um caminho simples $c: I \rightarrow T^{2}$ tal que vale as seguintes propriedades:

i) $c(I) \subset T^{2} \backslash f^{-1}\left(W \backslash S^{1}-\{P\}\right)$.

ii) $c(0) \in S_{n_{j}}^{1} \subset \bigsqcup_{l=n_{j}}^{n_{j}+r} S_{l}^{1}, c\left(\frac{1}{r-1}\right) \in S_{n_{j}+1}^{1} \subset \bigsqcup_{l=n_{j}}^{n_{j}+r} S_{l}^{1}, \ldots, c\left(\frac{r-2}{r-1}\right) S_{n_{j}+r-1}^{1} \subset \bigsqcup_{l=n_{j}}^{n_{j}+r} S_{l}^{1} \mathrm{e}$ $c(1) \in S_{n_{j}+r}^{1} \subset \bigsqcup_{l=n_{j}}^{n_{j}+r} S_{l}^{1}$.

Por construção do caminho $c, \widetilde{f}_{\bar{p}} \circ c \in \mathbb{R} \subset \widetilde{W}$. Como $I$ é compacto segue que $\tilde{f}_{\bar{p}} \circ c(I)$ também é compacto tal que $\tilde{f}_{\bar{p}} \circ c(0)=\tilde{f}_{\bar{p}} \circ c(1)=j$. Portanto a menos de 
homotopia, podemos considerar que $\widetilde{f}_{\bar{p}} \circ c(I)=j$. Logo a menos de homotopia existe um único disco $D_{j} \subset T^{2}$ tal que $\widetilde{f}_{\bar{p}}\left(D_{j}\right) \subset S_{j}^{2}, \widetilde{f}_{\bar{p}}\left(\partial D_{j}\right)=j$ e o grau de $\left.\widetilde{f}\right|_{D_{j}}$ é a soma dos graus deg $\widetilde{f}_{\bar{p}}\left|D_{1}^{2}+\ldots+\operatorname{deg} \widetilde{f}_{\bar{p}}\right| D_{n_{j}}^{2}$. Se esta soma for zero então, a menos de homotopia, $\widetilde{f}_{\bar{p}}\left(D_{j}\right)=j$.

Repetimos este processo de junção para cada índice $j \in\{k, \ldots, k+n\}$.

Portanto como $\widetilde{f}_{\bar{p}} \circ p=\bar{p} \circ f$, a menos de homotopia, $f$ é como queríamos.

Este Lema junta certos discos do Lema $1.2 .1 \mathrm{em}$ um único disco $D_{i}$ tal que $\left.\widetilde{f}_{\bar{p}}\right|_{\left(D_{i}, \partial D_{i}\right)}$ tenha grau igual a soma dos graus anteriores. Eventualmente esta soma pode ser nula. Mas quando isto acontecer podemos fazer uma homotopia tal que a imagem de $\widetilde{f}_{\bar{p}}$ não atinge $S_{i}^{2} \backslash\{i\}$.

Como já vimos a imagem de $\widetilde{f}_{\bar{p}}$ é determinada pela imagem da região $C$. Agora $\widetilde{f}_{\bar{p}}(C)$ é compacta, então existem inteiros $k^{+}$e $k^{-}$tal que $\left.\widetilde{f}_{\bar{p}}\right|_{\left(D_{k^{+}}, \partial D_{k^{+}}\right)}$e $\left.\widetilde{f}_{\bar{p}}\right|_{\left(D_{k^{-}}, \partial D_{k^{-}}\right)}$têm graus diferentes de zero.

A menos de escolha de ponto base de $\widetilde{W}$, como já vimos antes, podemos considerar $k^{-}=0$ e $k^{+}=n$, para algum $n \in \mathbb{N}$.

Logo a menos de homotopia $\left.\widetilde{f}_{\bar{p}}\right|_{C}$ é da seguinte forma

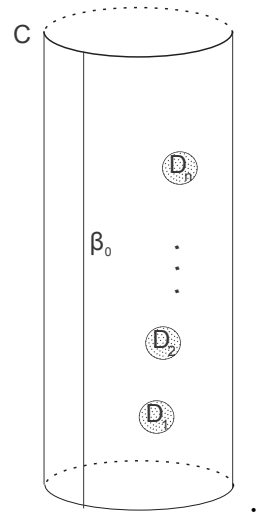

onde $D_{i}$ recobre $S_{i}^{2}$ com grau não nulo, denotaremos este grau por $a_{i}$ e a aresta $\beta_{0}$ recobre o intervalo $[0,1] \subset \widetilde{W}$ injetivamente.

Podemos considerar $\partial D_{0} \cap \alpha_{0} \neq \emptyset$, pois caso contrário basta tomar um caminho simples $d: I \rightarrow \stackrel{\circ}{C} \backslash\left(\bigsqcup_{t=0}^{j} \bar{B}_{i_{j}}^{2}\right)$ tal que $d(0) \in \partial \bar{D}_{0}$ e $d(1) \in \beta_{0}$ e fazer homotopias numa vizinhança da imagem de $d$ tal que $\widetilde{f}_{\bar{p}}(d(I))=0 \in \mathbb{Z} \subset \widetilde{W}$. 
Consideremos um caminho simples $c: I \rightarrow \stackrel{\circ}{C} \backslash\left(\bigsqcup_{t=0}^{j} \bar{B}_{i_{j}}^{2}\right)$ tal que

- $c(0) \in \partial D_{0}$ e $c\left(\frac{i}{n}\right) \in \partial D_{i}$

- $f(c((i-1, i)))=W \backslash\{P\} \mathrm{e}$

- $\left.f\right|_{c((i-1, i))}$ é injetiva,

onde $i \in\{1, \ldots, n\}$.

Seja $V \subset C$ uma vizinhança tubular de $c(I) \cup\left(\bigsqcup_{i=0}^{n} B_{i}\right)$ como segue

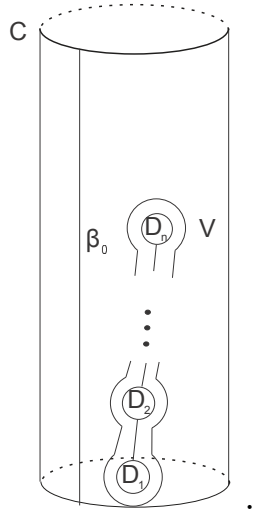

Fazemos uma mudança por homotopia dentro desta vizinhança $V$ (se necessário tome uma vizinhança $V^{\prime} \subset V$ tal que $\left.\partial V^{\prime} \cap \partial V=\emptyset\right)$ tal que $V \simeq D \subset C$ tal que $\widetilde{f}_{\bar{p}}(\partial D)=0 \in$ $\mathbb{Z} \subset \widetilde{W}$

Utilizando os resultados do seção 1.1, a aplicação $\left.p \circ \widetilde{f}_{\bar{p}}\right|_{(D, \partial D)}:(D, \partial D) \rightarrow(W, P)$ é homotópica a uma aplicação especial (como aplicação de $S^{2}$ em $W$ ) e manteremos as mesmas notação de $\widetilde{f}_{\bar{p}}$ para estas aplicações homotópicas.

A imagem $\bar{p}(D)$ é um disco $D^{\prime}$ de dimensão 2 no toro $T^{2}$.

Consideremos a aplicação $\left.f\right|_{\left(T^{2} \backslash D^{\prime}\right)}:\left(T^{2} \backslash D^{\prime}\right) \rightarrow S^{1}$ e definimos uma aplicação $g$ : $T^{2} \rightarrow S^{1}$ por

$$
g(x)=\left\{\begin{array}{ccc}
f(x) & \text { se } & x \in\left(T^{2} \backslash D^{\prime}\right) \\
P & \text { se } & x \in D^{\prime}
\end{array}\right.
$$

Assim como feito anteriormente, podemos usar a Teoria de Morse em $S^{1}$, para determinar uma aplicação $g^{\prime}: T^{2} \rightarrow S^{1}$ tal que $g\left(D^{\prime}\right)=P$ e $g^{\prime}$ seja aplicação de Morse, onde $g^{\prime-1}(x)=S^{1} \subset T^{2}$ para qulaquer $x \in S^{1} \backslash\{P\}$.

Definimos $g: T^{2} \rightarrow W$ por 
- $\left.g\right|_{\left(T^{2} \backslash D^{\prime}\right)}=\left.g^{\prime}\right|_{T^{2} \backslash D^{\prime}}$

- $\left.g\right|_{D}=\left.(f \circ \bar{p})\right|_{D}$

Obtemos uma aplicação $g \simeq f$, onde $g$ é especial com $p_{[g]}(t)=p_{[f]}(t)=p_{\left[\left.f\right|_{(D, \partial D)}\right](t)} \mathrm{e}$ $f_{\pi}\left(\pi_{1}\left(T^{2}\right)\right)=<f_{\pi}(\alpha)>=<1>=\mathbb{Z}$, isto é, $l=1$.

Agora só falta analisar o caso em que $f_{\pi} \neq 0$ e $f_{\pi}$ não é sobrejetora, isto é, $l \geq 2$.

Neste caso a imagem $f_{\pi}\left(\pi_{1}\left(T^{2}\right)\right)$ é um subgrupo de $\mathbb{Z}$, logo a imagem é isomorfa a $\mathbb{Z}$. Seja $l \in \mathbb{N}$ o gerador desta imagem.

A menos de escolha de geradores podemos supor que $\left\langle\alpha>\oplus\langle\beta\rangle=\mathbb{Z} \oplus \mathbb{Z} \cong \pi_{1}\left(T^{2}\right)\right.$ tal que $f_{\pi}(\alpha)=l$ e $f_{\pi}(\beta)=0$.

Da mesma maneira que antes, podemos supor que $\alpha \subset f^{-1}(x)$, onde $x$ é valor regular e que $\alpha$ e $\beta$ são da forma como mostrado na figura abaixo

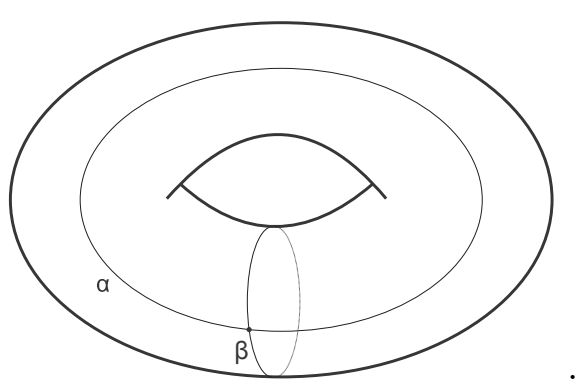

Sejam $\bar{T}$ o espaço de recobrimento cíclico infinito de $T^{2}$ relativo ao $\operatorname{ker}\left(f_{\pi}\right)$ e $\widetilde{f}_{\bar{p}}: \bar{T} \rightarrow$ $\widetilde{W}$ o levantamento de $f \circ \bar{p}$ tal que o diagrama

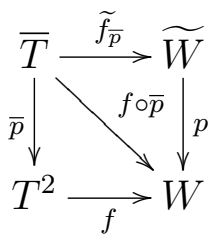

é comutativo, onde $\bar{p}: \bar{T} \rightarrow T^{2}$ e $p: \widetilde{W} \rightarrow W$ são aplicações de recobrimento.

Como $\bar{T}$ é o recobrimento cíclico infinito de $T^{2}$ existe uma região básica, que denotaremos por $C$, tal que é suficiente fazer alterações (por homotopias) na aplicação $\widetilde{f}_{\bar{p}}$ restrita a $C$ e depois repetir o processo para todas as outras cópias de $C \subset \bar{T}$. 
A região $C$ é um cilindro fechado, isto é, $S^{1} \times[-\epsilon, \epsilon]$ tal que $S^{1} \times\{-\epsilon\} \cup S^{1} \times\{\epsilon\} \subset$ $\bar{p}^{-1}(\beta)$ e $e \times[-\epsilon, \epsilon]=\bar{p}^{-1}(\alpha)$ para um ponto fixado $e \in S^{1}$, como na figura abaixo,

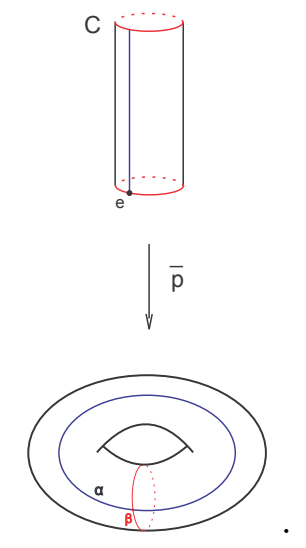

Assim, como antes, vamos nos concentrar somente nesta região $C$ para fazer modificações.

Observemos que fixado uma região $C$ e fixado um ponto $e \in S^{1}$ a imagem pela $\widetilde{f}_{\bar{p}}$ do conjunto $e \times[-\epsilon, \epsilon]$ é um intervalo $[j, j+l] \subset \mathbb{R} \subset \widetilde{W}$ e a imagem pela $\widetilde{f}_{\bar{p}}$ do conjunto $S^{1} \times\{\epsilon\}$ e $S^{1} \times\{-\epsilon\}$ são os pontos $j+l$ e $j$, respectivamente.

Podemos supor que a região $C$ seja tal que $j=0$, pois isto significa que escolhemos $j=0$ como sendo ponto base de $\widetilde{W}$ e já vimos que no caso de classes de homotopias livres de aplicações independe da escolha do ponto base. Denotaremos os seguintes conjuntos $S^{1} \times\{-\epsilon\}=\beta_{0}, S^{1} \times\{\epsilon\}=\beta_{1}$ e $e \times[-\epsilon, \epsilon]=\alpha_{0}$.

Usando as mesmas técnicas que no caso de $f_{\pi}$ ser sobrejetora, os conjuntos $C \cap \tilde{f}_{\bar{p}}^{-1}\left(S_{i}^{2} \backslash\{i\}\right)=B_{i}^{2}$ tal que $B_{i}^{2}$ são discos abertos de dimensão 2 que são interiores de discos disjuntos fechado $D_{i}^{2} \operatorname{com} \widetilde{f}_{\bar{p}}\left(\partial D_{i}^{2}=S_{i}^{1}\right)=i$ e $\left.\widetilde{f}_{\bar{p}}\right|_{\left(D_{i}^{2}, S_{i}^{1}\right)}$ tem grau diferente de zero.

Da mesma forma juntamos estes discos num único disco $D \subset \bar{T}$ tal que $\widetilde{f}_{\bar{p}}(\partial D)=0$ e $\left.p \circ \widetilde{f}_{\bar{p}}\right|_{(D, \partial D)}:(D, \partial D) \rightarrow(W, P)$ é uma aplicação especial (como aplicação de $S^{2}$ em $W$ ).

Sejam $D^{\prime}=\bar{p}(D)$ e a aplicação $\left.f\right|_{\left(T^{2} \backslash D^{\prime}\right)}:\left(T^{2} \backslash D^{\prime}\right) \rightarrow S^{1}$. Definimos uma aplicação $g: T^{2} \rightarrow S^{1}$ por

$$
g(x)=\left\{\begin{array}{ccc}
f(x) & \text { se } & x \in\left(T^{2} \backslash D^{\prime}\right) \\
P & \text { se } & x \in D^{\prime}
\end{array}\right.
$$

Assim como feito anteriormente, podemos usar a Teoria de Morse Circular, para de- 
terminar uma aplicação $g^{\prime}: T^{2} \rightarrow S^{1}$ tal que $g\left(D^{\prime}\right)=P, g^{\prime}$ seja aplicação de Morse e $g^{\prime} \simeq g$.

Observemos que como $\widetilde{f}_{\bar{p}}(C \backslash D)=[0, l]$ então $g_{\pi}^{\prime}\left(\pi_{1}\left(T^{2}\right)\right) \simeq \mathbb{Z}<l>\subset \mathbb{Z} \simeq \pi_{1}(W)$. Portanto $g^{\prime-1}(x)=\bigsqcup_{j=1}^{l} S_{j}^{1} \subset T^{2}$ para qualquer $x \in S^{1} \backslash\{P\}$.

Agora definimos a aplicação $g: T^{2} \rightarrow W$ por

- $\left.g\right|_{\left(T^{2} \backslash D^{\prime}\right)}=\left.g^{\prime}\right|_{T^{2} \backslash D^{\prime}}$

$-\left.g\right|_{D}=\left.(\bar{p} \circ f)\right|_{D}$

Concluímos com uma aplicação $g \simeq f$, onde $g$ é especial com $p_{[g]}(t)=p_{[f]}(t)=$ $p_{\left[\left.f\right|_{(D, \partial D)}\right](t)}$ e $f_{\pi}\left(\pi_{1}\left(T^{2}\right)\right)=<f_{\pi}(\alpha)>\simeq \mathbb{Z}<l>\subset \mathbb{Z} \simeq \pi_{1}(W)$.

Portanto a menos de homotopia temos $f$ uma aplicação especial e segue o teorema.

Teorema 1.2.1. Seja uma aplicação $f: T^{2} \rightarrow W$, então existe uma aplicação especial $g \in[f]$.

\subsection{Representação das classes de homotopia de aplica- ções de $T_{g}$ em $W$}

Sejam $T_{g}$ superfície fechada e orientável de gênus $g>1$ e uma aplicação $f: T_{g} \rightarrow W$.

Assim como feito nas seções anteriores, associaremos um polinômio a $[f]$ e a partir deste polinômio encontraremos um elemento $g \in[f]$ com boas propriedades.

\section{Polinômio associado a uma aplicação de $T_{g}$ em $W$}

Seja $f_{\pi}: \pi_{1}\left(T_{g}\right) \rightarrow \pi_{1}(W)$ o homomorfismo induzido por $f$ em grupos fundamentais.

Uma apresentação grupo fundamental de uma superfície $T_{g}$ fechada, conexa e orientável de gênus $g>1$ é dado por

$$
\pi_{1}\left(T_{g}\right)=<\alpha_{1}, \beta_{1}, \alpha_{2}, \beta_{2}, \ldots, \alpha_{g}, \beta_{g} ; \alpha_{1} \beta_{1} \alpha_{1}^{-1} \beta_{1}^{-1} \alpha_{2} \beta_{2} \alpha_{2}^{-1} \beta_{2}^{-1} \ldots \alpha_{g} \beta_{g} \alpha_{g}^{-1} \beta_{g}^{-1}>
$$

Uma superfície pode ser ilustrada como um polígono identificando os lados que são os 
geradores de $\pi_{1}\left(T_{g}\right)$ são ilustrado como abaixo

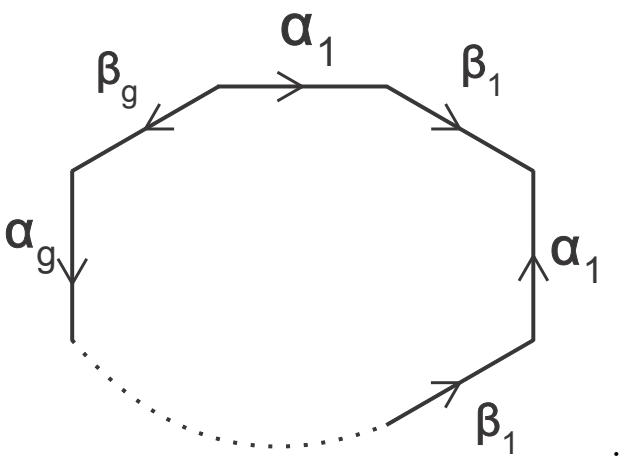

Quando $f_{\pi}=0$, então $f_{\pi}\left(\alpha_{i}\right)=f_{\pi}\left(\beta_{i}\right)=0$ para todo $i \in\{1, \ldots, g\}$. Caso $f_{\pi} \neq 0$, existe $l \in \mathbb{N}^{*}$ tal que $f_{\pi}\left(\pi_{1}\left(T_{g}\right)\right) \simeq \mathbb{Z}<l>\subset \mathbb{Z} \simeq \pi_{1}(W)$.

Vamos fazer assim como feito no caso do toro, isto é, fazer mudança de base nos geradores (no caso do toro geradores de $\pi_{1}\left(T^{2}\right)$ ), tal que somente um, a menos de homotopia, gerador é levado em $S^{1} \subset W$ e os outros, a menos de homotopia, é levado em $P \in W$.

Para isso, como $\pi_{1}\left(T_{g}\right)$ não tem uma base, compomos o homomorfismo induzido por $f$ em grupos fundamentais com a aplicação $\tau: \pi_{1}\left(T_{g}\right) \rightarrow H_{1}\left(T_{g}\right)$

Assim como foi feito no caso do toro, podemos considerar os geradores de $\pi_{1}\left(T_{g}\right)$ satisfazendo $f_{\pi}\left(\alpha_{1}\right)=l$ e $f_{\pi}\left(\alpha_{i}\right)=f_{\pi}\left(\beta_{j}\right)=0$, onde $i \in\{2, \ldots, g\}$ e $j \in\{1, \ldots, g\}$.

Identificamos o conjunto $T_{g} \backslash\left(\bigsqcup_{i=1}^{g} \beta_{i} \bigsqcup_{i=2}^{g} \alpha_{i}\right) \operatorname{com} S^{2} \backslash \bigsqcup_{i=1}^{2 g-1} D_{i}$, onde $D_{i}$ são discos de dimensão 2 e $\alpha_{i}, \beta_{i}$ são os geradores de $\pi_{1}\left(T_{g}\right)$.

Definimos $f_{S^{2}}: S^{2} \rightarrow W$ da seguinte maneira;

$$
f_{S^{2}}(x)= \begin{cases}P & , \text { se } x \in \bigsqcup_{i=1}^{2 g-1} D_{i} \\ f(x) & , \text { se } x \in S^{2} \backslash\left(\bigsqcup_{i=1}^{2 g-1} D_{i}\right)\end{cases}
$$

Sejam $f: T_{g} \rightarrow W$ e $f_{S^{2}}: S^{2} \rightarrow W$ como acima.

Definição 1.3.1. O polinômio $p_{\left[f_{S^{2}}\right](t)}$ será o polinômio associado à $[f]$ e denotaremos por $p_{[f]}(t)$.

Construção de uma aplicação a partir de $p(t)$ e $l \in \mathbb{N}$ 
Dada uma superfície fechada e orientável $T_{g}$ de gênus $g \geq 1$, então pelo teorema de classificação de superfície $T_{g} \cong \underbrace{T^{2} \# \ldots \# T^{2}}_{g-\text { vezes }}$, onde \# é a soma conexa.

Portanto o recobrimento cíclico infinito $\overline{T_{g}}$ de uma superfície $T_{g}$ é como segue

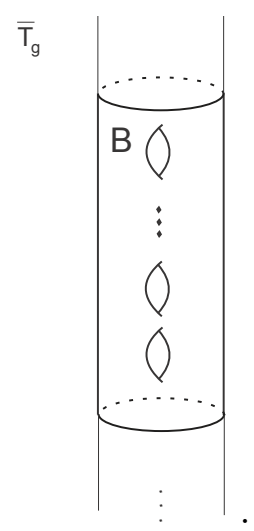

onde a região destacada é uma região básica e será denotado por $B$.

Para definir uma aplicação $\bar{f}_{\bar{p}}: \overline{T_{g}} \rightarrow \widetilde{W}$ basta defini-lá somente na região $B$ e depois estender $\bar{f}_{\bar{p}}$ nas outras cópias de $B$, por isso $B$ é chamada de região básica.

Sejam um polinômio $q(t)=a_{0}+\ldots+a^{n} t^{n}$ e um número $l \in \mathbb{N}$.

Considere a aplicação $\bar{f}_{\bar{p}}$ definida na região $B$ da seguinte maneira

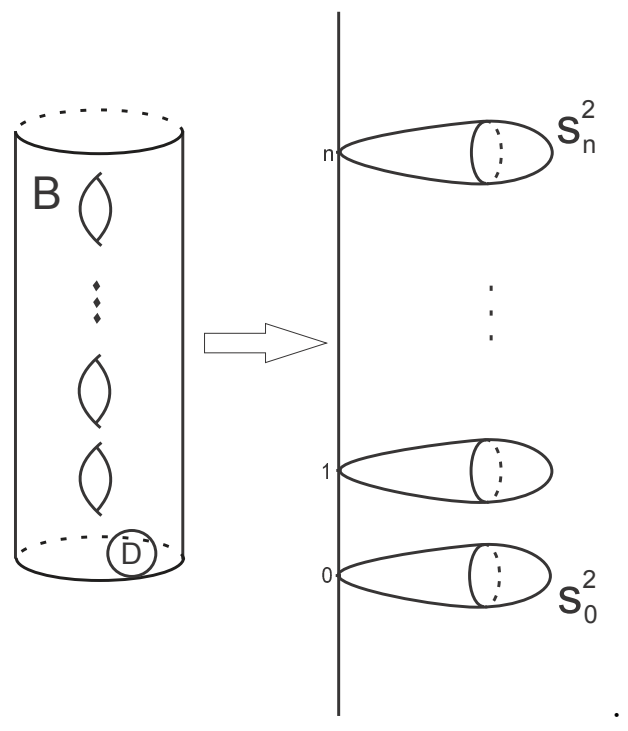

onde $\bar{f}_{\bar{p}}(\partial D)=0 \in \mathbb{Z} \subset(\widetilde{W}, 0),\left.\bar{f}_{\bar{p}}\right|_{(D, \partial D)}:(D, \partial D) \rightarrow \widetilde{W}$ é construída a partir do polinômio $q(t)$ como na seção 1.1 e a região $\bar{f}_{B \backslash D}$ é uma sobrejeção no intervalo $[0, l]$.

Por fim, definimos a aplicação $f: T_{g} \rightarrow W$ por 


$$
f(x)=p \circ \bar{f}_{\bar{p}}(\bar{x})
$$

onde $\bar{p}: \overline{T_{g}} \rightarrow T_{g}$ é recobrimento, $p: \widetilde{W} \rightarrow W$ é recobrimento universal e $\bar{x}=\bar{p}^{-1}(x) \cap B$.

Portanto por construção $p_{[f]}(t)=q(t)$ e $f_{\pi}\left(\pi_{1}\left(T_{g}\right)\right)=<l>\subset \mathbb{Z}$, onde $f_{\pi}\left(\alpha_{1}\right)=l$ e $f_{\pi}\left(\alpha_{i}\right)=f_{\pi}\left(\beta_{j}\right)=0$ para $i \in\{2, \ldots, g\}$ e $j \in\{1, \ldots, g\}$.

Definição 1.3.2. Uma aplicação $f: T_{g} \rightarrow W$ será chamada especial se ela pode ser construída como acima, isto é, a partir do polinômio $p_{[f]}(t)$ e de $l$, onde $l \in \mathbb{N}$ é um gerador de $f_{\pi}\left(\pi_{1}\left(T_{g}\right)\right)$.

\section{Encontrando uma aplicação especial $g \in[f]$}

Para encontrar uma aplicação especial $g \in[f]$ seguiremos os passos feitos na seção 1.2 .

Seja $T_{g}$ superfície fechada, conexa e orientável de gênus $g>1$.

No caso em que a classe de homotopia $[f] \in\left[T_{g}, W\right]$ é tal que $f_{\pi}: \pi_{1}\left(T_{g}\right) \rightarrow \pi_{1}(W)$ é a aplicação nula pode ocorrer as seguintes situações:

- Se, a menos de homotopia, $f\left(T_{g}\right) \subset W \backslash S^{1}$ ou $f\left(T_{g}\right) \subset W \backslash S^{2}$, então $f$ é homotópica a uma função constante.

- Se, a menos de homotopia, $f\left(T_{g}\right)=S^{2} \subset W$, então usando as técnicas dos lemas 1.2.2 e 1.2.1, a menos de homotopia, temos $f^{-1}(P)=\underbrace{S^{1} \vee \ldots \vee S^{1}}_{2 g-\text { vezes }}$ e $f^{-1}\left(S^{2} \backslash\{P\}\right)=B^{2}$ tal que $T_{g} \backslash B^{2}=\underbrace{S^{1} \vee \ldots \vee S^{1}}_{2 g \text {-vezes }}$ e $\left.f\right|_{\left(B^{2}, S \backslash B^{2}\right)}$ recobre $S^{2}$ com grau diferente de zero.

- Se $f_{\pi}=0$ e, a menos de homotopia, $f\left(T_{g}\right)=S^{1} \subset W$, então $f$ é homotópica a uma aplicação constante.

- Se $f_{\pi}=0$ e para toda $g \in[f], g$ é sobrejetora. Então $f$ pode ser fatorada por $S^{2}$ tal que o diagrama

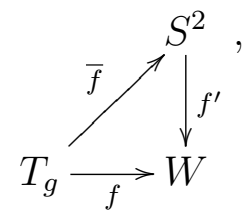

comuta. Logo $f$ é homotópica a uma aplicação $g: T_{g} \rightarrow W$ tal que $\bar{g}(\underbrace{S^{1} \vee \ldots \vee S^{1}}_{2 g-v e z e s})=$ 
$P \in S^{2},\left.\bar{g}\right|_{B^{2}}$ é uma bijeção, onde $B^{2}=T_{g} \backslash \underbrace{S^{1} \vee \ldots \vee S^{1}}_{2 g-\text { vezes }}$ e $g^{\prime}$ é representada como na seção anterior pelo seu polinômio $p_{\left[g^{\prime}\right]}(t)=p_{[f]}(t)$.

Quando uma aplicação $f: T_{g} \rightarrow W$ é tal que $f_{\pi} \neq 0$, temos o subgrupo $\operatorname{Im}\left(f_{\pi}\right) \subset \mathbb{Z}$, logo existe $l \in \mathbb{N}$ tal que $\operatorname{Im}\left(f_{\pi}\right) \simeq \mathbb{Z}<l>\subset \mathbb{Z} \simeq \pi_{1}(W)$.

No caso em que $f\left(T_{g}\right)=S^{1} \subset W$ usamos resultados da Teoria de Morse Circular par encontrar uma aplicação especial.

Portanto, daqui em diante, nesta seção, consideraremos $f$ fortemente sobrejetiva.

Primeiramente vamos supor que $l=1$ e da mesma forma como foi feito na seção anterior, podemos supor, sem perda de generalidade, que $f_{\pi}\left(\alpha_{1}\right)=1$ e $f_{\pi}(\gamma)=0$, onde $\gamma \in \pi_{1}\left(T_{g}\right) \backslash\left\{\alpha_{1}\right\}$.

Seja $\overline{T_{g}}$ o espaço de recobrimento de $T_{g}$ relativo ao $\operatorname{ker}\left(f_{\pi}\right)$, isto é, um recobrimento cíclico infinito de $T_{g}$ e temos o diagrama

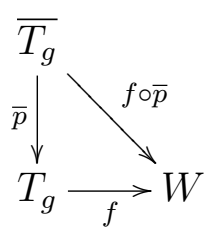

comutativo. O espaço topológico $\overline{T_{g}}$ é como na figura 1.3

Por construção do recobrimento $\overline{T_{g}}$, a imagem $(f \circ \bar{p})_{\pi}\left(\pi_{1}\left(\overline{T_{g}}\right)\right)$ é nula. Portanto a aplicação $f \circ \bar{p}$ se levanta ao recobrimento universal $\widetilde{W}$, isto é, o diagrama

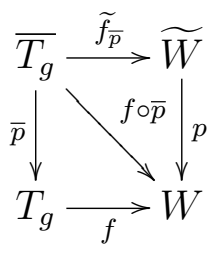

é comutativo.

Faremos homotopias na aplicação $\widetilde{f}_{\bar{p}}$ para encontrar $\widetilde{g}_{\bar{p}} \simeq \widetilde{f}_{\bar{p}}$ tal que $\widetilde{g}_{\bar{p}}$ irá induzir uma aplicação $g \simeq f$, onde $g$ é especial.

Consideremos $B$ a região destacada na ilustração 1.3 que é uma região básica para fazer as modificações por homotopias, pois basta repetir analogamente estas modificações em cada cópia de $B$ em $\overline{T_{g}}$. 
Lema 1.3.1. Seja $f: T_{g} \rightarrow W$ aplicação contínua, fortemente sobrejetiva com $l=1$. Então existe $g \in[f]$ tal que $g^{-1}\left(W \backslash S^{1}\right)$ é uma subvariedade de $T_{g}$ homeomorfa $a \bigsqcup_{t=1}^{n} B_{t}^{2}$, onde $n$ é finito, $B_{t}^{2}$ são discos abertos de dimensãa 2 e $B_{t}^{2}$ é o interior de discos $D_{t}^{2}$ onde $\partial D_{t}^{2} \simeq S_{t}^{1}$ e se $i \neq j, S_{i}^{1} \cap S_{j}^{1}=\emptyset$. Além disso, $g\left(S_{t}^{1}\right)=P$ e $g \mid D_{t}^{2}:\left(D_{t}^{2}, \partial D_{t}^{2}\right) \rightarrow\left(S^{2}, P\right)$ tem grau \pm 1 .

Demonstração: A demonstração é análoga a demonstração do Lema 1.2.1,

Pelo diagrama comutativo 1.3 e pelo lema 1.3.1 a menos de homotopia, $(f \circ \bar{p})^{-1}\left(W \backslash S^{1}\right) \cap B=\bigsqcup_{t=0}^{n} \bar{B}_{t}^{2}$, onde $n$ é finito, $\bar{B}_{t}^{2}$ são discos abertos de dimensão 2 e $\bar{B}_{t}^{2}$ é o interior de discos disjuntos dois a dois $\bar{D}_{t}^{2}$ onde $\partial \bar{D}_{t}^{2} \simeq \bar{S}_{t}^{1}$. Além disso, $(f \circ \bar{p})\left(\bar{S}_{t}^{1}\right)=P$ e $\left.(f \circ \bar{p})\right|_{\left(\bar{B}_{t}^{2}, \bar{S}_{t}^{1}\right)}$ tem grau \pm 1.

Lema 1.3.2. Sejam $f: T_{g} \rightarrow S^{1} \subset W$ aplicação contínua, fortemente sobrejetiva com $l=1$ e ${\widetilde{f_{\bar{p}}}}_{\bar{p}}: \overline{T_{g}} \rightarrow \widetilde{W}$ como antes. Então existe $\widetilde{g}_{\bar{p}} \simeq \widetilde{f}_{\bar{p}}$ tal que $\widetilde{g}_{\bar{p}}^{-1}\left(S_{i}^{2} \backslash\{i\}\right) \cap B \cong \bar{B}_{i_{0}}^{2}$ ou $\widetilde{g}_{\bar{p}}^{-1}\left(S_{i}^{2} \backslash\{i\}\right)=\emptyset$, onde $\bar{B}_{i_{0}}^{2}$ são discos abertos de dimensão 2 que são interiores de discos fechados $\bar{D}_{i_{0}}^{2}$ com $\partial \bar{D}_{i_{0}}^{2} \simeq \bar{S}_{i_{0}}^{2}$ e, para $i \neq j, S_{i_{0}}^{1} \cap S_{j_{0}}^{1}=\emptyset$. Além disso, $\widetilde{g}_{\bar{p}}\left(\bar{S}_{i_{0}}\right)=P e$ $i \in\{0, \ldots, n\}$.

Demonstração: Análoga a demonstração do Lema 1.2.2.

Este lema junta certos discos do Lema $1.3 .1 \mathrm{em}$ um único disco $D_{i}$ tal que $\left.\widetilde{f}_{\bar{p}}\right|_{\left(D_{i}, \partial D_{i}\right)}$ tenha grau igual a soma dos graus anteriores. Eventualmente esta soma pode ser nula. Mas quando isto acontecer podemos fazer uma homotopia tal que a imagem de $\widetilde{f}_{\bar{p}}$ não atinge $S_{i}^{2} \backslash\{i\}$.

Como já vimos a imagem de $\widetilde{f}_{\bar{p}}$ é determinada pela imagem da região $B$. Agora $\widetilde{f}_{\bar{p}}(B)$ é compacta, então existem inteiros $k^{+}$e $k^{-}$tal que $\left.\widetilde{f}_{\bar{p}}\right|_{\left(D_{k^{+}}, \partial D_{k^{+}}\right)}$e $\left.\widetilde{f}_{\bar{p}}\right|_{\left(D_{k^{-}}, \partial D_{k^{-}}\right)}$têm graus diferentes de zero.

Lembramos que o conjunto $\left[T_{g}, W\right]$ independe da escolha do ponto base de $\widetilde{W}$. Então a menos de escolha de ponto base de $\widetilde{W}$, podemos consideras $k^{-}=0$ e $k^{+}=n$, para $\operatorname{algum} n \in \mathbb{N}$. 
Logo a menos de homotopia $\widetilde{f}_{\bar{p}}$ restrita ao conjunto $B$ é da seguinte forma

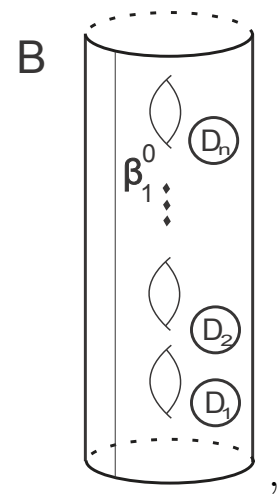

onde cada disco $D_{i}$ recobre $S_{i}^{2}$ com grau não nulo. Denotaremos este grau por $a_{i}$ e a aresta $\beta_{1}^{0}$ recobre o intervalo $[0,1] \subset \widetilde{W}$ injetivamente.

Podemos considerar $\partial D_{0} \cap \alpha_{0} \neq \emptyset$, pois caso contrário basta tomar um caminho simples $d: I \rightarrow \stackrel{\circ}{C} \backslash\left(\bigsqcup_{t=0}^{j} \bar{B}_{i_{j}}^{2}\right)$ tal que $d(0) \in \partial \bar{B}_{0}$ e $d(1) \in \beta_{1}^{0}$ e fazer homotopias numa vizinhança da imagem de $d$ tal que $f(d(I))=0$.

Tomemos um caminho simples $c: I \rightarrow \stackrel{\circ}{C} \backslash\left(\bigsqcup_{t=0}^{j} \bar{B}_{i_{j}}^{2}\right)$ tal que - $c(0) \in \partial D_{0}$ e $c\left(\frac{i}{n}\right) \in \partial D_{i}$

- $f(c((i-1, i)))=W \backslash\{P\} \mathrm{e}$

- $\left.f\right|_{c((i-1, i))}$ é injetiva,

onde $i \in\{1, \ldots, n\}$.

Seja $V \subset B$ uma vizinhança tubular de $c(I) \cup\left(\bigcup_{i=0}^{n} B_{i}\right)$ como segue

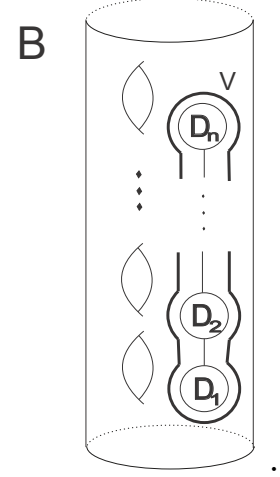

Podemos fazer uma homotopia, como foi feito no caso do toro, em $V$, tal que existe $D \subset B$ onde $\widetilde{f}_{\bar{p}}(\partial D)=0$. Utilizando os resultados do seção 1.1, a aplicação $\left.\widetilde{f}_{\bar{p}}\right|_{(D, \partial D)}$ : 
$(D, \partial D) \rightarrow(W, P)$ é homotópica a uma aplicação especial (como aplicação de $S^{2}$ em $W$ ) e manteremos as mesmas notação de $\widetilde{f}_{\bar{p}}$ para estas aplicações homotópicas.

A imagem $\bar{p}(D)$ é um disco $D^{\prime}$ de dimensão 2 na superfície $T_{g}$, assim a aplicação $\left.f\right|_{\left(T_{g} \backslash D^{\prime}\right)}:\left(T_{g} \backslash D^{\prime}\right) \rightarrow S^{1}$.

Consideremos a aplicação $f^{\prime}: T_{g} \rightarrow S^{1}$ dada por

$$
f^{\prime}(x)=\left\{\begin{array}{lll}
P & \text { se } & x \in D^{\prime} \\
f(x) & \text { se } & x \in T_{g} \backslash D^{\prime}
\end{array}\right.
$$

Da mesma forma como feito na seção 1.2. podemos usar resultados da Teoria de Morse Circular, para determinar uma aplicação de Morse $g^{\prime}: T_{g} \rightarrow S^{1}$ tal que $g^{\prime}\left(D^{\prime}\right)=\{P\}$ e $g^{\prime} \simeq f^{\prime}$

Sem perda de generalidade, $\left.\bar{p}\right|_{D^{\prime}}: D^{\prime} \rightarrow D$ é um difeomorfismo.

Definimos $g: T_{g} \rightarrow W$ por

- $\left.g\right|_{\left(T_{g} \backslash D^{\prime}\right)}=\left.g^{\prime}\right|_{T_{g} \backslash D^{\prime}}$

$-\left.g\right|_{D}=\left.\left(p \circ \bar{f}_{\bar{p}} \circ \bar{p}^{-1}\right)\right|_{D}$

Portanto $g \simeq f, g$ é especial com $p_{[g]}(t)=p_{[f]}(t)=p_{\left[\left.f\right|_{(D, \partial D)}\right](t)}$ e $f_{\pi}\left(\pi_{1}\left(T_{g}\right)\right)=<$ $f_{\pi}(\alpha)>=<1>=\mathbb{Z} \simeq \pi_{1}(W)$.

Agora só falta o caso em que $f_{\pi} \neq 0$ e $f_{\pi}$ não é sobrejetora, isto é, $l \geq 2$. Neste caso a imagem $f_{\pi}\left(\pi_{1}\left(T_{g}\right)\right)$ é um subgrupo de $\mathbb{Z}, \operatorname{logo}$ a imagem é isomorfa a $\mathbb{Z}$. Suponha $l \in \mathbb{N}$ gerador desta imagem.

A menos de escolha de geradores, temos $f_{\pi}\left(\pi_{1}\left(T_{g}\right)\right)=<l>\subset \mathbb{Z}$, onde $f_{\pi}\left(\alpha_{1}\right)=l$ e $f_{\pi}\left(\alpha_{i}\right)=f_{\pi}\left(\beta_{j}\right)=0$ para $i \in\{2, \ldots, g\}$ e $j \in\{1, \ldots, g\}$.

Sejam $\overline{T_{g}}$ o espaço de recobrimento cíclico infinito de $T_{g}$ relativo ao $k e r\left(f_{\pi}\right)$ e $\widetilde{f}_{\bar{p}}: \overline{T_{g}} \rightarrow$ $\widetilde{W}$ o levantamento de $f \circ \bar{p}$ tal que o diagrama 


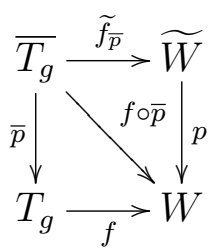

é comutativo, onde $\bar{p}: \overline{T_{g}} \rightarrow T_{g}$ e $p: \widetilde{W} \rightarrow W$ são aplicações de recobrimento.

Seja $B$ a região básica de $\overline{T_{g}}$ como antes, tal que é suficiente fazer alterações na aplicação $\widetilde{f}_{\bar{p}}$ restrita a $B$.

Assim como antes, vamos nos concentrar somente nesta região $B$ para fazer modificações por homotopias, pois basta repetir as modificações nas outras cópias de $B$.

Observemos que fixado uma região $B$ a imagem pela $\widetilde{f}_{\bar{p}}$ do conjunto $e \times[-\epsilon, \epsilon]$ é um intervalo $[j, j+l] \subset \mathbb{R} \subset \widetilde{W}$ e a imagem pela $\widetilde{f}_{\bar{p}}$ do conjunto $S^{1} \times\{\epsilon\}$ e $S^{1} \times\{-\epsilon\}$ são os pontos $j+l$ e $j$, respectivamente.

Como já vimos que o conjunto $\left[T_{g}, W\right]$ independe da escolha do ponto base no espaço $\widetilde{W}$, podemos supor que $j=0$ e denotaremos os seguintes conjuntos $S^{1} \times\{-\epsilon\}=\beta_{1}^{0}$, $S^{1} \times\{\epsilon\}=\beta_{1}$ e $e \times[-\epsilon, \epsilon]=\alpha_{0}$.

Assim como no caso de $f_{\pi}$ ser sobrejetora podemos modificar por homotopias a aplicação inicial tal que os conjuntos $B \cap \widetilde{f}_{\bar{p}}^{-1}\left(S_{i}^{2}\right)=\emptyset$ ou $B \cap \widetilde{f}_{\bar{p}}^{-1}\left(S_{i}^{2}\right)=\bar{B}_{i_{0}}^{2}$ tal que $\bar{B}_{i_{0}}^{2}$ são discos abertos de dimensão 2 que são interiores de discos disjuntos fechados $\bar{D}_{i_{0}}^{2} \operatorname{com} \partial \bar{D}_{i_{0}}^{2} \simeq \bar{S}_{i_{0}}^{1}$ e $\tilde{f}_{\bar{p}}\left(\bar{S}_{i_{0}}^{1}\right)=i$ e $i \in\{0, \ldots, n\}$ e $\left.\widetilde{f}_{\bar{p}}\right|_{\left(\bar{D}_{i}^{2}, \bar{S}_{i}^{1}\right)}$ tem grau diferente de zero e como no caso de $f_{\pi}$ ser sobrejetora unir estes discos $\bar{D}_{i_{0}}^{2}$ num único disco $D \subset B$ tal que $\widetilde{f}_{\bar{p}}(\partial D)=0$.

Observe que $\tilde{f}_{\bar{p}}(B \backslash D)=[0, l]$.

Utilizando os resultados do seção 1.1, a aplicação $\left.\widetilde{f}_{\bar{p}}\right|_{(D, \partial D)}:(D, \partial D) \rightarrow(W, P)$ é homotópica a uma aplicação especial (como aplicação de $S^{2}$ em $W$ ) e manteremos as mesmas notação de $\widetilde{f}_{\bar{p}}$ para estas aplicações homotópicas.

A imagem $\bar{p}(D)$ é um disco $D^{\prime}$ de dimensão 2 na superfície $T_{g}$ e sem perda de generalidade, $\left.\bar{p}\right|_{D^{\prime}}: D^{\prime} \rightarrow D$ é um difeomorfismo. Assim a aplicação $\left.f\right|_{\left(T_{g} \backslash D^{\prime}\right)}:\left(T_{g} \backslash D^{\prime}\right) \rightarrow S^{1}$.

Definimos a aplicação $f^{\prime}: T_{g} \rightarrow S^{1}$ dada por

$$
f^{\prime}(x)=\left\{\begin{array}{lll}
P & \text { se } & x \in D^{\prime} \\
f(x) & \text { se } & x \in T_{g} \backslash D^{\prime}
\end{array}\right.
$$


Da mesma forma como feito na seção [1.2, podemos usar resultados da Teoria de Morse Circular, para determinar uma aplicação de Morse $g^{\prime}: T_{g} \rightarrow S^{1}$ tal que $g^{\prime}\left(D^{\prime}\right)=P$ e $g^{\prime} \simeq f^{\prime}$

Definimos $g: T_{g} \rightarrow W$ por

- $\left.g\right|_{\left(T_{g} \backslash D^{\prime}\right)}=\left.g^{\prime}\right|_{T_{g} \backslash D^{\prime}}$

- $\left.g\right|_{D}=\left.\left(p \circ \bar{f}_{\bar{p}} \circ \bar{p}^{-1}\right)\right|_{D}$.

Portanto $g \simeq f, g$ é especial com $p_{[g]}(t)=p_{[f]}(t)=p_{\left[\left.f\right|_{(D, \partial D)}\right](t)}$ e $f_{\pi}\left(\pi_{1}\left(T_{g}\right)\right)=<$ $f_{\pi}(\alpha)>=\mathbb{Z}<l>\subset \mathbb{Z} \simeq \pi_{1}(W)$ e temos o seguinte teorema:

Teorema 1.3.1. Seja uma aplicação $f: T_{g} \rightarrow W$. Então existe $g \in[f]$ tal que $g$ é especial. 


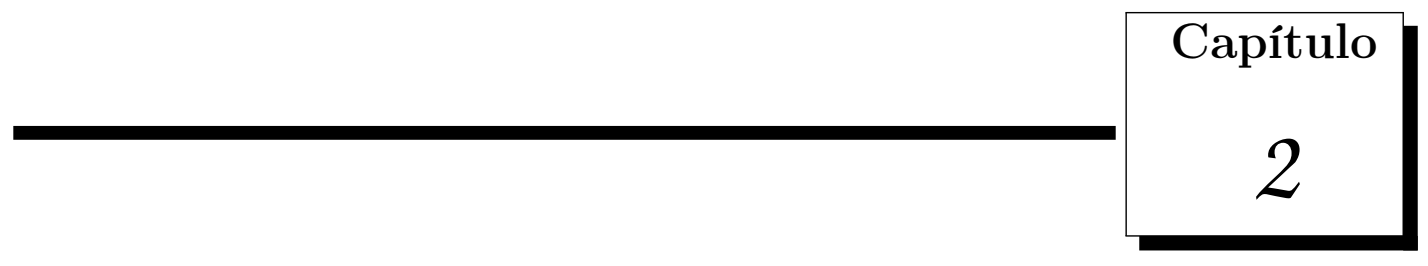

\section{Representação de aplicações de} superfícies orientáveis em $W_{n}$

2.1 Representação das classes de homotopia de aplicações de $S^{2}$ em $W_{n}$

Como na seção 1.1. nesta seção representaremos aplicações com domínio a esfera $S^{2}$, mas mudaremos o contradomínio por outro espaço.

Consideremos o espaço topológico $W_{n} \cong \underbrace{S^{2} \vee \ldots \vee S^{2}}_{n-\text { vezes }} \vee S^{1}$ dado como na ilustração abaixo:

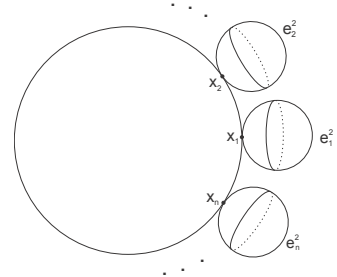

Figura 2.1: Espaço $W_{n}$

Dada uma aplicação $f: S^{2} \rightarrow W_{n}$ usaremos o seguinte diagrama comutativo 


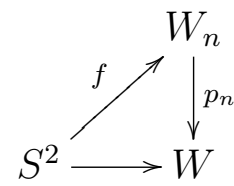

para descrever a classe de homotopia da aplicação $f$, onde $p_{n}: W_{n} \rightarrow W$ é o recobrimento de n-folhas e $W$ é como antes.

Sejam $f: S^{2} \rightarrow W_{n}$ contínua e $p_{n}: W_{n} \rightarrow W$ o recobrimento a n-folhas de $W$. Então $f$ induz uma aplicação $f^{\prime}: S^{2} \rightarrow W$ tomando a composição natural $f \circ p_{n}$. Então $f$ é o levantamento de $f^{\prime}$, isto é, o diagrama

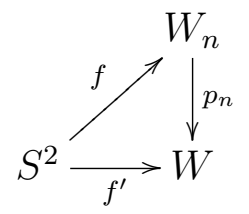

comuta.

Na seção 1.1 foram representadas as classes de homotopia de aplicações $f^{\prime}: S^{2} \rightarrow W$. Usando levantamento de homotopia podemos representar a classe da aplicação $f: S^{2} \rightarrow$ $W_{n}$.

Portanto basta descrever a aplicação $p_{n}: W_{n} \rightarrow W$ e os levantamentos das homotopias para representar $f: S^{2} \rightarrow W_{n}$.

A aplicação $p_{n}: W_{n} \rightarrow W$ é da seguinte forma:

i) Consideremos $W_{n}$ como na ilustração abaixo.

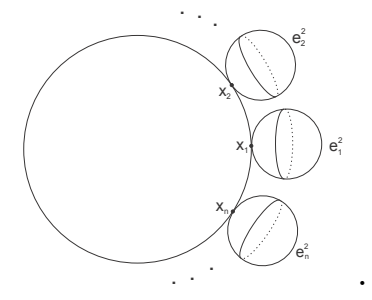

Denotemos por $e_{i}^{1} \subset W_{n} \backslash\left(\cup e_{i}^{2} \backslash\left\{x_{i}\right\}\right)$ o menor caminho que liga as 0-células $x_{i}$ a $x_{i+1}$ para $i \in\{1, \ldots, n-1\}$ e $e_{n}^{1}$ o menor caminho que liga as 0 -células $x_{n}$ a $x_{1}$.

Na ilustração o subconjunto $e_{i}^{2}$ é a esfera de dimensão dois tal que $x_{i} \in e_{i}^{2}$.

ii) observemos que $p_{n}\left(e_{i}^{1}\right)=S^{1}$ e $p_{n}\left(e_{i}^{2}\right)=S^{2}$ tal que $\left.p_{n}\right|_{\left(e_{i}^{1} \backslash\left\{x_{i}\right\}\right)}$ é injetiva em $S^{1}$ e $\left.p_{n}\right|_{e_{i}^{2}}$ é 
injetiva em $S^{2}$.

Consideremos $f^{\prime}=f \circ p_{n}: S^{2} \rightarrow W$. Pelo Teorema 1.1.1 existe uma homotopia $H: S^{2} \times I \rightarrow W \operatorname{com} H(x, 0)=f^{\prime}(x), H(x, 1)=g^{\prime}(x)$ tal que $g^{\prime}: S^{2} \rightarrow W$ é uma aplicação especial.

Como $f$ é o levantamento de $f^{\prime}$, podemos levantar a homotopia $H$ a uma homotopia $\widetilde{H}: S^{2} \times I \rightarrow W_{n} \operatorname{com} \widetilde{H}(x, 0)=f(x), \widetilde{H}(x, 1)=g(x)$ tal que $g$ é levantamento de $g^{\prime}$, isto é, $g^{\prime}=g \circ p_{n}$.

Temos o diagrama

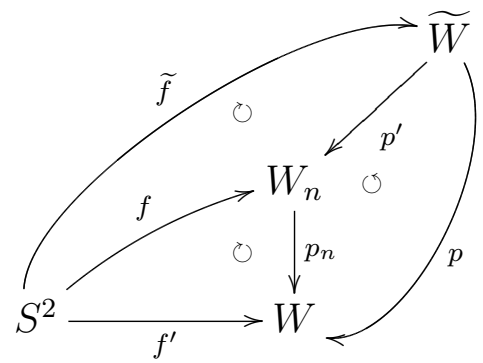

comutativo, $\tilde{f}$ é o levantamento de $f$ e o levantamento de $f^{\prime}$ ao recobrimento universal $\widetilde{W}$.

Analogamente feito na seção 1.1 podemos calcular a homologia com coeficientes locais $H_{*}\left(W_{n}, \mathbb{Z}[\mathbb{Z}]_{\rho_{\pi_{1}}}\right)$. É simples verificar que a esta homologia no nível 2 é dada por $H_{2}\left(W_{n}, \mathbb{Z}[\mathbb{Z}]_{\rho}\right) \simeq \underbrace{\mathbb{Z}[\mathbb{Z}] \oplus \ldots \oplus \mathbb{Z}[\mathbb{Z}]}_{n-\text { vezes }}$ e a homologia de $S^{2}$ no nível 2 é a mesma que na seção 1.1 , isto é, $H_{2}\left(S^{2}, \mathbb{Z}[\mathbb{Z}]_{\rho_{\pi_{1}}}\right) \simeq \mathbb{Z}[\mathbb{Z}]$.

Logo se fizermos análogo como na seção 1.1, então dada uma aplicação $f: S^{2} \rightarrow W_{n}$ associaremos uma n-upla de polinômios $\left(p_{1}(t), \ldots, p_{n}(t)\right) \simeq \underbrace{\mathbb{Z}[\mathbb{Z}] \oplus \ldots \oplus \mathbb{Z}[\mathbb{Z}]}_{n-\text { vezes }}$ ao invés de associar somente um polinômio como antes.

Cada n-upla $\left(p_{1}(t), \ldots, p_{n}(t)\right)$ tem uma interpretação geométrica no espaço $\widetilde{W}$.

Fixado um recobrimento $p^{\prime}: \widetilde{W} \rightarrow W_{n}$ de forma que a primeira esfera $S_{0}^{2} \in \widetilde{W}$ seja recoberta, pela $f$, com grau diferente de zero, isto é, o primeiro monômio de $p_{1}(t)$ é não nulo e a potência de $t$ seja 0 . Seja $p_{j}(t)=a_{0}^{(j)}+\ldots+a_{k}^{(j)} t^{k}$ a j-ésima coordenada de $\left(p_{1}(t), \ldots, p_{n}(t)\right)$, então a interpretação geométrica é que a aplicação recobre as esferas $S_{j+n i}^{2}$ com grau $a_{i}^{j}$, para $i \in\{0, \ldots, k\}$. 
Escolhemos $p: \widetilde{W} \rightarrow W$ tal que o polinômio associado a $f^{\prime}: S^{2} \rightarrow W$ tal que o primeiro monômio seja $a_{0} \neq 0$.

Como o diagrama acima é comutativo, existe uma relação entre o polinômio associado a $f$ e o polinômio associado a $f^{\prime}$. Seja $p_{\left[f^{\prime}\right]}(t)=a_{0}+\ldots+a_{m} t^{m}$ o polinômio associado a $f^{\prime}$, então $a_{j+n i_{j}}=a_{j+n i_{j}}^{(j)}$, onde $\left(p_{1}(t), \ldots, p_{n}(t)\right)$ é o polinômio associado a $f, p_{j}(t)=$ $a_{0}^{(j)}+\ldots+a_{k_{j}}^{(j)} t^{k_{j}}$ é a j-ésima coordenada, $j \in\{1, \ldots n\}$ e $i \in\left\{0, \ldots, k_{j}\right\}$.

Portanto podemos definir o polinômio $p_{[f]}$ como na seção 1.1, isto é:

Definição 2.1.1. Dados uma aplicação $f: S^{2} \rightarrow W_{n}$ e $f^{\prime}: S^{2} \rightarrow W$ como antes. O polinômio associado a classe $[f]$ é o polinômio $p_{\left[f^{\prime}\right]}(t)$.

Da mesma forma, desde que o diagrama anterior é comutativo, podemos usar o conceito de aplicação especial para a aplicação $f$ utilizando o conceito de aplicação especial da aplicação $f^{\prime}$. Segue a definição:

Definição 2.1.2. Dada uma aplicação $f: S^{2} \rightarrow W_{n}$. Dizemos que $f$ é especial se $f^{\prime}=f \circ p_{n}: S^{2} \rightarrow W$ for especial, onde $p_{n}: W_{n} \rightarrow W$ é o recobrimento a $n$-folhas de $W$.

Fixados os recobrimentos $p_{n}: W_{n} \rightarrow W$ e $p^{\prime}: \widetilde{W} \rightarrow W_{n}$ e dado um polinômio $q(t)=a_{0}+a_{1} t+\ldots+a_{j} t^{j}$, construímos, do mesmo modo da seção 1.1, a aplicação $\widetilde{f}: S^{2} \rightarrow \widetilde{W}$ e definimos $f: S^{2} \rightarrow W_{n}$ por $p^{\prime} \circ \widetilde{f}$. É fácil ver que $p_{[f]}(t)=q(t)$.

Apesar das definições acima requisitarem as definições da seção 1.1, a interpretação geométrica é diferente.

Suponha que $p_{n}$ seja tal que $p_{n}\left(S_{i}^{2}\right)=e_{i+1}^{2}$ para $i \in\{0, \ldots, n-1\}$. Consideremos os polinômios $q_{1}(t)=a_{0}$ e $q_{2}(t)=b_{0}+b_{1} t$. Neste caso a aplicação especial $f_{1}^{\prime}: S^{2} \rightarrow W$ proveniente do polinômio $q_{1}$ recobre a esfera $S^{2} \subset W$ com grau $a_{0}$ e a aplicação especial $f_{1}: S^{2} \rightarrow W_{n}$ proveniente do polinômio $q_{1}$ recobre a esfera $e_{1}^{2}$ com grau $a_{0}$.

No caso da aplicação especial $f_{2}^{\prime}: S^{2} \rightarrow W_{n}$ proveniente do polinômio $q_{2}$ existem dois discos fechados $D^{\prime 1}, D^{\prime 2} \in S^{2}$ tal que $\left.f_{2}^{\prime}\right|_{\left(D_{i}^{\prime}, \partial D_{i}^{\prime}\right)}$ recobre $S^{2} \in W$ com grau $a_{i}$ e $\left.f_{2}^{\prime}\right|_{S^{2} \backslash\left(D_{1}^{\prime} \cup D^{\prime 2}\right)}$ recobre $W \backslash S^{2}$ e a aplicação especial $f_{2}: S^{2} \rightarrow W_{n}$ existem dois discos $D_{1}, D_{2} \in S^{2}$ tal que $\left.f_{2}\right|_{\left(D_{i}^{\prime}, \partial D_{i}^{\prime}\right)}$ recobre $e_{i}^{2} \in W_{n}$ com grau $a_{i}$ e $\left.f_{2}\right|_{S^{2} \backslash\left(D_{1} \cup D^{2}\right)}$ recobre $e_{1}^{1}$.

Teorema 2.1.1. Seja uma aplicação $f: S^{2} \rightarrow W_{n}$. Então existe aplicação $g \in[f]$ especial. 
Demonstração: Dadas uma aplicação $f: S^{2} \rightarrow W_{n}$ e o recobrimento de n- folhas $p_{n}: W_{n} \rightarrow W$. Consideremos a aplicação $f^{\prime}=p_{n} \circ f$.

Pelo Teorema 1.1 .1 existe $g^{\prime} \in\left[f^{\prime}\right]$ especial. Seja $\widetilde{g}^{\prime}: S^{2} \rightarrow \widetilde{W}$ o levantamento de $g^{\prime}$ construído a partir do polinômio $p_{\left[g^{\prime}\right]}(t)$.

Basta tomar $g=p \circ \widetilde{g}^{\prime}$, onde $p^{\prime}: \widetilde{W} \rightarrow W_{n}$ é o recobrimento universal.

\subsection{Representação das classes de homotopia de aplica- ções de $T^{2}$ em $W_{n}$}

Consideremos o espaço topológico $W_{n} \cong \underbrace{S^{2} \vee \ldots \vee S^{2}}_{n-\text { vezes }} \vee S^{1}$ dado como na ilustração 2.1 e o espaço $W$ com antes.

Dada uma aplicação $f: T^{2} \rightarrow W_{n}$, consideremos o seguinte diagrama comutativo

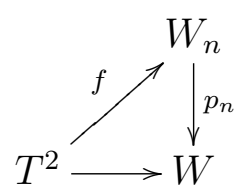

onde $p_{n}: W_{n} \rightarrow W$ é o recobrimento de n-folhas de $W$, representada como na seção 2.2.

Portanto $f$ induz uma aplicação $f^{\prime}: T^{2} \rightarrow W$ tomando a composição natural $f \circ p_{n} \mathrm{e}$ $f$ é o levantamento de $f^{\prime}$, isto é, o diagrama

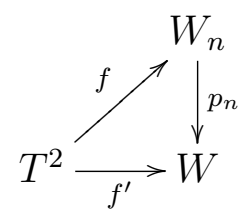

comuta.

Pela seção 1.2 temos a representação da classe de homotopia da aplicação $f^{\prime}: T^{2} \rightarrow W$. Usando levantamento de homotopia podemos representar as classes da aplicação $f: T^{2} \rightarrow$ $W_{n}$.

Portanto basta descrever a aplicação $p_{n}: W_{n} \rightarrow W$ e usar levantamentos das homoto- 
pias para representar $f: S^{2} \rightarrow W_{n}$

Usamos o diagrama comutativo acima para definir o polinômio associado a classe de homotopia $[f]$, como segue:

Definição 2.2.1. Sejam uma aplicaçãoo $f: T^{2} \rightarrow W$ e o polinômio $p_{\left[f^{\prime}\right]}(t)$. Então $p_{[f]}(t)=$ $p_{\left[f^{\prime}\right]}(t)$ é o polinômio associado a $[f]$.

\section{Construção de $f: T^{2} \rightarrow W_{n}$ a partir de $q(t)$ e $l \in \mathbb{N}$}

Dados um polinômio $q(t)=a_{0}+\ldots+a^{j} t^{j}$ e um número $l \in \mathbb{N}$. Considere a aplicação $f$ de $T^{2}$ em $W_{n}$ obtida da figura

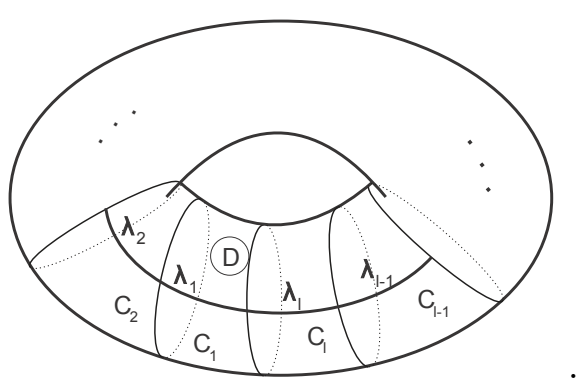

onde $f(\partial D)=P,\left.f\right|_{(D, \partial D)}:(D, \partial D) \rightarrow W$ é construída a partir do polinômio $q(t)$ como na seção 2.1] (como aplicação de $S^{2}$ em $W_{n}$ ) e a imagem dos cilindros $C_{i}$ é $W_{n} \backslash \bigsqcup_{k=1}^{l} e_{k}^{2} \backslash\{k\}$ tal que cada um dos caminhos $\lambda_{i}$ é levado pela $f$ em $S^{1} \subset W$ no sentido antihorário e $i \in\{1, \ldots, l\}$.

Quando $l=0$, então $f\left(T^{2} \backslash \stackrel{\circ}{D}\right)=P,\left.f\right|_{(D, \partial D)}:(D, \partial D) \rightarrow W$ é construída a partir do polinômio $q(t)$ como na seção 2.1. Neste caso é equivalente (a menos de homotopia) a aplicação tal que $f\left(S^{1} \vee S^{1}\right)=P$ e $\left.f\right|_{T^{2} \backslash S^{1} \vee S^{1}}$ é construída a partir do polinômio $q(t)$.

Portanto por construção $p_{[f]}(t)=q(t)$ e desde que $\pi_{1}\left(T^{2}\right) \simeq \mathbb{Z} \oplus \mathbb{Z}=\langle\alpha\rangle \oplus<\beta>$, então $f_{\pi}\left(\pi_{1}\left(T^{2}\right)\right)=l \mathbb{Z}$, onde $f_{\pi}(\alpha)=l$ e $f_{\pi}(\beta)=0$.

Definição 2.2.2. Uma aplicação $f: T^{2} \rightarrow W_{n}$ será chamada especial se ela pode ser construída como acima, isto é, a partir do polinômio $p_{[f]}(t)$ e de $l$, onde $l \in \mathbb{N}$ é um gerador de $f_{\pi}\left(\pi_{1}\left(T^{2}\right)\right)$ ou $l=0$. 
Observe que se $f: T^{2} \rightarrow W_{n}$ é especial construída a partir de $p_{[f]}(t)$ e $l \in \mathbb{N}$, então $\left(p_{n}\right)_{\pi}(l)=n l \in \pi_{1}(W)$ e $f^{\prime}: T^{2} \rightarrow W$ é especial construída a partir de $p_{[f]}(t)$ e $n l \in \mathbb{N}$.

Representação de $f: T^{2} \rightarrow W_{n}$

Dada uma aplicação $f: T^{2} \rightarrow W_{n}$, dividiremos em casos para provar a existência de uma aplicação especial $g \in[f]$.

Primeiro Caso: Suponha que $f_{\pi}=0$.

Neste caso temos o seguinte diagrama comutativo

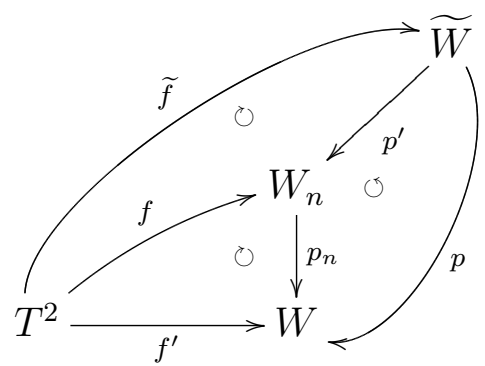

Portanto, $f$ é especial se, e somente se, $f^{\prime}$ é especial. Pelo Teorema 1.2.1 existe $g^{\prime} \in\left[f^{\prime}\right]$ especial. Logo, utilizando levantamento de homotopias, existe $g \in[f]$ especial.

Observemos que a aplicação especial $f: T^{2} \rightarrow W_{n}$ pode não ser sobrejetora, mas aplicação especial $f^{\prime}: T^{2} \rightarrow W$ ser sobrejetora. Isto acontece quando $p_{[f]}(t)=a_{0}+a_{1} t+$ $\ldots+a_{j} t^{j}$, onde $a_{0} \neq 0, a_{j} \neq 0$ para algum $0<j<n$.

Segundo Caso: Suponha que $f_{\pi} \neq 0$.

Seja $f: T^{2} \rightarrow W_{n}$ tal que $f_{\pi} \neq 0$. Identificando $\pi_{1}\left(W_{n}\right) \operatorname{com} \mathbb{Z}$, temos $f_{\pi}\left(\pi_{1}\left(T^{2}\right)\right) \simeq<$ $l>\simeq \mathbb{Z}$, onde $0 \neq l \in \mathbb{N}$.

Da maneira análoga da seção 1.2 podemos supor que os geradores $\alpha, \beta \in \pi_{1}\left(T^{2}\right)$ são tal que $f_{\pi}(\alpha)=l$ e $f_{\pi}(\beta)=0$. Assim levantamos $f$ ao $\bar{T}$ recobrimento cíclico infinito de $T^{2}$ relativo ao $\operatorname{ker}\left(f_{\pi}\right)$ e temos o diagrama comutativo

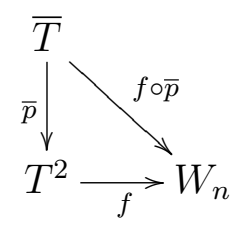


Por construção do recobrimento $\bar{T}$, a imagem $(f \circ \bar{p})_{\pi}\left(\pi_{1}(\bar{T})\right)$ é nula. Portanto a aplicação $f \circ \bar{p}$ se levanta ao recobrimento universal $\widetilde{W}$, isto é, o diagrama

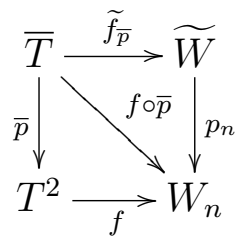

comuta.

Portanto temos o seguinte diagrama comutativo:

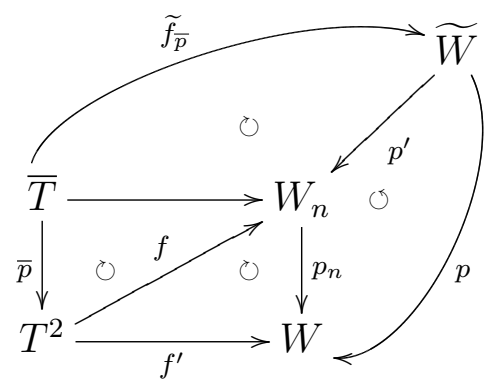

Consideremos $f^{\prime}=f \circ p_{n}: T^{2} \rightarrow W$. Pelo Teorema 1.2.1 existe uma homotopia $H: T^{2} \times I \rightarrow W \operatorname{com} H(x, 0)=f^{\prime}(x), H(x, 1)=g^{\prime}(x)$ tal que $g^{\prime}: T^{2} \rightarrow W$ é uma aplicação especial.

A aplicação especial $g^{\prime}$ pode ser construída. A construção é feita por modificações, através dos Lemas 1.2.1, 1.2.2 e algumas técnicas geométricas, na região $C \subset \bar{T}$ para encontrar uma aplicação $\widetilde{g}_{\bar{p}}: \bar{T} \rightarrow \widetilde{W}$, tal que $\widetilde{g}_{\bar{p}} \in\left[\widetilde{f}_{\bar{p}}\right]$ e a partir da aplicação $\bar{p}$ são feitas modificações em $T^{2}$ e compondo com a aplicação $p$ teremos a aplicação especial $g^{\prime}$.

Podemos repetir este processo e as técnicas usadas na seçao 1.2 e utilizando o diagrama 2.2 e a aplicação $p_{n}$ encontramos uma aplicação especial $g: T^{2} \rightarrow W_{n}$ tal que $g \in[f]$.

Portanto temos o seguinte teorema:

Teorema 2.2.1. Seja uma aplicação $f: T^{2} \rightarrow W_{n}$. Então existe aplicação $g \in[f]$ é especial. 


\subsection{Representação das classes de homotopia de aplica-}

\section{ções de $T_{g}$ em $W_{n}$}

Sejam $T_{g}$ uma superfície fechada, conexa e orientável de gênus $g>1$ e uma aplicação $f: T_{g} \rightarrow W_{n}$.

Associaremos um polinômio a $[f]$ e a partir deste polinômio encontraremos um elemento $g \in[f]$ com boas propriedades.

\section{Polinômio associado a uma aplicação de $T_{g}$ em $W_{n}$}

O grupo fundamental de uma superfície $T_{g}$ fechada e orientável de gênus $g>1$ é dado por

$$
\pi_{1}\left(T_{g}\right)=<\alpha_{1}, \beta_{1}, \alpha_{2}, \beta_{2}, \ldots, \alpha_{g}, \beta_{g} ; \alpha_{1} \beta_{1} \alpha_{1}^{-1} \beta_{1}^{-1} \alpha_{2} \beta_{2} \alpha_{2}^{-1} \beta_{2}^{-1} \ldots \alpha_{g} \beta_{g} \alpha_{g}^{-1} \beta_{g}^{-1}>
$$

De maneira análoga como foi feito para associar um polinômio a uma aplicação de $T^{g}$ em $W$, podemos escolher convenientemente os geradores de $\pi_{1}\left(T_{g}\right)$ tal que $\alpha_{1}$ pertence a imagem inversa $f^{-1}(x)$, onde $x$ é valor regular de $f$. A superfície pode ser obtida de polígono identificando os lados que são os geradores de $\pi_{1}\left(T_{g}\right)$ como ilustrado antes.

Quando $f_{\pi}=0$, então $f_{\pi}\left(\alpha_{i}\right)=f_{\pi}\left(\beta_{i}\right)=0$ para todo $i \in\{1, \ldots, g\}$. Caso $f_{\pi} \neq 0$, existe $l \in \mathbb{N}^{*}$ tal que $f_{\pi}\left(\pi_{1}\left(T_{g}\right)\right) \simeq \mathbb{Z}<l>\subset \mathbb{Z} \simeq \pi_{1}(W)$.

Assim como foi feito no caso do toro, podemos considerar os geradores de $\pi_{1}\left(T_{g}\right)$ satisfazendo $f_{\pi}\left(\alpha_{1}\right)=l$ e $f_{\pi}\left(\alpha_{i}\right)=f_{\pi}\left(\beta_{j}\right)=0$, onde $i \in\{2, \ldots, g\}$ e $j \in\{1, \ldots, g\}$.

Dada uma aplicação $f: T^{g} \rightarrow W_{n}$, consideremos o seguinte diagrama comutativo

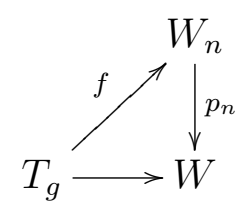

onde $p_{n}: W_{n} \rightarrow W$ é o recobrimento de n-folhas de $W$.

Definição 2.3.1. O polinômio $p_{\left[f^{\prime}\right](t)}$ será o polinômio associado à $[f]$ e denotaremos 
$p_{[f]}(t)$.

\section{Construção de uma aplicação a partir de um $p(t)$ e $l \in \mathbb{N}$}

Dada uma superfície fechada e orientável $T_{g}$ de gênus $g \geq 1$, então pelo teorema de classificação de superfície $T_{g} \simeq \underbrace{T^{2} \# \ldots \# T^{2}}_{g \text {-vezes }}$, onde \# é a colagem pelo bordo de um disco removendo o seu interior.

Portanto o recobrimento cíclico infinito $\overline{T_{g}}$ de uma superfície $T_{g}$ é como segue

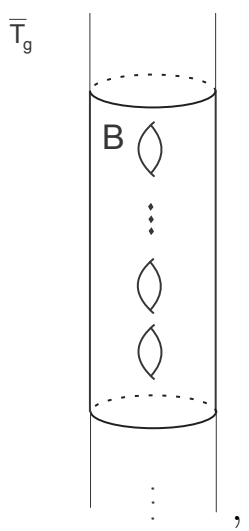

onde a região destacada é uma região básica e será denotado por $B$.

Para definir uma aplicação $\bar{f}_{\bar{p}}: \overline{T_{g}} \rightarrow \widetilde{W}$ basta defini-lá somente na região $B$ e depois estender analogamente a definição nas outras cópias de $B$, por isso $B$ é chamada de região fundamental.

Sejam um polinômio $q(t)=a_{0}+\ldots+a^{j} t^{j}$ e um número $l \in \mathbb{N}$.

Considere a aplicação $\bar{f}_{\bar{p}}$ definida na região $B$ da seguinte maneira 


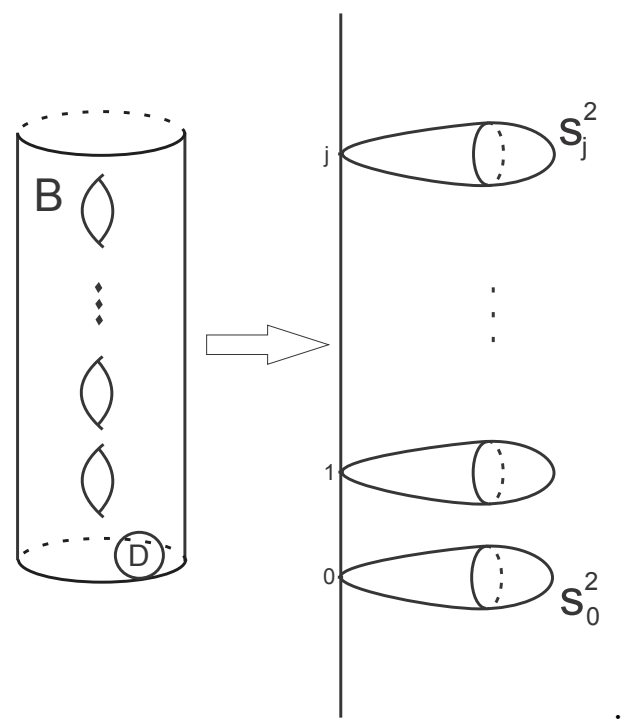

onde $\bar{f}_{\bar{p}}(\partial D)=\{P\},\left.\bar{f}_{\bar{p}}\right|_{(D, \partial D)}:(D, \partial D) \rightarrow \widetilde{W}$ é construída a partir do polinômio $q(t)$ como na seção 1.1 e $\bar{f}_{B \backslash D}$ é sobrejetiva no intervalo $[0, \ln ]$.

Por fim, definimos a aplicação $f: T_{g} \rightarrow W_{n}$ por

$$
f(x)=p^{\prime} \circ \bar{f}_{\bar{p}}(\bar{x})
$$

onde $p^{\prime}: \widetilde{W} \rightarrow W_{n}$ é aplicação de recobrimento universal, $\bar{p}: \overline{T_{g}} \rightarrow T_{g}$ é aplicação recobrimento e $\bar{x}=\bar{p}^{-1}(x) \cap B$.

Portanto por construção $p_{[f]}(t)=q(t)$ e $f_{\pi}\left(\pi_{1}\left(T_{g}\right)\right)=<l>\subset \mathbb{Z} \simeq \pi_{1}(W)$, onde $f_{\pi}\left(\alpha_{1}\right)=l$ e $f_{\pi}\left(\alpha_{i}\right) f_{\pi}\left(\beta_{j}\right)=0$ para $i \in\{2, \ldots, g\}$ e $j \in\{1, \ldots, g\}$.

Definição 2.3.2. Uma aplicação $f: T_{g} \rightarrow W_{n}$ será chamada especial se ela pode ser construída como acima, isto é, a partir do polinômio $p_{[f]}(t)$ e de $l$, onde $l \in \mathbb{N}$ é um gerador de $f_{\pi}\left(\pi_{1}\left(T_{g}\right)\right)$.

\section{Encontrando uma aplicação especial $g \in[f]$}

Dada uma aplicação $f: T_{g} \rightarrow W_{n}$, dividiremos em dois casos para provar a existência de uma aplicação especial $g \in[f]$.

Os dois casos serão como foi feito em aplicações de $T^{2}$ em $W_{n}$.

Primeiro Caso: Suponha que $f_{\pi}=0$. 
Tomamos o seguinte diagrama comutativo

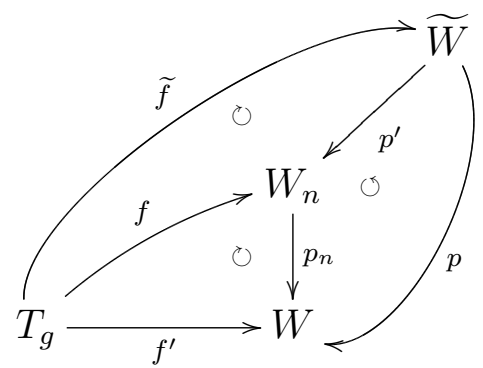

Analogamente o caso de aplicações de $T^{2}$ em $W^{n}$, prova-se que existe $g \in[f]$ aplicação especial.

Segundo Caso: Suponha que $f_{\pi} \neq 0$.

Seja $f: T_{g} \rightarrow W_{n}$ tal que $f_{\pi} \neq 0$. Identificando $\pi_{1}\left(W_{n}\right)$ com $\mathbb{Z}$, temos $f_{\pi}\left(\pi_{1}\left(T_{g}\right)\right) \simeq$ $\mathbb{Z}<l>\subset \mathbb{Z} \simeq \pi_{1}\left(W_{n}\right)$, onde $0 \neq l \in \mathbb{N}$.

Da maneira análoga da seção 1.2 podemos supor que os geradores $\alpha_{i} \in \pi_{1}\left(T_{g}\right)$ são tal que $f_{\pi}\left(\alpha_{0}\right)=l$ e $f_{\pi}\left(\alpha_{i}\right)=0$ para $i \neq 0$. Assim levantamos $f$ ao $\overline{T_{g}}$ recobrimento cíclico infinito de $T_{g}$ relativo ao $\operatorname{ker}\left(f_{\pi}\right)$ e temos o diagrama comutativo

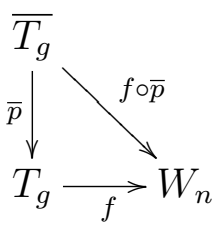

Por construção do recobrimento $\overline{T_{g}}$, a imagem $(f \circ \bar{p})_{\pi}\left(\pi_{1}\left(\overline{T_{g}}\right)\right)$ é nula. Portanto a aplicação $f \circ \bar{p}$ se levanta ao recobrimento universal $\widetilde{W}$, isto é, o diagrama

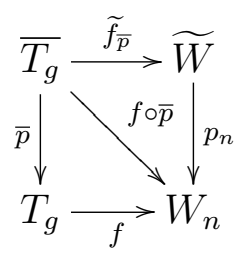

comuta.

Portanto temos seguinte diagrama: 


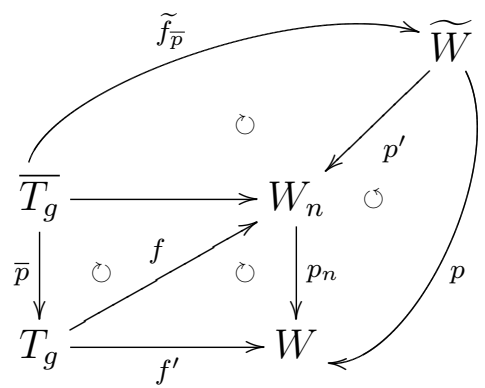

Pelo Teorema 1.3 .1 existe uma homotopia $H: S^{2} \times I \rightarrow W \operatorname{com} H(x, 0)=f^{\prime}(x)$, $H(x, 1)=g^{\prime}(x)$ tal que $g^{\prime}: T^{2} \rightarrow W$ é uma aplicação especial.

A aplicação especial $g^{\prime}$ pode ser construída. A construção é feita por modificações, através dos Lemas 1.3.1, 1.3.2 e algumas técnicas geométricas, na região $B \subset \overline{T_{g}}$ para encontrar uma aplicação $\widetilde{g}_{\bar{p}}: \overline{T_{g}} \rightarrow \widetilde{W}$, tal que $\widetilde{g}_{\bar{p}} \in\left[\widetilde{f}_{\bar{p}}\right]$ e a partir da aplicação $\bar{p}$ são feitas modificações em $T^{2}$ e compondo com a aplicação $p$ teremos a aplicação especial $g^{\prime}$.

Podemos repetir este processo e as técnicas usadas na seção 1.3 e utilizando o diagrama acima e compondo com a aplicação $p_{n}$ encontramos uma aplicação especial $g: T_{g} \rightarrow W_{n}$ tal que $g \in[f]$.

Portanto temos o seguinte teorema:

Teorema 2.3.1. Seja $f: T_{g} \rightarrow W_{n}$ uma aplicação. Então existe aplicação $g \in[f]$ especial. 


\section{Capítulo}

\section{Representação de aplicações de}

\section{superfícies não orientáveis em $W$}

Nas próximas duas seções, assim como na seção anterior, dada uma aplicação $f$ : $\mathbb{R} P_{g}^{2} \rightarrow W$ é associado um polinômio $p_{[f]}(t)$ a classe de homotopia de $f$, mas este polinômio tem coeficientes em $\mathbb{Z}_{2}$. Apresenta-se uma aplicação, chamada aplicação especial, a qual no capítulo de ?? mostra-se que estas aplicações realizam o conjunto minimal de raízes, que é construída a partir de um polinômio. Depois, mostra-se que existe uma aplicação especial em cada classe de homotopia $\left[\mathbb{R} P_{g}^{2}, W\right]$.

O motivo de considerar polinômios com coeficientes em $\mathbb{Z}_{2}$ é explicado no apêndice.

\subsection{Representação das classes de homotopia de aplica- ções de $\mathbb{R} P^{2}$ em $W$}

Consideremos o espaço projetivo $\mathbb{R} P^{2}$ e uma aplicação $f: \mathbb{R} P^{2} \rightarrow W$. Então temos o seguinte diagrama comutativo 


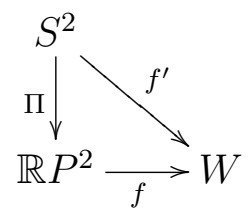

onde $\Pi: S^{2} \rightarrow \mathbb{R} P^{2}$ é o recobrimento duplo orientável.

Como $\pi_{1}\left(\mathbb{R} P^{2}\right) \simeq \mathbb{Z}_{2}$, segue que $f_{\pi}=0$. Logo o diagrama

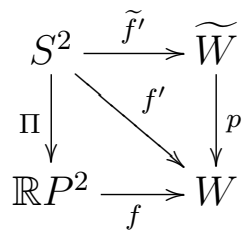

comuta.

Para fazer modificações, por homotopias, na aplicação $f$ basta fazer modificações numa região fundamental de $S^{2}$ e repetir nas outras regiões e utilizando a aplicação $\Pi$ para modificar $f$. Neste caso como $\Pi$ é recobrimento duplo a região que vamos considerar é a calota superior de $S^{2}$, isto é, se $S^{2}=\left\{(x, y, z) \in \mathbb{R}^{3}:|x|^{2}+|y|^{2}+|z|^{2}=1\right\}$, então a região será $C N=\left\{(x, y, z) \in \mathbb{R}^{3}:|x|^{2}+|y|^{2}+|z|^{2}=1\right.$ e $\left.z \geq 0\right\} \subset S^{2}$.

Sem perda de generalidade podemos supor que $f \circ \Pi\left(S^{1}\right)=P$, onde $S^{1}=\{(x, y, z) \in$ $\mathbb{R}^{3}:|x|^{2}+|y|^{2}+|z|^{2}=1$ e $\left.z=0\right\} \subset S^{2}$.

Identificamos $C N \backslash S^{1} \operatorname{com} S^{2} \backslash N$, para algum $N \in S^{2}$. Consideremos a aplicação $h: S^{2} \rightarrow W$ definida por

$$
h(x)=\left\{\begin{array}{lll}
f^{\prime}(x) & \text { se } & x \in S^{2} \backslash N \\
P & \text { se } & x=N
\end{array}\right.
$$

e seu polinômio $p_{[h]}(t)=a_{0}+a_{1} t+\ldots+a_{n} t^{n}$. Seja $\bar{p}_{[h]}(t)=\bar{a}_{0}+\bar{a}_{1} t+\ldots+\bar{a}_{n} t^{n}$ o polinômio tal que $\bar{a}_{i}$ é igual a $a_{i}$ módulo 2 .

Definição 3.1.1. Dada uma aplicação $f: \mathbb{R} P^{2} \rightarrow W$, definimos o polinômio associado a classe de homotopia $[f]$ por $\bar{p}_{[h]}(t)$, onde $h: S^{2} \rightarrow W$ é como antes e este polinômio será denotado por $p_{[f]}(t)$.

Agora a partir de um polinômio qualquer $q(t)$ com coeficientes em $\mathbb{Z}_{2}$, construiremos 
uma aplicação $f: \mathbb{R} P^{2} \rightarrow W$ tal que $p_{[f]}(t)=q(t)$.

Dado um polinômio $q(t)=a_{0}+\ldots+a_{n} t^{n}$. Consideremos a aplicação $h: S^{2} \rightarrow W$ construída a partir de $q(t)$, como feito na seção 1.1 .

Identificamos a região $C N \backslash S^{1} \operatorname{com} S^{2} \backslash N$, tal que $h(N)=P$.

Dado $y \in \mathbb{R} P^{2}$, então $\Pi^{-1}(y)=\{x,-x\}$ tal que $x \in C N \backslash S^{1}$ e $-x \in \mathbb{R} P^{2} \backslash C N$ ou $\Pi^{-1}(y) \subset S^{1}$.

Definimos $f: \mathbb{R} P^{2} \rightarrow W$ por

$$
f(x)=\left\{\begin{array}{lll}
h(x) & \text { se } & x \in C N \backslash S^{1} \\
P & \text { se } & \Pi^{-1}(y) \subset S^{1}
\end{array}\right.
$$

Por construção $p_{[f]}(t)=q(t)$.

Definição 3.1.2. Uma aplicação $f: \mathbb{R} P^{2} \rightarrow W$ que pode ser construída como acima é chamada aplicação especial.

Dada uma classe de homotopia $[f]$ de uma aplicação $f: \mathbb{R} P^{2} \rightarrow W$, mostraremos que existe $g \in[f]$ tal que $g$ é uma aplicação especial.

Seja uma homotopia $F: S^{2} \times I \rightarrow W$ entre duas aplicações $f^{\prime}, g^{\prime}: S^{2} \rightarrow W$ tal que as modificações feitas por $F$ em $C N \times I$ se repete na região $\left(S^{2} \backslash C N\right) \times I$. Então utilizando a aplicação $\Pi$ induzimos uma homotopia $\bar{F}: \mathbb{R} P^{2} \times I \rightarrow W$ entre duas $f, g: \mathbb{R} P^{2} \rightarrow W$ tais que $f \circ \Pi=f^{\prime}$ e $g \circ \Pi=g^{\prime}$.

Consideremos uma aplicação $f: \mathbb{R} P^{2} \rightarrow W$ e a aplicação $h: S^{2} \rightarrow W$ definida como antes a partir de $f$. Então pelo Teorema 1.1.1 existe uma aplicação especial $h^{\prime} \in[h]$.

Identificamos o conjunto $C N$ com $S^{2} \backslash N$, onde $h(N)=h^{\prime}(N)=P$. Definimos duas aplicações $f^{\prime}, g^{\prime}: S^{2} \rightarrow W$ por $f^{\prime}(x)=f^{\prime}(-x)=h(x)$ e $g^{\prime}(x)=g^{\prime}(-x)=h^{\prime}(x)$, onde $x \in$ $C N$ e $-x \in S^{2} \backslash C N$. Dada uma homotopia entre $h^{\prime}$ e $h$, podemos induzir uma homotopia entre $f^{\prime}$ e $g^{\prime}$. Portanto podemos induzir uma homotopia $\bar{F}$ tal que $\bar{F}(x, 0)=f(x)$ e $g(x)=\bar{F}(x, 1)$ tal que $g$ é especial.

Logo segue o teorema:

Teorema 3.1.1. Seja $f: \mathbb{R} P^{2} \rightarrow W$ uma aplicação. Então existe $g \in[f]$ tal que g é uma aplicação especial. 
Observe que o polinômio associado a cada classe de homotopia de uma aplicação especial tem coeficientes em $\mathbb{Z}_{2}$. E acima concluímos que $g$ é especial. Isto se deve ao fato de que $h$ construída a partir de $f$, como acima, possui a propriedade de que o polinômio associado a $[h]$ tem coeficientes em $\mathbb{Z}_{2}$. No apêndice este fato fica esclarecido.

\subsection{Representação das classes de homotopia de aplica- ções de $\mathbb{R} P_{g}^{2}$ em $W$}

Nesta seção representaremos classes de homotopias de aplicações de uma superfície compacta e não orientável em $W$.

Pelo Teorema de Classificação de Superfícies uma superfície não orientável é dada por

$$
\underbrace{\mathbb{R} P^{2} \# \ldots \# \mathbb{R} P^{2}}_{g \text {-vezes }}
$$

isto é, a soma conexa de $g$ planos projetivos, para algum $g$ finito.

Denotaremos a soma conexa de $g$ planos projetivos por $\mathbb{R} P_{g}^{2}$.

No caso de $g=1$ está resolvido, por isso consideremos $g \geq 2$.

Assim como na seção anterior, usaremos o recobrimento duplo orientável de $\mathbb{R} P_{g}^{2}$ para representar as classes de homotopia de aplicações $f: \mathbb{R} P_{g}^{2} \rightarrow W$.

Temos as seguintes identificações;

- $\mathbb{R} P_{2}^{2} \cong K$, onde $K$ é a garrafa de Klein,

- $\mathbb{R} P_{g}^{2} \cong T_{k}^{2} \# \mathbb{R} P^{2}$, onde $g=2 k+1 \mathrm{e}$

- $\mathbb{R} P_{g}^{2} \cong T_{k}^{2} \# K$, onde $k \neq 2$ e $g=2 k$.

No caso da garrafa de Klein o recobrimento duplo orientável é o toro, como na figura abaixo:

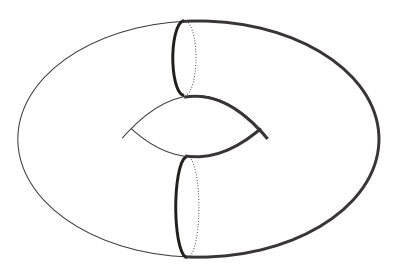

Figura 3.1: Recobrimento duplo da garrafa de Klein 
onde a parte destacada é uma região fundamental.

No segundo caso o recobrimento duplo orientável é um cilindro tal que em cada extremidade do cilindro é feita a soma conexa com $T_{k}^{2}$ como na figura abaixo

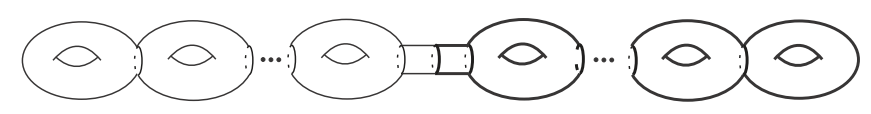

Figura 3.2: $g$ impar

onde o cilindro central recobre $\mathbb{R} P^{2} \backslash D$, onde $D$ é um disco e a parte destacada da figura é uma região fundamental.

No terceiro caso o recobrimento duplo orientável é dado tomando um toro e fazendo soma conexa de dois $T_{k}^{2}$ como na ilustração abaixo

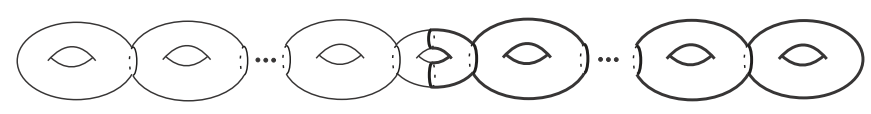

Figura 3.3: $g$ par

onde o toro central recobre a garrafa de Klein e a região destacada é uma região fundamental.

Portanto em cada caso temos um diagrama comutativo como abaixo

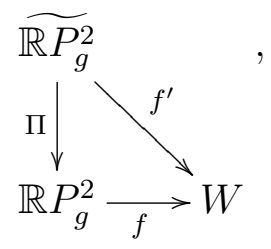

onde $\widetilde{\mathbb{R} P_{g}^{2}}$ é o recobrimento duplo orientável de $\mathbb{R} P_{g}^{2}$.

O resultado do Lema 5.3 de [6] também é válido para uma aplicação $f: \mathbb{R} P_{g}^{2} \rightarrow W$. Logo para variedades não orientável podemos utilizar das técnicas feita nos Lemas 1.2.1 e 1.2.2 para que $f^{-1}\left(W \backslash S^{1}\right)$ seja um disco.

Usando o levantamento de homotopia, podemos fazer estas modificações em cada região do recobrimento duplo orientável de $\mathbb{R} P_{g}^{2}$. Quando fazemos estas modificações nos levantamentos resulta em dois discos, um disco numa região fundamental e outro disco na outra região, sendo cada um destes é levado no disco de $\mathbb{R} P_{g}^{2}$ pela aplicação $\Pi$. 
Logo temos que tomar $\frac{p_{\left[f^{\prime}\right]}(t)}{2}$.

Além disso, a menos de homotopia, o polinômio $\frac{p_{\left[f^{\prime}\right]}(t)}{2}$ tem coeficientes em $\mathbb{Z}_{2}$. Este fato é esclarecido no apêndice.

Denotaremos $\bar{p}_{\left[f^{\prime}\right]}=\bar{a}_{0}+\bar{a}_{1} t+\ldots+\bar{a}_{n} t^{n}$ o polinômio tal que $\bar{a}_{i}$ são os coeficientes de $\frac{p_{\left[f^{\prime}\right]}(t)}{2}=a_{0}+a_{1} \ldots+a_{n} t^{n}$ módulo 2 .

Assim temos a definição:

Definição 3.2.1. Sejam uma aplicação $f: \mathbb{R} P_{g}^{2} \rightarrow W$ e $f^{\prime}: \widetilde{\mathbb{R} P_{g}^{2}} \rightarrow W$ tal que $f \circ \Pi=f^{\prime}$, como no diagrama acima. Então o polinômio associado a aplicação $f$ é $\bar{p}_{\left[f^{\prime}\right]}(t)$ e será denotado por $p_{[f]}(t)$.

\section{Aplicação especial de $\mathbb{R} P_{g}^{2}$ em $W$ para $g=2 k+1$}

Dados um polinômio $q(t)=a_{0}+\ldots+a_{n} t^{n}$ tal que $a_{0}=1, a_{i} \in \mathbb{Z}_{2}$ e um número $l \in \mathbb{N}$ finito. Consideremos a variedade não orientável $\mathbb{R} P_{g}^{2}$ tal que $g=2 k+1$ para algum $k \in \mathbb{N}$.

Construímos uma aplicação $f: T_{k}^{2} \rightarrow W$ a partir de $q(t)$ e $l$, como feito na seção 1.2 .

Sem perda de generalidade podemos supor que existe um disco fechado $D \subset T_{g}^{2}$ tal que $f(D)=P$. Identificamos o subconjunto $C=T_{g}^{2} \backslash \stackrel{\circ}{D}$ com uma região básica do recobrimento duplo orientável de $\mathbb{R} P_{g}^{2}$.

Definimos a aplicação $h: \mathbb{R} P_{g}^{2} \rightarrow W$ por $h([x])=f(x)$, onde $\Pi^{-1}([x]) \cap C=x$.

Por construção temos $h_{\pi}\left(\pi_{1}\left(\mathbb{R} P_{g}^{2}\right)\right) \simeq \mathbb{Z}<l>$ e $p_{[h]}(t)=q(t)$.

Definição 3.2.2. Uma aplicação que é como a aplicação h construída acima, isto é, uma aplicação que pode ser construída a partir de l finito e seu polinômio associado $p_{[h]}(t)$ é chamada aplicação especial.

Uma aplicação $h: \mathbb{R} P_{g}^{2} \rightarrow W$ é especial desde que a aplicação $f: T_{k}^{2} \rightarrow W$ construída a partir de $h$ seja uma aplicação especial. Lembrando que aplicações especiais $f$ construída a partir de $h$ tem os coeficientes do polinômio $p_{[f]}(t)$ associado em $\mathbb{Z}_{2}$ (este fato é esclarecido no apêndice). Portanto segue o teorema. 
Teorema 3.2.1. Seja uma aplicação $h: \mathbb{R} P_{g}^{2} \rightarrow W$. Então existe uma aplicação $g \in[h]$ tal que g é uma aplicação especial.

\section{Aplicação especial de $\mathbb{R} P_{g}^{2}$ em $W$ para $g=2 k$}

Primeiramente consideremos a variedade não orientável $\mathbb{R} P_{2}^{2} \cong K$, onde $K$ é a garrafa de Klein.

Dados o toro $T^{2}$ recobrimento duplo de $K$ e um polinômio $q(t)=a_{0}+\ldots+a_{n} t^{n}$ tal que $a_{0} \neq 0, a_{i} \in \mathbb{Z}_{2}$ e um número $l \in \mathbb{N}$ finito.

Consideremos a região fundamental $F$ destacada na figura 3.2. Definimos uma aplicação em $F$ da seguinte maneira:

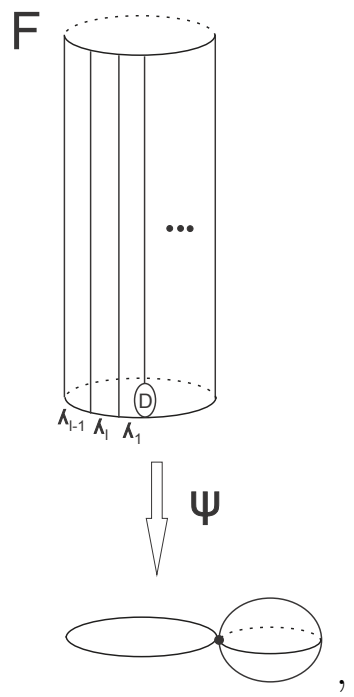

onde a aplicação é definida no disco $D$ a partir do polinômio $q(t)$, como na seção 1.1, cada faixa $\lambda_{i}$ é projetada em $S^{1} \subset W$ e o bordo do disco $D$ e os bordos das faixas são levados em $P \in W$. Denotaremos esta aplicação por $\Psi: F \rightarrow W$.

Podemos identificar $T^{2} \backslash F \operatorname{com} F \backslash \partial F$.

Definimos uma aplicação $h: T^{2} \rightarrow W$ dada por $h(x)=\Psi(x)$, isto é, a aplicação $h$ é definida como $\Psi$ em $F$. Como $T^{2} \backslash \stackrel{\circ}{F}$ também é um cilindro $h$ é definida de modo análogo a $\Psi$ em $F$.

Por fim definimos uma aplicação $f: K \rightarrow W$ por $f([x])=h(x)$, onde $\Pi^{-1}([x]) \cap F=x$.

Por construção temos $f_{\pi}\left(\pi_{1}(K)\right) \simeq \mathbb{Z}<l>$ e $p_{[f]}(t)=q(t)$. 
Definição 3.2.3. Uma aplicação $f: K \rightarrow W$ é chamada aplicação especial se pode ser construída, como acima, a partir de l finito e seu polinômio associado $p_{[f]}(t)$.

Dada uma aplicação $f: K \rightarrow W$, temos o diagrama comutativo

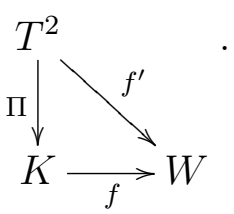

Para encontrar uma aplicação especial $\theta \in[f]$, é necessário encontrar uma aplicação especial $g^{\prime} \in\left[f^{\prime}\right]$. Portanto faremos modificações, por homotopias, na aplicação $f^{\prime}$.

Basta fazer estas modificações na região fundamental $F$ de $T^{2}$ e repetir analogamente as modificações na outra região fundamental.

Como vimos anteriormente, para definir o polinômio associado, existe, a menos de homotopia, um disco $D \in F$ tal que $f^{\prime-1}\left(W \backslash S^{1}\right) \subset D, D$ define o polinômio $p_{[f]}(t)$ e $f^{\prime}(\partial D)=P$. Observando que o polinômio $p_{[f]}(t)$, neste caso, tem coeficientes em $\mathbb{Z}_{2}$, como é explicado no apêndice.

Seja $<x, y ; x y x^{-1} y>$ a apresentação do grupo fundamental de $K$. Suponha que $f_{\pi}(y)=a \neq 0$, como $\pi_{1}(W)$ é abeliano segue que $a^{2}=0$, absurdo. Portanto $f_{\pi}(y)=0$.

Se $f_{\pi}(x)=0$, temos, a menos de homotopia, uma aplicação especial.

Suponha que $f_{\pi}(x)=l \neq 0$. Sem perda de generalidade, podemos considerar que o bordo de $F$ é levado no caminho $x \in K$ pela aplicação $\Pi$. Consideremos a aplicação $h: F \rightarrow S^{1} \subset W$ definida por

$$
h(x)= \begin{cases}f^{\prime}(x) & \text { se } x \in F \backslash \stackrel{\circ}{D} \\ P & \text { se } x \in \stackrel{\circ}{D}\end{cases}
$$

Portanto a aplicação $h$ é uma aplicação de um cilindro em $S^{1}$ tal que $h_{\pi}\left(\pi_{1}(F)\right) \simeq$ $\mathbb{Z}<l>$. É simples verificar que a aplicação $h$, a menos de homotopia, é uma projeção do cilindro em $S^{1}$ como na ilustração abaixo onde cada faixa $\lambda_{i}$ é projetado em $S^{1}$.

Consideremos a aplicação $h^{\prime}: F \rightarrow W$ dada por 


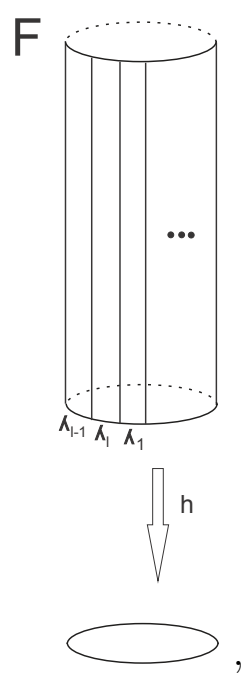

$$
h^{\prime}(x)=\left\{\begin{array}{l}
h(x) \text { se } x \in F \backslash \stackrel{\circ}{D} \\
f^{\prime}(x) \text { se } x \in D
\end{array}\right.
$$

Portanto a partir da aplicação $h^{\prime}$ temos uma aplicação $g \in[f]$ tal que $g$ é especial.

Logo segue o teorema:

Teorema 3.2.2. Seja uma aplicação $f: K \rightarrow W$. Então existe $g \in[f]$ tal que $g$ é uma aplicação especial.

Consideremos o caso em que $g=2 k>2$, então $\mathbb{R} P_{g}^{2} \cong T_{k}^{2} \# K$ e o recobrimento duplo orientável é como na figura 3.2 .

Seja uma aplicação $f: T_{k}^{2} \# K \rightarrow W$. Então, novamente como no caso anterior, para encontrar uma aplicação especial $g \in[f]$ basta fazer modificações na região fundamental de $\widetilde{T_{k}^{2} \# K}$ repetir na outra região do recobrimento e compor com a aplicação $\Pi$. Denotaremos a região fundamental por $F$.

Assim como no caso anterior existe um disco $D \in F$ tal que $f^{\prime-1}\left(W \backslash S^{1}\right) \subset D, D$ define o polinômio $p_{[f]}(t)$ e $f^{\prime}(\partial D)=P$.

Se $f_{\pi}=0$ então, a menos de homotopia, $f$ é especial.

Se $f_{\pi}\left(\pi_{1}\left(\mathbb{R} P_{g}^{2}\right)\right)=<l>$, tal que $l \neq 0$, então da mesma forma como feito no caso de aplicações de $T_{g}$ em $W$, a menos de homeomorfismo, somente um, a menos de homotopia, gerador é levado em $S^{1} \subset W$ e os outros, a menos de homotopia, é levado em $P \in W$. 
Para isso, como $\pi_{1}\left(T_{k}^{2} \# K\right)$ não tem uma base, compomos o homomorfismo induzido por $f$ em grupos fundamentais com a aplicação $\tau: \pi_{1}\left(T_{k}^{2} \# K\right) \rightarrow H_{1}\left(T_{k}^{2} \# K\right)$

Assim como foi feito no caso do toro, podemos considerar $f_{\pi}\left(\alpha_{0}\right)=l$ e $f_{\pi}\left(\alpha_{i}\right)=0$ para $i \neq 0$, onde $\alpha_{i}$ são os geradores de $\pi_{1}\left(T_{k}^{2} \# K\right)$ e $\alpha_{0} \subset T_{k}^{2} \subset T_{k}^{2} \# K$.

Portanto, desde que os bordos de $F$ são levado pela $\Pi$ no gerador $\gamma \pi_{1}\left(T_{k}^{2} \# K\right)$ tal que a imagem de $\gamma$ pela $\Pi$ pertence a $K \in T_{k}^{2} \# K$, a imagem dos bordos de $F$ pela aplicação $f^{\prime}$ é o ponto $P \in W$.

Seja $\partial F=S_{0}^{1} \sqcup S_{1}^{1}$, onde $S_{i}^{1}$ são esferas de dimensão 1 . Tomemos $F$ e identificamos as duas esferas que resulta em $T_{k+1}$ e denotemos $S_{*}^{1}$ as duas esferas identificadas.

Consideremos a aplicação $h: T_{k+1}^{2} \rightarrow W$ dada por

$$
h(x)=\left\{\begin{array}{lll}
f^{\prime}(x) & \text { se } & x \in T_{k+1}^{2} \backslash S_{*}^{1} \\
P & \text { se } & x \in S_{*}^{1}
\end{array}\right.
$$

Pelo Teorema 1.3.1 existe uma aplicação $h^{\prime} \in[h]$ tal que $h^{\prime}$ é aplicação especial e sem perda de generalidade, podemos supor que $h\left(S_{*}^{1}\right)=P$.

Consideremos a aplicação $h^{\prime \prime}: F \rightarrow W$ dada por

$$
h^{\prime \prime}(x)=\left\{\begin{array}{lll}
h^{\prime}(x) & \text { se } & x \in F \backslash S_{0}^{1} \sqcup S_{1}^{1} \\
P & \text { se } & x \in S_{0}^{1} \sqcup S_{1}^{1}
\end{array}\right.
$$

Portanto a partir de $h^{\prime \prime}$ construímos uma aplicação $g: T_{k}^{2} \# K \rightarrow W$ tal que $g \in[f]$ é especial e segue o teorema:

Teorema 3.2.3. Seja uma aplicação $f: T_{k}^{2} \# K \rightarrow W$. Então existe $g \in[f]$ tal que $g$ é uma aplicação especial. 


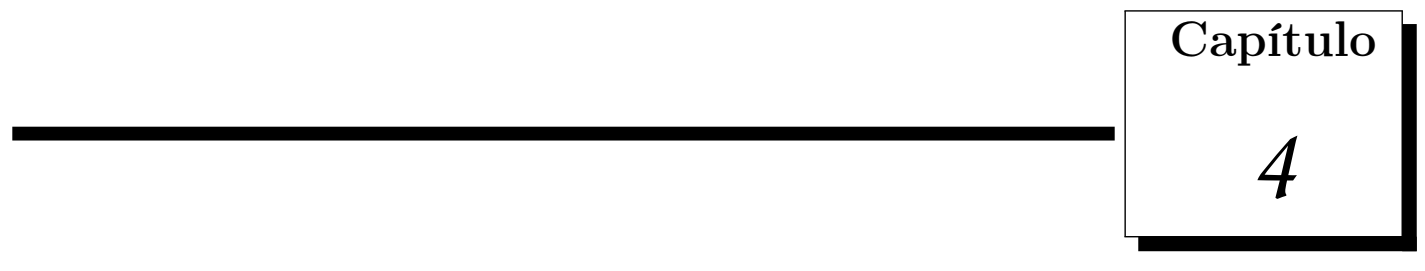

\section{Representação de aplicações de}

\section{superfícies não orientáveis em $W_{n}$}

Nas próximas duas seções, dada uma aplicação $f: \mathbb{R} P_{g}^{2} \rightarrow W_{n}$ tal que $n>1$, é associado um polinômio $p_{[f]}(t)$ a classe de homotopia de $f$, este polinômio tem coeficientes em $\mathbb{Z}_{2}$. Apresenta-se uma aplicação, chamada aplicação especial, a qual no capítulo de ?? mostra-se que estas aplicações realizam o conjunto minimal de raízes, que é construída a partir de um polinômio. Depois, mostra-se que existe uma aplicação especial em cada classe de homotopia $\left[\mathbb{R} P_{g}^{2}, W_{n}\right]$.

O motivo de considerar polinômios com coeficientes em $\mathbb{Z}_{2}$ é explicado no apêndice.

\subsection{Representação das classes de homotopia de aplica- ções de $\mathbb{R} P^{2}$ em $W_{n}$}

Assim como na seção 3.1, consideremos o espaço projetivo $\mathbb{R} P^{2}$ e uma aplicação $f$ : $\mathbb{R} P^{2} \rightarrow W_{n}$, temos o seguinte diagrama comutativo 


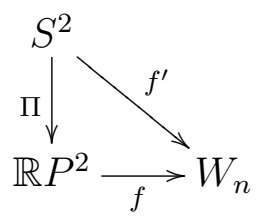

onde $\Pi: S^{2} \rightarrow \mathbb{R} P^{2}$ é o recobrimento duplo orientável.

Como $\pi_{1}\left(\mathbb{R} P^{2}\right) \simeq \mathbb{Z}_{2}$, segue que $f_{\pi}=0$. Logo temos o diagrama

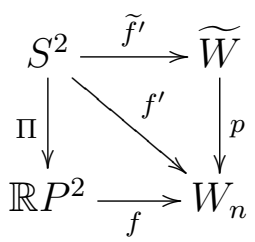

comutativo.

Para fazer modificações, por homotopias, na aplicação $f$ basta fazer modificações numa região fundamental de $S^{2}$ e repetir na outra região e utilizar a aplicação П para modificar $f$. Neste caso como $\Pi$ é recobrimento duplo a região que vamos considerar é $C N \subset S^{2}$ dada como na seção 3.1 .

Sem perda de generalidade podemos supor que $f \circ \Pi\left(S^{1}\right)=P$, onde $S^{1}=\{(x, y, z) \in$ $\mathbb{R}^{3}:|x|^{2}+|y|^{2}+|z|^{2}=1$ e $\left.z=0\right\} \subset S^{2}$.

Identificamos $C N \backslash S^{1} \operatorname{com} S^{2} \backslash N$, para algum $N \in S^{2}$. Consideremos a aplicação $h: S^{2} \rightarrow W_{n}$ definida por

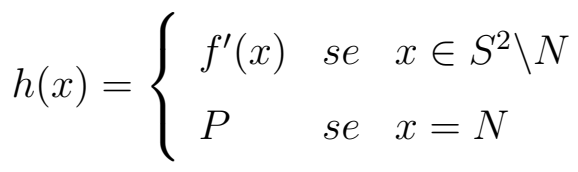

Definimos $\bar{p}_{[h]}(t)=\bar{a}_{0}+\bar{a}_{1} t+\ldots+\bar{a}_{n} t^{n}$ tal que $\bar{a}_{i}$ é igual a $a_{i}$ módulo 2 , onde $p_{[h]}(t)=a_{0}+a_{1} t+\ldots+a_{n} t^{n}$.

Definição 4.1.1. Dada uma aplicação $f: \mathbb{R} P^{2} \rightarrow W_{n}$, definimos o polinômio associado a classe de homotopia $[f]$ por $\bar{p}_{[h]}(t)$, onde $h: S^{2} \rightarrow W_{n}$ é como antes e este polinômio será denotado por $p_{[f]}(t)$.

Dado um polinômio $q(t)=a_{0}+\ldots+a_{n} t^{n}$, tal que os coeficientes de $q(t)$ pertencem a $\mathbb{Z}_{2}$, construiremos uma aplicação $f: \mathbb{R} P^{2} \rightarrow W_{n}$ tal que $p_{[f]}(t)=q(t)$. 
Consideremos a aplicação $h: S^{2} \rightarrow W_{n}$ construída a partir de $q(t)$, como feito na seção 2.1 .

Identificamos a região $C N \backslash S^{1} \operatorname{com} S^{2} \backslash N$, tal que $h(N)=P$.

Dado $y \in \mathbb{R} P^{2}$, então $\Pi^{-1}(y)=\{x,-x\}$ tal que $x \in C N \backslash S^{1}$ e $-x \in \mathbb{R} P^{2} \backslash C N$ ou $\Pi^{-1}(y) \subset S^{1}$.

Definimos $f: \mathbb{R} P^{2} \rightarrow W$ por

$$
f(x)=\left\{\begin{array}{lll}
h(x) & \text { se } & x \in C N \backslash S^{1} \\
P & \text { se } & \Pi^{-1}(y) \subset S^{1}
\end{array}\right.
$$

Por construção $p_{[f]}(t)=q(t)$.

Definição 4.1.2. Uma aplicação $f: \mathbb{R} P^{2} \rightarrow W_{n}$ que pode ser construída como acima é chamada aplicação especial.

Analogamente como feito na seção [3.1, dada uma classe de homotopia $[f]$, pode ser encontrada uma aplicação $g \in[f]$ tal que $g$ é aplicação especial.

\subsection{Representação das classes de homotopia de aplica- ções de $\mathbb{R} P_{g}^{2}$ em $W_{n}$}

Como na seção 3.2 consideremos as superfícies não orientáveis $\mathbb{R} P_{g}^{2}$ tal que $g=2 k+1 \geq$ 2 e, portanto, $\mathbb{R} P_{g}^{2} \cong T_{k}^{2} \# \mathbb{R} P^{2}$.

Seja $\widetilde{\mathbb{R} P_{g}^{2}}$ o recobrimento duplo orientável de $\mathbb{R} P_{g}^{2}$ como na figura 3.2 ,

Portanto temos um diagrama comutativo como abaixo

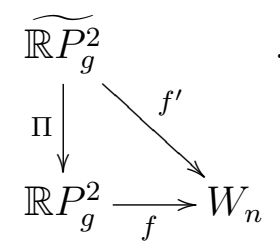

Da mesma forma que na seção 3.2 temos a definição:

Definição 4.2.1. Dadas uma aplicação $f: \mathbb{R} P_{g}^{2} \rightarrow W_{n}$ e $f^{\prime}: \widetilde{\mathbb{R} P_{g}^{2}} \rightarrow W_{n}$ tal que 
$f \circ \Pi=f^{\prime}$, como no diagrama acima. Então o polinômio associado a aplicação $f$ é $\bar{p}_{\left[f^{\prime}\right]}(t)$ e será denotado por $p_{[f]}(t)$.

\section{Aplicação especial de $\mathbb{R} P_{g}^{2}$ em $W_{n}$ para $g=2 k+1$}

Dados um polinômio $q(t)=a_{0}+\ldots+a_{n} t^{n}$ tal que $a_{0} \neq 0, a_{i} \in \mathbb{Z}_{2}$ e um número $l \in \mathbb{N}$ finito.

Construímos uma aplicação $f: T_{k}^{2} \rightarrow W_{n}$ a partir de $q(t)$ e $l$, como feito na seção 2.2 .

Sem perda de generalidade podemos supor que existe um disco fechado $D \subset T_{g}^{2}$ tal que $f(D)=P$. Identificamos o subconjunto $C=T_{k}^{2} \backslash \stackrel{\circ}{D}$ com uma região básica do recobrimento duplo orientável de $\mathbb{R} P_{g}^{2}$.

Definimos a aplicação $h: \mathbb{R} P_{g}^{2} \rightarrow W_{n}$ por $h([x])=f(x)$, onde $\Pi^{-1}([x]) \cap C=x$.

Por construção temos $h_{\pi}\left(\pi_{1}\left(\mathbb{R} P_{g}^{2}\right)\right) \simeq \mathbb{Z}<l>$ e $p_{[h]}(t)=q(t)$.

Definição 4.2.2. Uma aplicação $h: \mathbb{R} P_{g}^{2} \rightarrow W_{n}$ é chamada aplicação especial se $h$ pode ser construída a partir de l finito e seu polinômio associado $p_{[h]}(t)$ como a construção acima.

Pelos mesmos argumentos feitos no caso de aplicação de $\mathbb{R} P_{g}^{2}$ em $W$, dada uma aplicação $f: \mathbb{R} P_{g}^{2} \rightarrow W_{n}$ existe uma aplicação $\theta \in[f]$ tal que $\theta$ é especial.

\section{Aplicação especial de $\mathbb{R} P_{g}^{2}$ em $W_{n}$ para $g=2 k$}

Dados um polinômio $q(t)=a_{0}+\ldots+a_{n} t^{n}$ tal que $a_{0} \neq 0, a_{i} \in \mathbb{Z}_{2}$ e um número $l \in \mathbb{N}$ finito.

Construímos uma aplicação $f: T_{k+1}^{2} \rightarrow W_{n}$ a partir de $q(t)$ e $l$, como feito na seção 2.2 .

Seja uma esfera $S_{*}^{1} \in T_{k+1}^{2}$ tal que $S_{*}^{1}$ não é contrátil em $T_{k+1}^{2}$ e $f\left(S_{*}^{1}\right)=x_{0}$. Identificamos o subconjunto $T_{k+1}^{2} \backslash S_{*}^{1} \operatorname{com} F \backslash \partial F$, onde $F$ é uma região básica do recobrimento duplo orientável de $\mathbb{R} P_{g}^{2}$.

Definimos a aplicação $h: \mathbb{R} P_{g}^{2} \rightarrow W_{n}$ por $h([x])=f(x)$, onde $\Pi^{-1}([x]) \cap F=x$. 
Por construção temos $h_{\pi}\left(\pi_{1}\left(\mathbb{R} P_{g}^{2}\right)\right) \simeq \mathbb{Z}<l>, p_{[h]}(t)=q(t)$.

Definição 4.2.3. Uma aplicação $h: \mathbb{R} P_{g}^{2} \rightarrow W_{n}$ é chamada aplicação especial se $h$ pode ser construída a partir de l finito e seu polinômio associado $p_{[h]}(t)$ como a construção acima.

Pelos mesmos argumentos feitos no caso de aplicação de $\mathbb{R} P_{g}^{2}$ em $W$ junto com as técnicas usadas na seção 2.3 para provar a existência de aplicação especial, então dada uma aplicação $f: \mathbb{R} P_{g}^{2} \rightarrow W_{n}$ existe uma aplicação $g \in[f]$ tal que $g$ é especial.

Teorema 4.2.1. Seja uma aplicação $f: \mathbb{R} P_{g}^{2} \rightarrow W_{n}$. Então existe uma aplicação $g \in[f]$ tal que g é aplicação especial. 


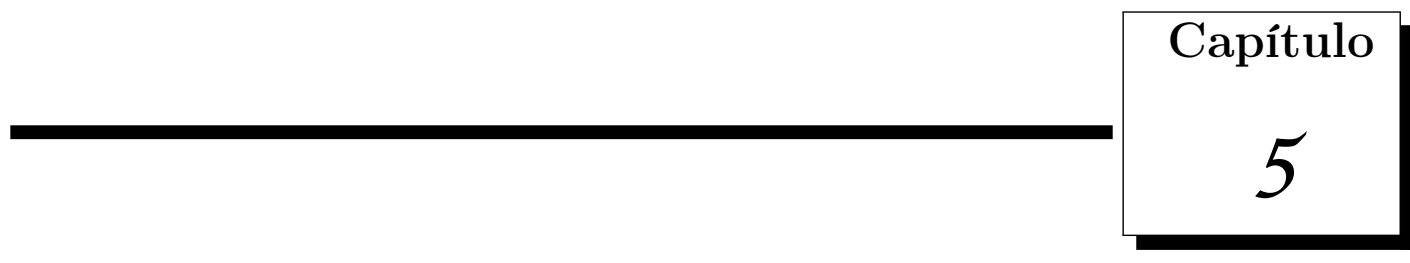

Raízes de aplicações de superfícies orientáveis em $W_{n}$

Seja $f: S \rightarrow W_{n}$ uma aplicação contínua. Neste capítulo é feito um estudo dos conjuntos $f^{-1}(x), \forall x \in W_{n}$, tal que $g^{-1}(x)$ seja o conjunto minimal e $g \in[f]$, onde a minimalidade se refere a $g^{-1}(x)$ ser o conjunto com menor dimensão, menor número de componentes conexas e as dimensões dos grupos de homologias de $g^{-1}(x)$ são minimais.

Para cada $x \in W_{n}$, denotaremos o conjunto minimal de raízes por $M(f, x)=\left\{g^{-1}(x)\right.$ é minimal : $g \in[f]\}$.

Observe que para quaisquer $x, y \in W_{n} \backslash \bigsqcup_{i=1}^{n} S_{i}^{2}$, temos $M(f, x)=M(f, y)$ e para quaisquer $x, y \in \bigsqcup_{i=1}^{n}\left(S_{i}^{2} \backslash\{i\}\right) \subset W_{n}$ também vale $M(f, x)=M(f, y)$.

\subsection{Raízes de aplicações de $S^{2}$ em $W_{n}$}

Consideremos $n=1$ e uma aplicação $f: S^{2} \rightarrow W$. Quando $f\left(S^{2}\right) \subset S^{1}$ ou quando $p_{[f]}(t)=\mathbb{O}$, então $f$ é homotópica a uma aplicação constante. $\operatorname{Logo} M(f, x)=\emptyset$, para qualquer $x \in W$.

Se $p_{[f]}(t)=a_{0} \neq 0$, então neste caso o diagrama 


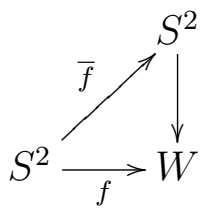

é comutativo e $a_{0}$ é o grau de $\bar{f}$, o conjunto minimal de raízes para $x \in S^{2} \subset W$ é unitário e para $x \in W \backslash S^{2} M(f, x)=\emptyset$.

De fato, suponha que existe $x \in W \backslash S^{1}$ tal que $f^{-1}(x)=\emptyset$. Logo fazendo uma retração temos $f\left(S^{2}\right) \subset S^{1}$, o que implicaria $f \cong c t e$, contradizendo $p_{[f]}(t)=a_{0} \neq 0$.

Se $x \in W \backslash S^{2}$, então pelo diagrama comutativo acima, temos $M(f, x)=\emptyset$.

Portanto se $p_{[f]}(t)=a_{0} \neq 0$, temos os seguintes conjuntos minimais de raízes

$$
M(f, x)= \begin{cases}\emptyset & , \quad \text { se } x \in W \backslash S^{2} \\ \{y\} & , \quad \text { se } x \in S^{2} \subset W\end{cases}
$$

Suponha que $p_{[f]}(t)=a_{0}+\ldots+a_{n} t^{n} \operatorname{com} a_{0} \neq 0, a_{n} \neq 0$ e $n>0$.

Logo a aplicação $f$ é fortemente sobrejetiva e para cada $x \in W$ temos a imagem inversa $f^{-1}(x) \neq \emptyset$. Para $x \in W \backslash S^{1}$ uma aplicação especial tem, a menos de homotopia, como imagem inversa um único ponto, isto é, $f^{-1}(x)=\{y\}$, logo uma aplicação especial realiza o conjunto minimal de raízes, neste caso. Provemos que para $x \in W \backslash S^{2}$ uma aplicação especial realiza o conjunto minimal de raízes.

Para $x \in W \backslash S^{2}$ temos as seguintes possibilidades:

(i) $M(f, x) \cong S_{0}$;

(ii) $M(f, x) \cong S_{1}$;

(iii) $M(f, x) \cong S_{2}$;

onde $S_{i}$ são subespaços topológicos de $W$ de dimensões $i=0,1$ ou 2 , podendo não ser conexo.

Se acontecesse $(i)$, então para cada $y \in f^{-1}(x)$ é tal que $y$ é raiz isolada de $f$ em $x$.

Sejam $V$ uma vizinhança de $y$ tal que $y$ seja a única raiz de $f$ em $x$ pertencente a $V$ e $\{\widetilde{x}\}=\widetilde{f}(V) \cap p^{-1}(x) \subset \widetilde{W}$. Então por continuidade, $\widetilde{f}(V \backslash\{x\}) \subset[\widetilde{x},+\infty)$ ou $\widetilde{f}(V \backslash\{x\}) \subset(-\infty, \widetilde{x}]$. Logo, a menos de homotopia, $\widetilde{f}(V) \cap\{\widetilde{x}\}=\emptyset$ e, portanto, amenos de homotopia, $f(V) \cap\{x\}=\emptyset$. Contradiz o fato de $f$ ser fortemente sobrejetiva. 
Se vale (iii), então cada componente conexa de $S_{2}$ é homotópica a $D^{2}$ ou é toda $S^{2}$. Caso a componente conexa seja homotópica a $D^{2}$, então deste que $D^{2} \cong p t o$, a menos de homotopia, a componente conexa é um único ponto e novamente usando os argumentos do caso (ii) temos que este caso não acontece.

Se $S_{2}$ é todo o domínio de $f$, então $f$ uma aplicação constante o que contradiz $p_{[f]}(t) \neq$ (1).

Portanto, vale (ii).

Cada componente conexa de $S_{1}$ é homotópica a $[0,1]$ ou é homotópica a $S^{1}$.

Suponha que a componente conexa de $S_{1}$ é homotópica a $[0,1]$. Como $[0,1] \cong p t o$, a menos de homotopia, es ta componente conexa é um único ponto. Logo repetimos os argumentos de $(i i)$ e chegamos numa contradição. Portanto, temos somente o caso $S_{1} \cong \bigsqcup_{i=1}^{r} S_{i}^{1}$

Observe que $p^{-1}(x) \cap \widetilde{f}\left(S^{2}\right)$ é pelo menos $n$ pontos e para cada um destes ponto a imagem inversa é por $\widetilde{f}$ é homotópica a uma união finita de $S^{1}$.

Para $f$ uma aplicação especial $p^{-1}(x) \cap \widetilde{f}\left(S^{2}\right)$ é exatamente $n$ pontos e, a menos de homotopia, a imagem inversa de cada um deste pontos é uma única $S^{1}$. Portanto uma aplicação especial realiza o conjunto minimal de raízes parar $x \in W \backslash S^{2}$.

Finalmente o ponto $P \in W$ singular. Como já vimos $f^{-1}(P) \neq \emptyset$.

Se $x \in f^{-1}(P)$ tal que $x$ é raiz isolada, então assim como nos casos anteriores esta raiz pode ser eliminada por homotopias, lembrando que estamos no caso de $f$ fortemente sobrejetiva.

Da mesma forma como feito anteriormente, não acontecem os casos $M(f, P)$ ser homotópico subespaços de dimensão 2

Logo $M(f, P)$ contém somente espaços de dimensão 1 .

Consideremos $\tilde{f}: S^{2} \rightarrow \widetilde{W}$ um levantamento de aplicação especial $f$ ao recobrimento universal e seja o conjunto $\{0, \ldots, n\}=\widetilde{f}\left(S^{2}\right) \cap \mathbb{Z}$. Observe que $n$ é mínimo.

$\operatorname{Logo} f^{-1}(P)=\widetilde{f}^{-1}(\{0, \ldots, n\})$.

Os espaços de dimensão 1 que estão contidos em $f^{-1}(P)$ tem mesmo tipo de homotopia que buquês de esferas de dimensão 1 , isto é, $\underbrace{S^{1} \vee \ldots \vee S^{1}}_{j-\text { vezes }}$, para algum $j \in \mathbb{N}$. 
Para cada $i \in\{0, \ldots, n\}$ temos $\tilde{f}^{-1}(i)$ contendo pelo menos um espaço do mesmo tipo de homotopia que $S^{1}$.

Portanto no caso de $i=0, n$ ou $i \in\{1, \ldots, n-1\}$ tal que $a_{i}=0$ temos $\widetilde{g}^{-1}(i)=S^{1}$ que é o conjunto minimal de raízes.

Para $i \in\{1, \ldots, n-1\}$ tal que $a_{i} \neq 0$, uma aplicação especial $f$ tem o conjunto $f^{-1}(i)=S^{1} \vee S^{1}$ como imagem inversa. Suponha que o conjunto minimal de raízes de $i$ seja $S^{1}$, isto é, existe $h \in[f]$ tal que $h^{-1}(i)=S^{1}$. Seja $V$ uma vizinhança tubular de $S^{1}$ em $S^{2}$. Como $a_{i} \neq 0$ temos $h(V) \cap\left(S_{i}^{2} \backslash\{i\}\right) \neq \emptyset, h(V) \cap(i,+\infty) \neq \emptyset$ e $h(V) \cap(-\infty, i) \neq \emptyset$. Para $V$ suficientemente pequeno $V \backslash S^{1}$ tem somente duas componentes conexas. Logo por conexidade não existe $h$.

Portanto, a menos de homotopia, $f^{-1}(P)=\underbrace{S^{1} \vee \ldots \vee S^{1}}_{n-\text { vezes }} \operatorname{com} n \geq 2$.

Logo uma aplicação especial realiza o conjunto minimal de raízes para $P \in W$.

Concluímos que para qualquer $x \in W$ existe uma aplicação especial $f: S^{2} \rightarrow W$ que realiza $M(f, x)$ e este conjunto é classificado pelo polinômio $p_{[f]}(t)=a_{0}+\ldots+a_{n} t^{n}$ tal que $a_{0} \neq=0$ e $a_{n} \neq 0$. Definimos $r=\#\left\{a_{i} ; a_{i} \neq 0\right\}$ e observe que $r \neq 2$. Então o minimal de raízes é como abaixo,

$$
M(f, x)= \begin{cases}\text { tem cardinalidade } r & , \text { se } x \in W \backslash S^{1} \\ \bigsqcup_{j=1}^{n} S_{j}^{1} & , \text { se } x \in W \backslash S^{2} \\ \left(\bigsqcup_{j=1}^{r-2}\left(S^{1} \vee S^{1}\right)_{j}\right) \cup\left(\bigsqcup_{j=1}^{n-r+3} S_{j}^{1}\right), & \text { se } x=P\end{cases}
$$

Abordaremos minimalidade de raízes no caso de aplicações $f: S^{2} \rightarrow W_{n}$ tal que $n \geq 2$. Consideremos $n \geq 2$ e uma aplicações $f: S^{2} \rightarrow W_{n}$. Suponha que $f$ é uma aplicação especial.

Fixemos o recobrimento $p^{\prime}: \widetilde{W} \rightarrow W_{n}$, como na seção 2.1. Sem perda de generalidade podemos supor que $p^{\prime}\left(S_{0}^{2}\right)=e_{0}^{2}$.

Da mesma maneira como feito no caso de aplicações de $S^{2}$ em $W$, mostrar-se que as 
aplicações especiais são as aplicações que realizam o conjunto minimal de raízes para os pontos de $W_{n}$.

Seja $p_{[f]}(t)=a_{0}+\ldots+a_{k} t^{k}$ o polinômio associado a $f$. Se $p_{[f]}(t)$ é o polinômio nulo, então $M(f, x)=\emptyset$ para qualquer $x \in W_{n}$. Suponha que $p_{[f]}(t)=a_{0} \neq 0$, então o conjunto minimal de raízes é

$$
M(f, x)=\left\{\begin{array}{lll}
\{y\} & \text { se } & x \in e_{0}^{2} \\
\emptyset & \text { c.c }
\end{array}\right.
$$

Suponha que $k>1$ com $a_{0} \neq 0$ e $a_{k} \neq 0$. Para cada $h, h^{\prime} \in\{0, \ldots, n\}$, consideremos $c_{h}$ a cardinalidade do conjunto $\left\{h+j n / h+j n \in\{0, \ldots, k\}, a_{h+j n} \neq 0\right.$ e $\left.j \in \mathbb{N}\right\}$ e $c_{h^{\prime}}$ é a cardinalidade do conjunto $\left\{h^{\prime}+j n / h^{\prime}+j n \in\{0, \ldots, k\}, a_{h^{\prime}+j n}=0\right.$ e $\left.j \in \mathbb{N}\right\}$. Suponha que $k=m n+r$ para certos $m, r \in \mathbb{N}$.

Portanto o conjunto minimal de raízes neste caso é

$$
M(f, x)= \begin{cases}\bigsqcup_{j=1}^{\text {tem cardinalidade } c_{h}} \quad, \quad \text { se } x \in e_{i}^{2} \backslash\left\{x_{i}\right\} \\ \bigsqcup_{j=1}^{m+1} S_{j}^{1} & , \quad \text { se } x \in\left(e_{i}^{1} \backslash\left\{x_{i}, x_{i+1}\right\}\right) \text { e } 1 \leq i \leq r \\ \left(\bigsqcup_{j=1}^{c_{h}}\left(S^{1} \vee S^{1}\right)_{j}\right) \cup\left(\bigsqcup_{j=1}^{c_{h^{\prime}}} S_{j}^{1}\right) & , \quad \text { se } x \in\left(e_{i}^{1} \backslash\left\{x_{i}, x_{i+1}\right\}\right) \text { e } r<i \leq n \\ \left(\bigsqcup_{j=1}^{c_{h}-2}\left(S^{1} \vee S^{1}\right)_{j}\right) \cup\left(\bigsqcup_{j=1}^{c_{h^{\prime}+2}+2} S_{j}^{1}\right), & \text { se } x=x_{i} \text { e } i \neq 0, r\end{cases}
$$

\subsection{Raízes de aplicações de $T^{2}$ em $W_{n}$}

Sejam $f: T^{2} \rightarrow W$ uma aplicação especial, $p_{[f]}(t)$ o polinômio associado a classe de homotopia $[f], n=1$ e $l \in N$ tal que $\left\langle l>=f_{\pi}\left(\pi_{1}\left(T^{2}\right)\right)\right.$.

Provemos que uma aplicação especial é a aplicação que realiza o conjunto minimal de raízes para qualquer $x \in W$.

Se $p_{[f]}(t)=\mathbb{O}$ e $l=0$ então $f$ é homotópica a uma aplicação constante. Portanto $M(f, x)=\emptyset$ para qualquer $x \in W$. 
Se $p_{[f]}(t) \neq \mathbb{O}$, então pelo resultados da seção 1.2 existe $D \subset T^{2}$ tal que $\left.f\right|_{(D, \partial D)}$ é dada a partir do polinômio $p_{[f]}(t)$ como aplicação de $S^{2}$ em $W$.

Como $f^{-1}\left(W \backslash S^{1}\right) \subset D$, então para todo $x \in W \backslash S^{1}$ o conjunto minimal de raízes é obtido como feito no caso de minimalidade de raízes de aplicação de $S^{2}$ em $W$.

Sejam $x \in W \backslash S^{2}$ e $l \geq 1$, então o conjunto minimal de raízes contém ao menos $f^{-1}(x) \cap$ $D$. A aplicação especial tem como conjunto de raízes $\left(f^{-1}(x) \cap D\right) \sqcup\left(l\right.$ copias de $\left.S^{1}\right)$.

Consideremos a aplicação $\bar{f}: C \rightarrow \widetilde{W}$ e $p: \widetilde{W} \rightarrow W$ como na seção ??, então $f^{-1}(x)=\bar{f}^{-1}\left(\left\{\bar{x}_{0}, \ldots, \bar{x}_{l}\right\}\right)$, onde $\bar{x}_{i} \in(i, i+1) \subset \widetilde{W}, i \in\{0, \ldots, l\}$ e $p\left(\bar{x}_{i}\right)=x$.

Suponha que para algum $i \in\{0, \ldots, l\}$ o conjunto $\bar{f}^{-1}\left(\bar{x}_{i}\right) \cap(C \backslash D)=S^{1}$ não seja o conjunto minimal, isto é, existe $g \in[f]$ tal que $\bar{g}^{-1}\left(\bar{x}_{i}\right) \subset S^{1} \backslash\{y\}$, para algum ponto $y$.

Como o conjunto $S^{1} \backslash\{y\}$ tem mesmo tipo de homotopia que um ponto, então, a menos de homotopia, $\bar{g}\left(\bar{x}_{i}\right) \cap(C \backslash D)$ é um único ponto. Portanto, por homotopia, podemos eliminar esta raiz e $\bar{g}\left(\bar{x}_{i}\right) \cap(C \backslash D)=\emptyset$.

Logo, a menos de homotopia, o conjunto minimal $\bar{f}^{-1}\left(\bar{x}_{i}\right)$ é $S^{1}$.

Analogamente pode ser feito no caso do ponto $P \in W$ para concluir que o conjunto $f^{-1}(P)=\bar{f}^{-1}(\{0, \ldots, l\})=\underbrace{S^{1} \sqcup \ldots \sqcup S^{1}}_{l-\text { vezes }} \sqcup\left(S^{1} \vee S^{1}\right)$, onde $\bar{f}^{-1}(i)=S^{1}$ para $i \in\{1, \ldots, l\}$ e $\bar{f}^{-1}(0)=S^{1} \vee S^{1}$

Concluindo assim que o conjunto minimal de raízes de uma aplicação de $T^{2}$ em $W$ é o conjunto de raízes de uma aplicação especial tal que o conjunto de raízes é classificado pelo polinômio $p_{[f]}(t)=a_{0}+\ldots+a_{n} t^{n}$ e $l \in \mathbb{N}$ tal que $<l>=f_{\pi}\left(\pi_{1}\left(T^{2}\right)\right)$.

Portanto, se $a_{i}=0$ para todo $i \in\{0, \ldots, n\}$ e $l=0$, então $M(f, x)=\emptyset$ para todo $x \in W$.

Se $p_{[f]}(t)=a_{0} \neq 0$ e $l=0$, o conjunto minimal de raízes é $M(f, x)=\emptyset$ para $x \in W \backslash S^{2}$ e $M(f, x)$ é unitário caso contrário.

Se $a_{0} \neq 0, a_{n} \neq 0, n \geq 1$ e $l=0$, então o conjunto minimal de raízes é 


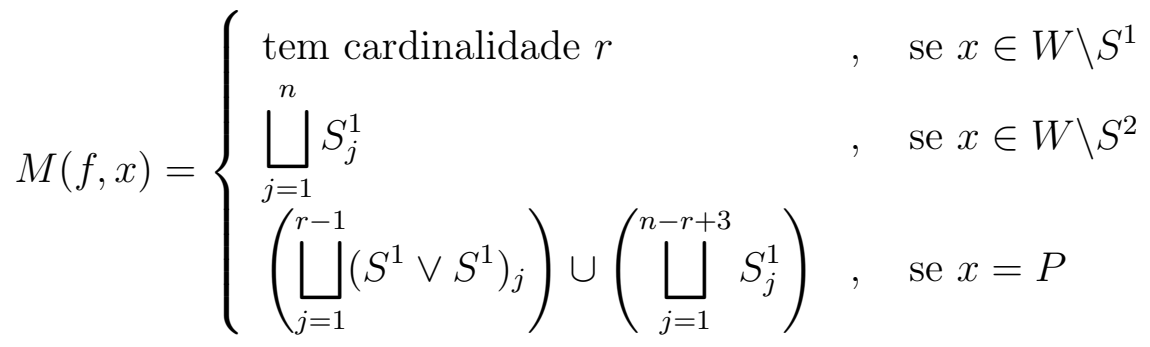

onde $r=\#\left\{a_{i} ; a_{i} \neq 0\right\}$.

Caso contrário, isto é, $a_{0} \neq 0, a_{n} \neq 0, n \geq 1, l \neq 0$. Seja que $r=\#\left\{a_{i} ; a_{i} \neq 0\right\}$, então o conjunto minimal de raízes é como abaixo,

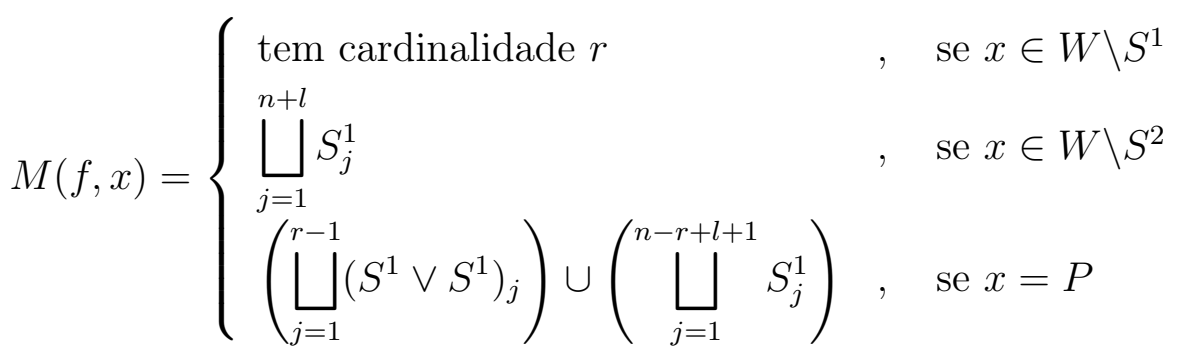

Consideremos $n \geq 2$ e uma aplicações $f: T^{2} \rightarrow W_{n}$ especial.

Fixemos o recobrimento $p^{\prime}: \widetilde{W} \rightarrow W_{n}$ tal que $p^{\prime}\left(S_{0}^{2}\right)=e_{0}^{2}$.

Da mesma maneira com feito anteriormente nesta seção, mostra-se que as aplicações especiais são as aplicações que realizam o conjunto minimal de raízes para os pontos de $W_{n} \operatorname{com} n \geq 2$.

Sejam $p_{[f]}(t)=a_{0}+\ldots+a_{k} t^{k}$ o polinômio associado a $f$ e $l \in \mathbb{N}$ tal que $f_{\pi}\left(\pi_{1}\left(T^{2}\right)\right)=$ $\mathbb{Z}<l>$. Se $p_{[f]}(t)$ é o polinômio nulo e $l=0$, então o conjunto minimal de raízes é vazio para qualquer ponto de $W_{n}$.

Suponha que $p_{[f]}(t)=a_{0} \neq 0$ e $l=0$, então o conjunto minimal de raízes é

$$
M(f, x)=\left\{\begin{array}{lll}
\{y\} & \text { se } & x \in e_{0}^{2} \\
\emptyset & & \text { c.c }
\end{array}\right.
$$

Suponha que $a_{0} \neq 0, a_{k} \neq 0, k>0, l=0, p^{\prime}\left(S_{0}^{2}\right)=e_{0}^{2}$ e $p^{\prime}\left(S_{k}^{2}\right)=e_{r}^{2}$ para algum $r \in\{1, \ldots, n\}$. Para cada $h, h^{\prime} \in\{0, \ldots, n\}$, consideremos $c_{h}$ a cardinalidade do conjunto 
$\left\{h+j n / h+j n \in\{0, \ldots, k\}, a_{h+j n} \neq 0\right.$ e $\left.j \in \mathbb{N}\right\}$ e $c_{h^{\prime}}$ é a cardinalidade do conjunto $\left\{h^{\prime}+j n / h^{\prime}+j n \in\{0, \ldots, k\}, a_{h+j n}=0\right.$ e $\left.j \in \mathbb{N}\right\}$. Suponha que $k=m n+r$ para alguns $m, r \in \mathbb{N}$.

Portanto o conjunto minimal de raízes neste caso é

$$
M(f, x)= \begin{cases}\bigsqcup_{j=1}^{\text {tem cardinalidade } c_{h}} S_{j}^{1} \quad, \quad \text { se } x \in e_{i}^{2} \backslash\left\{x_{i}\right\} \\ \bigsqcup_{j=1}^{m+1} S_{j}^{1} & , \quad \text { se } x \in\left(e_{i}^{1} \backslash\left\{x_{i}, x_{i+1}\right\}\right) \text { e } 1 \leq i \leq r \\ \left(\bigsqcup_{j=1}^{c_{h}}\left(S^{1} \vee S^{1}\right)_{j}\right) \cup\left(\bigsqcup_{j=1}^{c_{h^{\prime}}} S_{j}^{1}\right) & , \quad \text { se } x \in\left(e_{i}^{1} \backslash\left\{x_{i}, x_{i+1}\right\}\right) \text { e } r<i \leq n \\ \left(\bigsqcup_{j=1}^{c_{h}-2}\left(S^{1} \vee S^{1}\right)_{j}\right) \cup\left(\bigsqcup_{j=1}^{c_{h^{\prime}}+2} S_{j}^{1}\right), & \text { e } i \neq 0, r \\ \left(\bigsqcup_{j=1}^{c_{h}-1}\left(S^{1} \vee S^{1}\right)_{j}\right) \cup\left(\bigsqcup_{j=1}^{c_{h^{\prime}}+1} S_{j}^{1}\right), & \text { se } x=x_{0}\end{cases}
$$

Por fim, suponha que $a_{0} \neq 0, a_{k} \neq 0, k>0, l \neq 0, p^{\prime}\left(S_{0}^{2}\right)=e_{0}^{2}$ e $p^{\prime}\left(S_{k}^{2}\right)=e_{r}^{2}$ para algum $r \in\{1, \ldots, n\}$. Para cada $h, h^{\prime} \in\{0, \ldots, n\}$, consideremos $c_{h}$ a cardinalidade do conjunto $\left\{h+j n / h+j n \in\{0, \ldots, k\}, a_{h+j n} \neq 0\right.$ e $\left.j \in \mathbb{N}\right\}$ e $c_{h^{\prime}}$ é a cardinalidade do conjunto $\left\{h^{\prime}+j n / h^{\prime}+j n \in\{0, \ldots, k\}, a_{h^{\prime}+j n}=0\right.$ e $\left.j \in \mathbb{N}\right\}$. Suponha que $k=m n+r$ para alguns $m, r \in \mathbb{N}$.

Logo o conjunto minimal de raízes é 


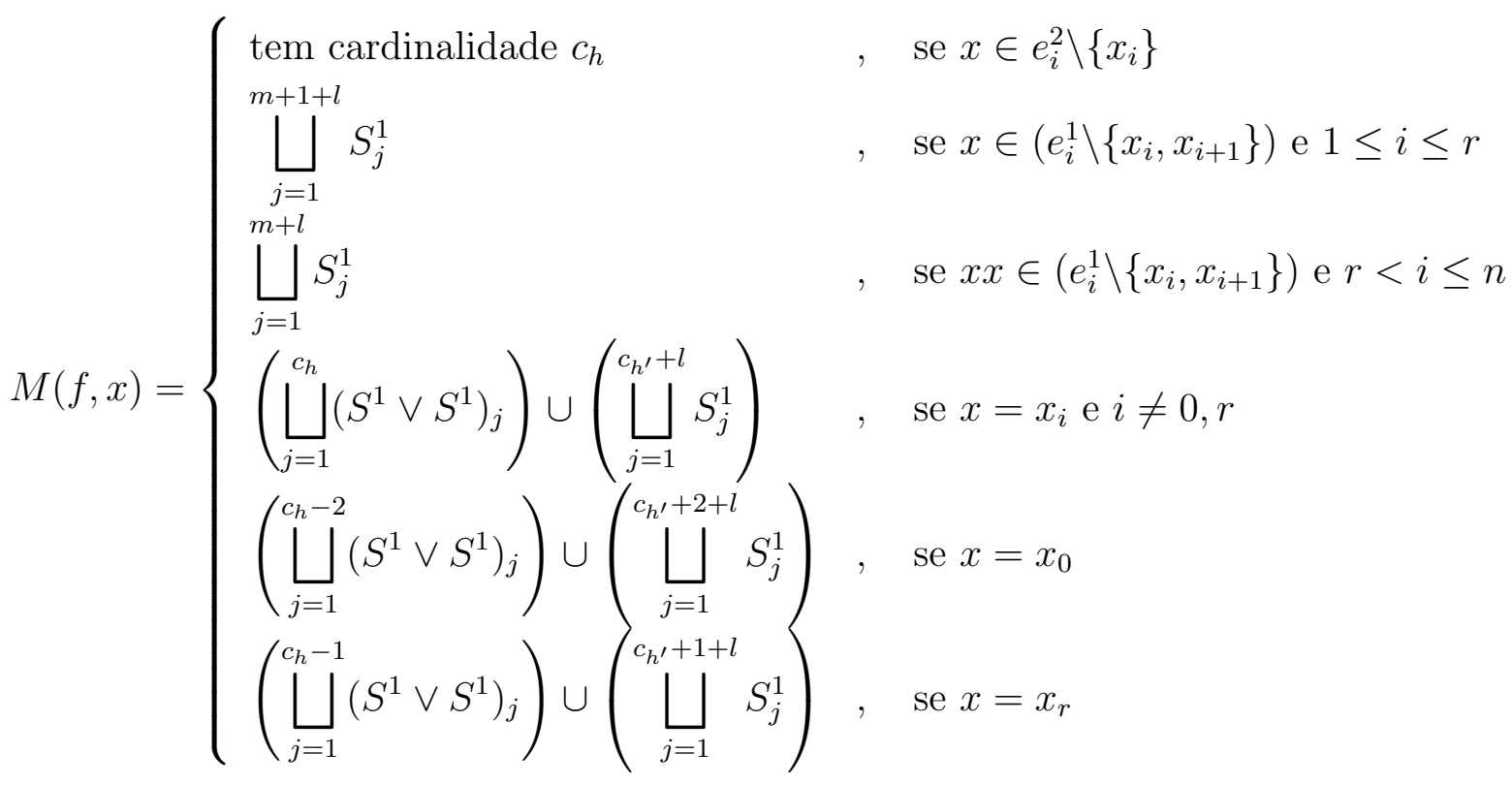

\subsection{Raízes de aplicações de $T_{g}$ em $W_{n}$}

Assim como nos casos anteriores provaremos que o conjunto de raízes de uma aplicação especial $f: T_{g} \rightarrow W$ é minimal.

Sejam $n=1$, uma aplicação especial $f: T_{g} \rightarrow W, p_{[f]}(t)$ o polinômio associado a classe de homotopia $[f]$ e $l \in N$ tal que $<l>=f_{\pi}\left(\pi_{1}\left(T_{g}\right)\right)$.

Se $p_{[f]}(t)=\mathbb{O}$ e $l=0$ então $f$ é homotópica a uma aplicação constante. Portanto o conjunto minimal de raízes é vazio para qualquer $x \in W$.

Suponha que $p_{[f]}(t) \neq \mathbb{O}$ e $l \neq 0$. Então sabemos que existe $D \subset T_{g}$ tal que $\left.f\right|_{(D, \partial D)}$ é dada a partir do polinômio $p_{[f]}(t)$ como aplicação de $S^{2}$ em $W$.

Portanto $\left.f\right|_{(D, \partial D)}$ tem conjunto minimal de raízes. Como $f^{-1}\left(W \backslash S^{1}\right) \subset D$, então para todo $x \in W \backslash S^{1}$ o conjunto minimal de raízes é obtido como feito no caso de minimalidade de raízes de aplicação de $S^{2}$ em $W$.

Seja $x \in W \backslash S^{2}$. Então o conjunto minimal de raízes é ao menos o conjunto $f^{-1}(x) \cap D$. A menos de homotopia, uma aplicação especial tem como conjunto de raízes o seguinte:

$$
\left\{\left(f^{-1}(x) \cap D\right) \bigsqcup\left(l \text { copias de } S^{1}\right)\right\}
$$


Consideremos a aplicação $\left.\bar{f}\right|_{B}: B \rightarrow \widetilde{W}$, então $f^{-1}(x)=\bar{f}^{-1}\left(\left\{\bar{x}_{0}, \ldots, \bar{x}_{l}\right\}\right)$, onde $\bar{x}_{i} \in(i, i+1) \subset \widetilde{W}, i \in\{0, \ldots, l\}$ e $p^{\prime}\left(\bar{x}_{i}\right)=x$.

Analogamente ao caso de raízes de aplicações de $T^{2}$ em $W$ se prova que este é o conjunto minimal de raízes e para o ponto $P \in W$ o conjunto minimal de raízes é $f^{-1}(P)=$ $f^{-1}(\{0, \ldots, l\})=\underbrace{S^{1} \sqcup \ldots \sqcup S^{1}}_{l \text {-vezes }} \sqcup\left(S^{1} \vee S^{1}\right)$, onde $\bar{f}^{-1}(i)=S^{1}$ para $i \in\{1, \ldots, l\}$ e $\bar{f}^{-1}(0)=$ $S^{1} \vee S^{1}$

Concluindo assim que o conjunto minimal de raízes de uma aplicação de $T_{g}$ em $W$ é o mesmo conjunto de raízes de uma aplicação especial de $T^{2}$ em $W$.

Analogamente como feito no caso de $T_{g}$ em $W$, concluímos que no caso de $n>2$, o conjunto minimal de raízes é igual ao caso de aplicações de $T^{2}$ em $W_{n}$. 


\title{
Capítulo
}

6

\section{Raízes de aplicações de superfícies não}

\author{
orientáveis em $W_{n}$
}

\subsection{Raízes de aplicações de $\mathbb{R} P^{2}$ em $W_{n}$}

Dados uma aplicação $f: \mathbb{R} P^{2} \rightarrow W$ e $\Pi: S^{2} \rightarrow \mathbb{R} P^{2}$ o recobrimento duplo orientável.

Na seção 3.1 foi considerado $C N \subset S^{2}$ para fazer modificações, por homotopias, e concluir os resultados. Nesta seção, também, basta utilizar a aplicação $f \circ \Pi$ e $C N \subset S^{2}$ para obter os conjuntos minimais de raízes.

Uma aplicação especial $f: \mathbb{R} P^{2} \rightarrow W$ é construída a partir de uma aplicação especial $h: S^{2} \rightarrow W$. Portanto como uma aplicação especial $h$ realiza o conjunto minimal de raízes para pontos de $W$, então uma aplicação especial $f$ também será uma aplicação que realiza o conjunto minimal de raízes.

Desde que uma aplicação especial $f$ é construída identificando $S^{2} \backslash\{N\} \operatorname{com} C N \backslash S^{1}$, onde $h(N)=P$, o conjunto minimal de raízes de $f$ é igual ao conjunto de raízes de $h$ para $x \in W \backslash\{P\}$ e no ponto $P$ o conjunto minimal de raízes é igual ao conjunto minimal de raízes de $h$ em $P$ mais uma $S^{1}=\Pi(\partial C N)$.

Portanto seja $p_{[f]}(t)$ o polinômio associado a $f$. Se $p_{[f]}(t)=\mathbb{O}$, então, a menos de homotopia, $f^{-1}(x)=\emptyset$ para cada $x \in W$. 
Caso $p_{[f]}(t)=1$, então, a menos de homotopia, os conjunto minimais de raízes são conjuntos unitários.

Caso contrário ponha que $0 \neq r=\#\left\{a_{i} ; a_{i} \neq 0\right\}$ (observe que $r>1$ ) então o conjunto de raízes é como abaixo,

$$
M(f, x)= \begin{cases}\bigsqcup_{j=1}^{\text {tem cardinalidade } r} S_{j}^{n} & , \quad \text { se } x \in W \backslash S^{1} \\ \left(\bigsqcup_{j=1}^{r-2}\left(S^{1} \vee S^{1}\right)_{j}\right) \cup\left(\bigsqcup_{j=1}^{n-r+4} S_{j}^{1}\right), & \text { se } x \in W \backslash S^{2}\end{cases}
$$

Analogamente para o caso em que $n \geq 2$, isto é, uma aplicações especial $f: \mathbb{R} P^{2} \rightarrow W_{n}$ realiza o conjunto minimal de raízes para pontos de $W_{n}$.

Seja o recobrimento universal $p^{\prime}: \widetilde{W} \rightarrow W_{n}$. Sem perda de generalidade podemos supor que $p^{\prime}\left(S_{0}^{2}\right)=e_{0}^{2}$.

Seja $p_{[f]}(t)=a_{0}+\ldots+a_{k} t^{k}$ o polinômio associado a $f$. Se $p_{[f]}(t)$ é o polinômio nulo, então $M(f, x)=\emptyset$ para cada $x \in W_{n}$. Suponha que $p_{[f]}(t)=1$, então o conjunto minimal de raízes é

$$
M(f, x)=\left\{\begin{array}{lll}
\{y\} & \text { se } & x \in e_{0}^{2} \\
\emptyset & & \text { c.c }
\end{array}\right.
$$

Se $k>0$ e $p^{\prime}\left(S_{0}^{2}\right)=e_{0}^{2}$. Para cada $h, h^{\prime} \in\{0, \ldots, n\}$, consideremos $c_{h}$ a cardinalidade do conjunto $\left\{h+j n / h+j n \in\{0, \ldots, k\}, a_{h+j n} \neq 0\right.$ e $\left.j \in \mathbb{N}\right\}$ e $c_{h^{\prime}}$ é a cardinalidade do conjunto $\left\{h^{\prime}+j n / h^{\prime}+j n \in\{0, \ldots, k\}, a_{h^{\prime}+j n}=0\right.$ e $\left.j \in \mathbb{N}\right\}$. Suponha que $k=m n+r$ para alguns $m, r \in \mathbb{N}$. 
Portanto o conjunto minimal de raízes neste caso é

$$
M(f, x)= \begin{cases}\bigsqcup_{j=1}^{\text {tem cardinalidade } c_{h}} S_{j}^{1} & , \quad \text { se } x \in e_{i}^{2} \backslash\left\{x_{i}\right\} \\ \bigsqcup_{j=1}^{m+1} S_{j}^{1} & , \quad \text { se } x \in\left(e_{i}^{1} \backslash\left\{x_{i}, x_{i+1}\right\}\right) \text { e } 1 \leq i \leq r \\ \left(\bigsqcup_{j=1}^{c_{h}}\left(S^{1} \vee S^{1}\right)_{j}\right) \cup\left(\bigsqcup_{j=1}^{c_{h^{\prime}}} S_{j}^{1}\right) & , \quad \text { se } x \in\left(e_{i}^{1} \backslash\left\{x_{i}, x_{i+1}\right\}\right) \text { e } r<i \leq n \\ \left(\bigsqcup_{j=1}^{c_{h}-2}\left(S^{1} \vee S^{1}\right)_{j}\right) \cup\left(\bigsqcup_{j=1}^{c_{h^{\prime}}+2} S_{j}^{1}\right), & \text { se } x=x_{i} \text { e } i \neq 0, r \\ \left(\bigsqcup_{j=1}^{c_{h}-2}\left(S^{1} \vee S^{1}\right)_{j}\right) \cup\left(\bigsqcup_{j=1}^{c_{h^{\prime}}+3} S_{j}^{1}\right), & \text { se } x=x_{r}\end{cases}
$$

\subsection{Raízes de aplicações de $\mathbb{R} P_{g}^{2}$ em $W_{n}$}

Seja uma aplicação $f: \mathbb{R} P_{g}^{2} \rightarrow W$ tal que $\mathbb{R} P_{g}^{2} \simeq T_{k}^{2} \# \mathbb{R} P^{2}$ com $g=2 k+1$ e $k \geq 1$. Considere $\Pi: \widetilde{\mathbb{R} P_{g}^{2}} \rightarrow \mathbb{R} P^{2}$ o recobrimento duplo orientável, onde $\widetilde{\mathbb{R} P_{g}^{2}}$ é como na figura 3.2 .

Na seção 3.2 foi considerado a região fundamental de $\widetilde{\mathbb{R} P_{g}^{2}}$ para fazer modificações, por homotopias, e concluir os resultados. Nesta seção, também, basta utilizar a aplicação $f \circ \Pi$ e a região fundamental de $\widetilde{\mathbb{R} P_{g}^{2}}$ para obter os conjuntos minimais de raízes.

Como vimos na seção 3.2 , uma aplicação especial $h: \mathbb{R} P_{g}^{2} \rightarrow W$ é construída a partir de uma aplicação especial $f: T_{k}^{2} \rightarrow W$. Portanto como uma aplicação especial $h$ realiza o conjunto minimal de raízes para pontos de $W$, então uma aplicação especial $f$ também será uma aplicação que realiza o conjunto minimal de raízes.

Neste caso o conjunto minimal de raízes de $h$ em $x \in W \backslash\{P\}$ é o conjunto minimal de raízes de $f$ em $x \in W \backslash\{P\}$ e o conjunto minimal de raízes de $h$ em $P$ é o conjunto minimal de raízes de $f$ em $P$ união com $S^{1}=\Pi(\partial C N)$.

Seja $p_{[f]}(t)$ o polinômio associado a $f$. Se $p_{[f]}(t)=\mathbb{O}$, então $M(f, x)=\emptyset$ para todo $x \in W$.

Caso $p_{[f]}(t)=1$, então o conjunto $M(f, x)$ tem cardinalidade 1 . 
Caso contrário suponha que $0 \neq r=\#\left\{a_{i} ; a_{i} \neq 0\right\}$ (observe que $r>1$ ) então o conjunto de raízes é como abaixo,

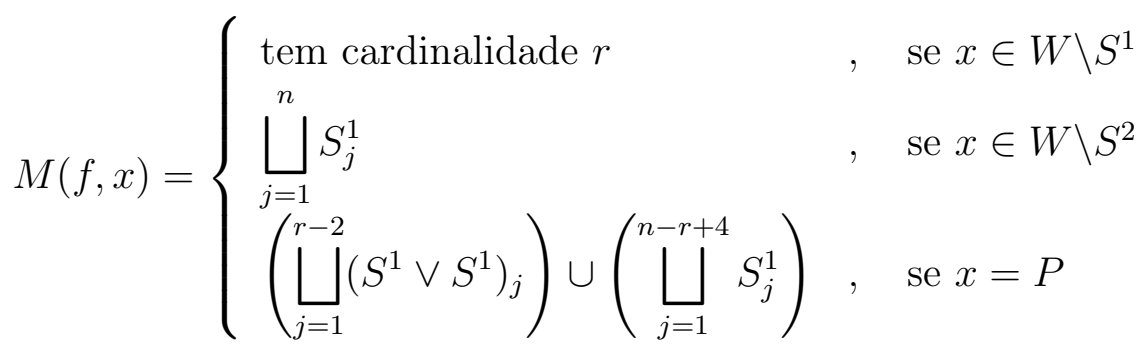

Analogamente para o caso em que $n \geq 2$, isto é, uma aplicações especial $f^{\prime}: T_{k}^{2} \rightarrow W_{n}$ realiza o conjunto minimal de raízes para pontos de $W_{n}$ e consequentemente uma aplicação especial $h^{\prime}: \mathbb{R} P_{g}^{2} \rightarrow W_{n}$ construída a partir de $f^{\prime}$ realiza o conjunto minimal de raízes para os pontos de $W_{n}$.

Seja $p_{\left[h^{\prime}\right]}(t)$ o polinômio associado a $h^{\prime}$. Neste caso o conjunto de raízes é - se $p_{\left[f^{\prime}\right]}(t)=\mathbb{O}$, então $M\left(h^{\prime}, x\right)=\emptyset$ para todo $x \in W$ - se $p_{\left[f^{\prime}\right]}(t)=1$, então $M\left(h^{\prime}, x\right)$ tem cardinalidade 1 .

- caso contrário, desde que o conjunto minimal de raízes de aplicações $h: T_{k}^{2} \rightarrow W$ é igual ao conjunto minimal de raízes de aplicações $h^{\prime}: T_{k}^{2} \rightarrow W_{n}$, segue que o conjunto minimal de raízes de $f^{\prime}: \mathbb{R} P_{g}^{2} \rightarrow W_{n}$ é igual ao conjunto minimal de raízes de aplicações $f: \mathbb{R} P_{g}^{2} \rightarrow W$.

Seja uma aplicação $f: K \rightarrow W$ tal que $K$ é a garrafa de Klein. Considere $\Pi: T^{2} \rightarrow K$ o recobrimento duplo orientável, onde $T^{2}$ é como na figura 3.2 .

Na seção 3.2 foi considerado a região fundamental de $T^{2}$ para fazer modificações, por homotopias, e concluir os resultados. Nesta seção, também, basta utilizar a aplicação $f \circ \Pi$ e a região fundamental de $\widetilde{\mathbb{R} P_{g}^{2}}$ para obter os conjuntos minimais de raízes.

Como vimos na seção [3.2, uma aplicação especial $f: K \rightarrow W$ é construída a partir de uma aplicação $h: F \rightarrow W$. Usando as técnicas da seção 5.1 mostra-se que a aplicação $h$ realiza o conjunto minimal de raízes para pontos de $W$, logo uma aplicação especial $f$ também será uma aplicação que realiza o conjunto minimal de raízes. 
Neste caso o conjunto minimal de raízes de $f$ em $x \in W \backslash S^{1}$ é o conjunto minimal de raízes de $h$ em $x \in W \backslash S^{1}$, o conjunto minimal de raízes de $f$ em $x \in W \backslash S^{2}$ é o conjunto minimal de raízes de $\left.h\right|_{D}$ em $x \in W \backslash S^{2}$ união com $l S^{1}$.

Seja $p_{[f]}(t)=a_{0}+\ldots+a_{n} t^{n}$ o polinômio associado a $f$ e $f_{\pi}\left(\pi_{1}(K)\right) \simeq \mathbb{Z}<l>$. Se $p_{[f]}(t)=\mathbb{O}$ e $l=0$, então $M(f, x)=\emptyset$ para todo $x \in W$.

Se $p_{[f]}(t)=\mathbb{O}$ e $l \neq 0$, então $M(f, x)=\emptyset$ para todo $x \in W \backslash S^{1}$ e $M(f, x)=\bigsqcup_{i=1}^{l} S_{i}^{1}$ para cada $x \in S^{1} \subset W$.

Caso $p_{[f]}(t)=1$ e $l=0$, então $M(f, x)=\emptyset$ para todo $x \in W \backslash S^{2}$ e $M(f, x)$ tem cardinalidade 1 para cada $x \in S^{2} \subset W$.

Caso $\underset{l f f]}{p_{l}}(t)=1$ e $l \neq 0$, então $M(f, x)$ tem cardinalidade 1 para todo $x \in W \backslash S^{1}$, $M(f, x)=\bigsqcup_{i=1}^{l} S_{i}^{1}$ para cada $x \in W \backslash S^{2}$ e $M(f, P)=S^{1} \vee S^{1}$.

Caso contrário ponha que $0 \neq r=\#\left\{a_{i} ; a_{i} \neq 0\right\}$ (observe que $r>1$ ) e $l \neq 0$, então o conjunto minimal de raízes é como abaixo,

$$
M(f, x)= \begin{cases}\text { tem cardinalidade } r & , \text { se } x \in W \backslash S^{1} \\ \bigsqcup_{j=1}^{n+l} S_{j}^{1} & , \text { se } x \in W \backslash S^{2} \\ \left(\bigsqcup_{j=1}^{r-1}\left(S^{1} \vee S^{1}\right)_{j}\right) \cup\left(\bigsqcup_{j=1}^{n-r+3+l-1} S_{j}^{1}\right) & , \text { se } x=P\end{cases}
$$

Analogamente para o caso em que $n \geq 2$, isto é, uma aplicações especial $h: T^{2} \rightarrow W_{n}$ realiza o conjunto minimal de raízes para pontos de $W_{n}$ e consequentemente a aplicação especial $f: K \rightarrow W_{n}$ construída a partir de $f$ realiza o conjunto minimal de raízes para os pontos de $W_{n}$.

Seja $p_{[f]}(t)=a_{0}+\ldots+a_{k} t^{k}$ o polinômio associado a $f$ e $f_{\pi}\left(\pi_{1}(K)\right) \simeq \mathbb{Z}<l>$. Neste caso o conjunto de raízes é

- se $p_{[f]}(t)=\mathbb{O}$ e $l=0$, então $M(f, x)=\emptyset$ para todo $x \in W$.

- se $p_{[f]}(t)=\mathbb{O}$ e $l \neq 0$, então $M(f, x)=\emptyset$ para todo $x \in W \backslash S^{1}$ e $M(f, x)=\bigsqcup_{i=1}^{l} S_{i}^{1}$ para cada $x \in S^{1} \subset W$.

- caso $p_{[f]}(t)=1$ e $l=0$, então $M(f, x)=\emptyset$ para todo $x \in W \backslash S^{2}$ e $M(f, x)$ tem 
cardinalidade 1 para cada $x \in S^{2} \subset W$.

- caso $p_{[f]}(t)=1$ e $l \neq 0$, então $M(f, x)$ tem cardinalidade 1 para cada $x \in W \backslash S^{1}$, $M(f, x)=\bigsqcup_{i=1}^{l} S_{i}^{1}$ para todo $x \in W \backslash S^{2}$ e $M(f, P)=S^{1} \vee S^{1}$.

Caso contrário, desde que o conjunto minimal de raízes de aplicações $\left.f\right|_{\Pi(D)}: \Pi(D) \rightarrow$ $W_{n}$ é igual ao conjunto minimal de raízes de aplicações $f^{\prime}: D \rightarrow W_{n}$, segue que o conjunto minimal de raízes de $f: K \rightarrow W_{n}$ é:

- para $x \in W_{n} \backslash S^{1}, M(f, x)=M\left(f^{\prime}, x\right)$.

- para $x \in e_{i}^{1} \backslash\left\{x_{i}, x_{i+1}\right\}, M(f, x)=M\left(f^{\prime}, x\right) \bigcup \bigsqcup_{i=1}^{l} S_{i}^{1}$.

- ponha que $k=\underset{l}{j n}+m$, onde $0 \leq m<n$. Logo para $x=x_{i}$ tal que $i \neq m$, $M(f, x)=M\left(f^{\prime}, x\right) \bigcup \bigsqcup_{i=1} S_{i}^{1}$

- para $x=x_{m}, M(f, x)=\left(M\left(f^{\prime}, x\right) \backslash S^{1}\right) \bigcup S^{1} \vee S^{1} \bigsqcup_{i=1}^{l-1} S_{i}^{1}$.

Por fim, sejam $f: \mathbb{R} P_{g}^{2} \rightarrow W$ tal que $g=2 k \operatorname{com} k>1$ e $\Pi: T_{2+1} \rightarrow W$ o recobrimento duplo orientável.

Da mesma maneira que antes, as aplicações especiais realizam o conjunto minimal de raízes.

Uma aplicação especial é construída a partir de uma aplicação $h: F \rightarrow W$, onde $F$ é a região fundamental de $T_{2 k+1}$. Na construção da aplicação especial são identificadas duas circunferências de $F$ para obter uma aplicação $h^{\prime}: T_{k+1} \rightarrow W$ para construir $f$.

Portanto para $x \in W, M(f, x)$ é igual $M\left(h^{\prime}, x\right)$.

Analogamente para o caso em que $n \geq 2$, isto é, uma aplicações especial $f: \mathbb{R} P_{g}^{2} \rightarrow W_{n}$ realiza o conjunto minimal de raízes para pontos de $W_{n}$ e para cada $x \in W_{n}, M(f, x)$ é igual $M\left(h^{\prime}, x\right)$, onde $h^{\prime}: T_{k+1} \rightarrow W_{n}$ é uma aplicação especial usada para construir a aplicação $f$. 


\section{Apêndice}

\section{Homologia com Coeficientes Locais}

Apresentaremos uma introdução de homologia com coeficientes locais que foi usada ao longo do trabalho, para mais detalhes ver livro [13].

Dado um grupo $\pi$, denotaremos por $\mathbb{Z} \pi$ o seu anel de grupo.

Sejam $A$ um grupo abeliano e um homomorfismo de grupos $\rho: \pi \rightarrow \operatorname{Aut}_{\mathbb{Z}}(A)$, onde $A u t_{\mathbb{Z}}(A)=\{h: A \rightarrow A \mid h$ é automorfismo $\}$. O homomorfismo $\rho$ induz em $A$ uma estrutura de $\mathbb{Z} \pi$-módulo a esquerda pela seguinte ação

$$
\left(\sum_{g \in \pi} m_{g} g\right) a=\sum_{g \in \pi} m_{g} \rho(g)(a) .
$$

Reciprocamente se $A$ é um módulo a esquerda sobre o anel $\mathbb{Z} \pi$, então existe um homomorfismo

$$
\rho: \pi \rightarrow \operatorname{Aut}_{\mathbb{Z}}(A)
$$

dado por $\rho(g)(a)=g a$, onde $g a$ é a multiplicação de $a \in A$ por $g \in \pi$.

Definição 6.2.1. Sejam E, B espaços topológicos, A grupo abeliano com a topologia discreta e $G$ um subgrupo de $\operatorname{Aut}_{\mathbb{Z}}(A)$. Então um recobrimento $p: E \rightarrow B$ com fibra $A$ e grupo estrutural $G$ é um sistema de coeficientes locais sobre B. Além disso, cada fibra $p^{-1}(b)$ é isomorfa a $A$.

Definição 6.2.2. Seja $p: E \rightarrow B$ um $G$-fibrado principal. Suponha que $G$ atua a esquerda sobre um espaço $F$, isto é, é dada uma ação $G \times F \rightarrow F$. A construção de Borel

$$
E \times{ }_{G} F
$$


é dado pelo espaço quociente $\frac{E \times F}{\sim}$, onde $(x, f) \sim\left(x g, g^{-1} f\right)$.

Denotemos a classe de equivalência de $(x, f)$ por $[x, f] \in E \times_{G} F$. Definimos a aplicação $q: E \times{ }_{G} F \rightarrow B$ por $q([x, f])=p(x)$.

Definição 6.2.3. Se $p: E \rightarrow B$ é um G-fibrado principal e $G$ atua sobre $F$, então

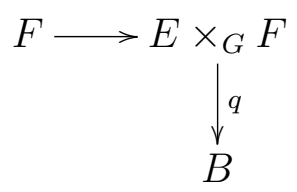

é um fibrado sobre $B$ com fibra $F$ e grupo estrutural $G$, onde $q([x, f])=p(x)$. A aplicação $q: E \times \times_{G} F \rightarrow B$ é chamada fibrado associado ao fibrado principal $p: E \rightarrow B$ via a ação de $G$ sobre F.

Lema 6.2.1. Todo sistema de coeficientes locais sobre um espaço B conexo por caminhos e semi-localmente simplesmente conexo é da forma

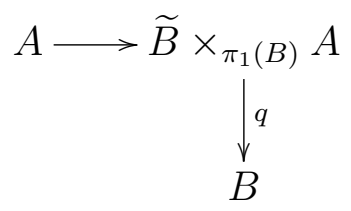

isto é, é o fibrado associado ao $\pi_{1}(B)$-fibrado principal dado pelo recobrimento universal $\widetilde{B}$ de $B$, onde a ação é dada por um homomorfismo $\pi_{1}(B) \rightarrow \operatorname{Aut}_{\mathbb{Z}}(A)$.

Seja $X$ um espaço topológico conexo, localmente conexo por caminhos e semilocalmente simplesmente conexo com recobrimento universal $\tilde{X}$. Daqui por diante $\pi=\pi_{1}(X)$. Para definir homologia com coeficientes locais daremos uma estrutura de $\mathbb{Z} \pi$-módulo aos complexos singulares $S_{*}(\widetilde{X})$, onde $S_{*}(\widetilde{X})$ é o grupo abeliano livre gerado por $\left\{\delta: \Delta^{*} \rightarrow\right.$ $\widetilde{X} \mid \delta$ é contínua\} e $\partial$ é o operador bordo usual.

Seja $\tilde{X} \rightarrow X$ o recobrimento universal de $X$ com a $\pi$-ação usual a direita obtida por identificação de $\pi$ com o grupo de transformações de recobrimento.

A ação de um elemento $g \in \pi$ sobre um simplexo singular $\delta: \Delta^{k} \rightarrow \widetilde{X}$ é o simplexo singular $g \delta$ definido como a composição de $\delta$ e a transformação recobrimento $g: \widetilde{X} \rightarrow \widetilde{X}$. 
Estendendo esta ação por linearidade para $\mathbb{Z} \pi$, tem-se que o complexo de cadeias do recobrimento universal é constituído por $\mathbb{Z} \pi$-módulos livres a direita e os operadores bordos são $\mathbb{Z} \pi$ homomorfismos.

Definição 6.2.4. Seja um espaço topológico conexo, localmente conexo por caminhos e semilocalmente simplesmente conexo com recobrimento universal $\tilde{X}$. Dado um $\mathbb{Z} \pi$-módulo $A$, então o produto tensorial

$$
S_{*}(X ; A)=S_{*}(\tilde{X}) \otimes_{\mathbb{Z} \pi} A
$$

é um complexo de cadeias cuja homologia é chamada homologia de X com coeficientes locais em $A$ e é denotado por $H_{*}(X ; A)$.

A estrutura de $\mathbb{Z} \pi$-módulo de $A$ é determinada, como vimos anteriormente, por um homomorfismo $\rho: \pi_{1}(X) \rightarrow \operatorname{Aut}_{\mathbb{Z}}(A)$. Ás vezes para enfatizar este homomorfismo colocaremos um subíndice $\rho$ em $A$ e denotaremos $H_{*}\left(X ; A_{\rho}\right)$ para homologia com coeficientes em $A$.

Definição 6.2.5. Sejam $p: E \rightarrow B$ um fibrado com fibra $F$ e grupo estrutural $G$. Dada uma aplicação $f: B^{\prime} \rightarrow B$ o "pullback" de $p: E \rightarrow B$ por $f$ é o espaço

$$
f^{*}(E)=\left\{\left(b^{\prime}, e\right) \in B^{\prime} \times E \mid p(e)=f\left(b^{\prime}\right)\right\} .
$$

Se considerarmos $q: f^{*}(E) \rightarrow B^{\prime}$ como sendo a restrição da projeção $E \times B^{\prime} \rightarrow B^{\prime}$ a $f^{*}(E)$, então $q$ é um fibrado com fibra $F$ e grupo estrutural $G$ com o seguinte diagrama comutativo

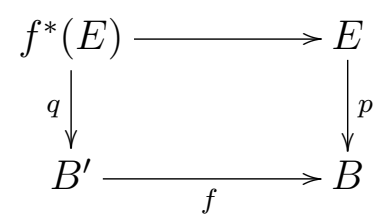

Observe que dados uma aplicação $f: B^{\prime} \rightarrow B$ e um sistema local em $B$, usando o "pullback" tem-se um sistema local sobre $B$ '. 


\section{Sequência de Puppe-Barrat}

Apresentamos alguns resultados de caracterização de classes de homotopia, a referência principal é [21].

\section{Cone, suspensão e produto smash}

Para efeito de notação colocamos alguns conceitos e resultados.

Definição 6.2.6. O produto smash $(X \wedge Y, *)$ entre dois espaços topológicos pontuados $\left(X, x_{0}\right)$ e $\left(Y, y_{0}\right)$ é o quociente

$$
\frac{X \times Y}{X \vee Y}
$$

com o ponto base $*=p(X \vee Y)$, onde $\vee$ denota o wedge e $p: X \times Y \rightarrow X \wedge Y$ é a projeção tal que $p(x, y) \in X \wedge Y$ é denotado por $[x, y]$.

Teorema 6.2.1. Sejam $\left(X, x_{0}\right),\left(Y, y_{0}\right),\left(Z, z_{0}\right)$ espaços topológicos pontuados, tais que $X, Z$ são Hausdorff e $Z$ é localmente compacto, então existe uma equivalência de homotopia

$$
A:\left[(Z \wedge X, *) ;\left(Y, y_{0}\right)\right] \rightarrow\left[\left(X, x_{0}\right) ;\left(Y, y_{0}\right)^{\left(Z, z_{0}\right)}, f_{0}\right]
$$

definida por $A([f])=[\hat{f}]$, onde $\hat{f}: X \rightarrow Y^{Z}$ é dada por $(\hat{f}(x))(z)=f([z, x])$.

A prova deste teorema pode ser encontrada em [21]. Este resultado implica que podemos identificar os espaços $\left[(Z \wedge X, *) ;\left(Y, y_{0}\right)\right]$ e $\left[\left(X, x_{0}\right) ;\left(Y, y_{0}\right)^{\left(Z, z_{0}\right)}, f_{0}\right]$, onde $f_{0}:\left(Z, z_{0}\right) \rightarrow$ $\left(Y, y_{0}\right)$ é a aplicação constante e este resultado será usado mais adiante.

Definição 6.2.7. Se $\left(X, x_{0}\right)$ é um espaço topológico pontuado, defini-se a suspensão pontuada $(S X, *)$ de $X$, pelo produto smash de $X$ e da 1-esfera, isto é, $\left(S^{1} \wedge X, *\right)$. Além disso, dada uma função contínua entre espaços pontuados $f:\left(X, x_{0}\right) \rightarrow\left(Y, y_{0}\right)$ podemos definir a aplicação Sf suspensão de $f$, por

$$
S f=1 \wedge f:\left(S^{1} \wedge X, *\right) \rightarrow\left(S^{1} \wedge Y, *\right)
$$


Observação: A operação de suspensão é um funtor, chamado funtor suspensão.

Definição 6.2.8. Considere um espaço topológico pontuado $\left(X, x_{0}\right)$. Então o cone pontuado $(C X, *)$ é o produto smash $(I \wedge X, *)$, onde o ponto base de $I$ é o 0 . Logo $C X$ é o quociente

$$
C X=\frac{I \times X}{(\{0\} \times X) \cup\left(I \times\left\{x_{0}\right\}\right)} .
$$

Por exemplo se considerar o espaço pontuado $X=S^{1} x_{0} \in S^{1}$. Então o $\left(C S^{1}, *\right) \cong D^{2}$.

Nós usaremos a notação $[t, x]$ para a imagem pela projeção de $(t, x) \in I \times X$ em $C X$. A aplicação $i: X \rightarrow C X$ dada por $i(x)=[1, x], x \in X$, é um homeomorfismo com sua imagem $\operatorname{Im} i$. Dessa forma podemos identificar o espaço $X$ com $\operatorname{Im} i$ e assim olhar $X$ como um subespaço de $C X$. Logo $C X / X \cong S X$.

Definição 6.2.9. Dados uma função contínua $f:\left(X, x_{0}\right) \rightarrow\left(Y, y_{0}\right)$ e $C X$ o cone de $X$, considere o espaço $Y \cup_{f} C X$ obtido de $Y \vee C X$ identificando os pontos $[1, x] \in C X$ com os pontos $f(x)$ para todo $x \in X$. Intuitivamente colamos a base do cone $C X$ com $Y$ através de $f$.

Considere $X=S^{1}, Y=S^{1} \vee S^{1}$ e $f:\left(S^{1}, x_{0}\right) \rightarrow\left(S^{1} \vee S^{1}, y_{0}\right)$ dada por $f\left(S^{1}\right)=$ $a b a^{-1} b^{-1}$, onde $a$ significa que a imagem de $S^{1}$ pela $f$ recobre $S^{1} \vee *$ injetivamente em uma direção e $a^{-1}$ recobre injetivamente em outra direção, analogamente $b$ e $b^{-1}$ recobre $* \vee S^{1}$. Então $Y \cup_{f} C X \cong T^{2}$.

Os próximos dois resultados podem serem encontrados em [21] e resultará numa equivalência de homotopia que será usado posteriormente.

Lema 6.2.2. Para qualquer função contínua entre espaços topológicos $f:\left(X, x_{0}\right) \rightarrow$ $\left(Y, y_{0}\right)$ a projeção

$$
q:\left(Y \cup_{f} C X\right) \cup_{j} C Y \rightarrow \frac{\left(Y \cup_{f} C X\right) \cup_{j} C Y}{C Y}
$$

é uma equivalência de homotopia, onde $j: Y \rightarrow Y \cup_{f} C X$ é a inclusão.

Proposição 6.2.1. Se $A \subset X$ é um subespaço, então $\frac{X \cup_{i} C A}{C A}$ é homeomorfo a $\frac{X}{A}$, onde $i: A \rightarrow X$ é a inclusão.

Em particular temos $\frac{\left(Y \cup_{f} C X\right) \cup_{j} C Y}{C Y} \cong \frac{Y \cup_{f} C X}{Y} \cong \frac{C X}{X} \cong S X$. 


\section{Sequência de Puppe-Barrat}

Nesta seção será introduzido a sequência de Puppe-Barrat, que pode ser encontrada em [21]. Os estudos desta sequência foi iniciado por Michael G. Barratt em [3] e [4] e propriedades e alguns resultados sobre a sequência de Puppe foi feita por Dieter Puppe em [18]. A construção desta sequência fornece uma ação de grupo. A sequência junto com a ação caracterizará um elemento $f \in[S, W]$ explicitando geometricamente como é a aplicação. Segue abaixo, alguns conceitos e resultados necessários para definir a sequência.

Relembraremos alguns conceitos de álgebra e definiremos a sequência exata de PuppeBarrat.

Definição 6.2.10. Um grupo é um conjunto pontuado $(G, e)$ com uma multiplicação $\mu: G \times G \rightarrow G$ e uma inversa $\nu: G \rightarrow G$ tais que os seguintes diagramas comutam:

1. e é uma identidade nos dois lados;

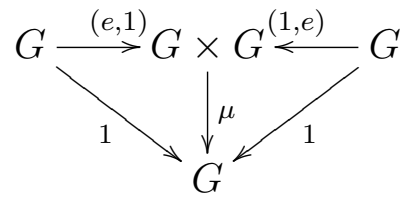

2. associatividade;

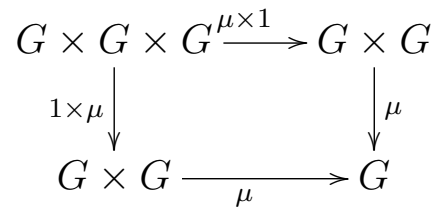

3. inversa;

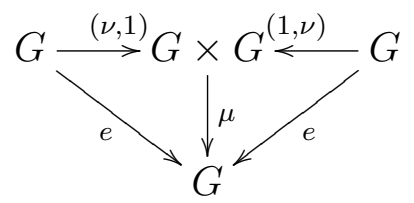

onde $e: G \rightarrow G$ é a aplicação constante $e(g)=e$ para todo $g \in G,(e, 1)(g)=(e, g)$ para todo $g \in G$. 
Além disso, $G$ é chamado comutativo ou abeliano se o seguinte diagrama comuta

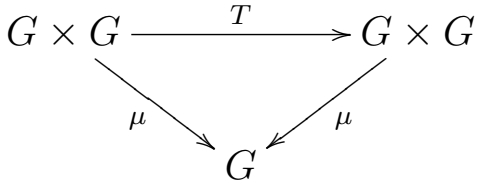

onde $T: G \times G \rightarrow G \times G$ é dada por $T\left(g_{1}, g_{2}\right)=\left(g_{2}, g_{1}\right)$ para todo $\left(g_{1}, g_{2}\right) \in G \times G$.

Motivado pela definição de grupo foi feita a seguinte definição.

Definição 6.2.11. Um $H$-espaço é um espaço pontuado $\left(K, k_{0}\right)$ com uma aplicação multiplicação $\mu: K \times K \rightarrow K$ tal que vale as seguintes propriedades:

1. $k_{0}: K \rightarrow K$ é uma identidade homotópica, isto é,o diagrama

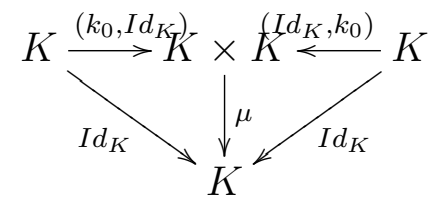

comuta homotopicamente, isto é, $\mu \circ\left(I d_{K}, k_{0}\right) \cong I d_{K} \cong \mu \circ\left(k_{0}, I d_{K}\right)$.

2. $\mu$ é associativa homotópica, isto é, o diagrama

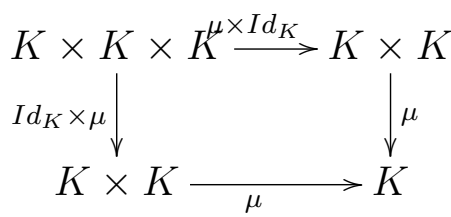

o diagrama acima comuta homotopicamente: $\mu \circ\left(\mu \times I d_{K}\right) \cong \mu \circ\left(I d_{K} \times \mu\right)$.

3. $\nu:\left(K, k_{0}\right) \rightarrow\left(K, k_{0}\right)$ é inversa homotópica:

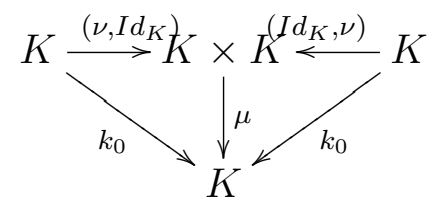

o diagrama acima comuta homotópicamente: $\mu \circ\left(\nu, I_{K}\right) \cong k_{0} \cong \mu \circ\left(\operatorname{Id}_{K}, \nu\right)$. 
4. $\mu$ é comutativo homotópica:

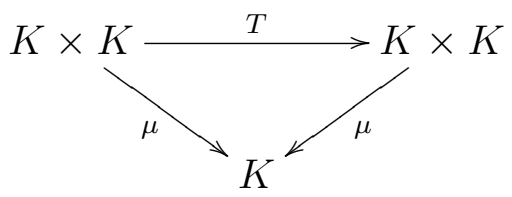

o diagrama acima comuta homotopicamente: $\mu \circ T \cong \mu$, onde $T: K \times K \rightarrow K \times K$

é dada por $T\left(k_{1}, k_{2}\right)=\left(k_{2}, k_{1}\right)$ para todo $\left(k_{1}, k_{2}\right) \in K \times K$.

Um H-grupo é um $H$-espaço $\left(K, k_{0}\right)$ com multiplicação associativa homotópica $\mu$ e inversa homotópica $\nu$. Para a simplificar notação não foi usado ponto base, mas todas as aplicações na definição são relativas ao ponto base.

A partir desta definição temos a seguinte proposição:

Proposição 6.2.2. Se $\left(K, k_{0}\right)$ é um H-grupo com multiplicação $\mu^{\prime}$ e inversa homotópica $\nu^{\prime}$, então para qualquer espaço topológico pontuado $\left(X, x_{0}\right)$ o conjunto

$$
\left[\left(X, x_{0}\right) ;\left(K, k_{0}\right)\right]
$$

tem uma estrutura de grupo se definirmos o produto $[f][g]$ pela classe de homotopia da seguinte composição:

$$
X \stackrel{\Delta}{\rightarrow} X \times X \stackrel{f \times g}{\rightarrow} K \times K \stackrel{\mu}{\rightarrow} K
$$

onde $\Delta$ é dada por $\Delta(x)=(x, x)$. A identidade do grupo é a classe $\left[k_{0}\right]$ da função constante e a inversa é dada por $[f]^{-1}=[\nu \circ f]$. Se $\mu^{\prime}$ é homotópica comutativa, então $\left[\left(X, x_{0}\right) ;\left(K, k_{0}\right)\right]$ é abeliano. Toda função $f:\left(X, x_{0}\right) \rightarrow\left(Y, y_{0}\right)$ induz um homomorfismo de grupos

$$
f^{*}:\left[\left(Y, y_{0}\right) ;\left(K, k_{0}\right)\right] \rightarrow\left[\left(X, x_{0}\right) ;\left(K, k_{0}\right)\right]
$$

Assim como foi feito a construção a partir de um $H$-grupo, pode ser feito uma construção "dualizando" os espaço e as função, por exemplo será feito tomando o espaço $K \vee K$ ao invés de $K \times K$.

Definição 6.2.12. Um $H$-cogrupo é um espaço topológico pontuado $\left(K, k_{0}\right)$ junto com um função contínua comultiplicação $\mu^{\prime}: K \rightarrow K \vee K$ tal que: 
1. $k_{0}$ é a homotopia identidade, isto é, o diagrama

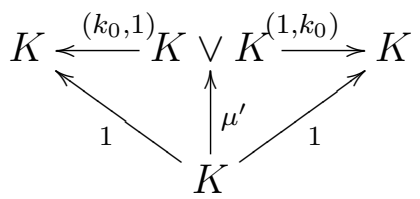

comuta homotopicamente, onde $\left(k_{0}, 1\right)\left(k, k_{0}\right)=k_{0} e\left(k_{0}, 1\right)\left(k_{0}, k\right)=k$ para todo $k \in K$.

2. $\mu^{\prime}$ é associativa homotópica, isto é, o diagrama

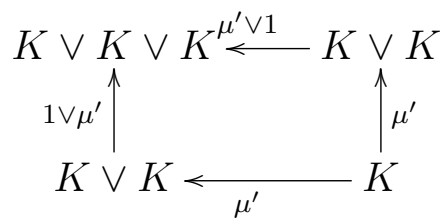

comuta homotopicamente.

3. $K$ tem uma inversa homotópica $\nu^{\prime}: K \rightarrow K$ tal que o diagrama

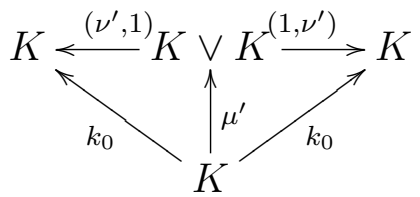

comuta homotopicamente.

Além disso, $K$ é um $H$-cogrupo comutativo homotópico se o diagrama

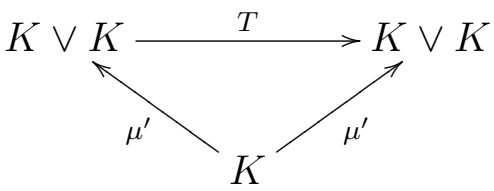

comuta homotopicamente.

Um exemplo de $H$-cogroupo é $\left(S X, x_{0}\right)$ para um espaço topológico $X$. A aplicação comultiplicação $\mu^{\prime}$ é definida por 


$$
\mu^{\prime}([t, x])= \begin{cases}\left([2 t, x], x_{0}\right) & \text { se } 0 \leq t \leq \frac{1}{2} \\ \left(x_{0},[2 t-1, x]\right) & \text { se } \frac{1}{2} \leq t \leq 1\end{cases}
$$

A homotopia inversa $\nu^{\prime}: S X \rightarrow S X$ é dada por $\nu^{\prime}([t, x])=[1-t, x]$, para todo $t \in I$, $x \in X$.

Proposição 6.2.3. Se $\left(K, k_{0}\right)$ é um $H$-cogrupo com comultiplicação $\mu^{\prime}$ e inversa homotópica $\nu^{\prime}$, então para qualquer espaço topológico pontuado $\left(X, x_{0}\right)$ o conjunto

$$
\left[\left(K, k_{0}\right) ;\left(X, x_{0}\right)\right]
$$

tem uma estrutura de grupo definida por; $[f][g]$ é a classe de homotopia da seguinte composição:

$$
K \stackrel{\mu^{\prime}}{\rightarrow} K \vee K \stackrel{f \vee g}{\rightarrow} X \vee X \stackrel{\Delta^{\prime}}{\rightarrow} X
$$

onde $\Delta^{\prime}$ é dada por $\Delta^{\prime}\left(x, x_{0}\right)=x=\Delta^{\prime}\left(x_{0}, x\right)$. A identidade do grupo é a classe $\left[x_{0}\right]$ da função constante e a inversa é dada por $[f]^{-1}=\left[f \vee \nu^{\prime}\right]$. Se $\mu^{\prime}$ é homotópica comutativa, então $\left[\left(K, k_{0}\right) ;\left(X, x_{0}\right)\right]$ é abeliano. Toda função $f:\left(X, x_{0}\right) \rightarrow\left(Y, y_{0}\right)$ induz um homomorfismo de grupos

$$
f_{*}:\left[\left(K, k_{0}\right) ;\left(X, x_{0}\right)\right] \rightarrow\left[\left(K, k_{0}\right) ;\left(Y, y_{0}\right)\right] .
$$

Dado um espaço topológico $X$, denotemos $S^{n} X$ pela suspensão do espaço $S^{n-1} X$, para $n \geq 1$ e $S^{0} X=X$. Então vale os seguintes três resultados e suas provas podem ser encontradas em [21].

Proposição 6.2.4. Dado um espaço topológico X. Então $S^{n} X$ é um H-cogrupo comutativo homotópico, para $n \geq 2$.

Lema 6.2.3. Para todo $n \geq 0$ tem-se $S^{1} \wedge S^{n}$ é homeomorfo a $S^{n+1}$, onde $S^{i}$ é a esfera de dimensão $i$.

Corolário 6.2.1.1. Para todo espaço topológico $X$ e $n \geq 0$ tem-se que $S^{n} X$ é homeomorfo $a S^{n} \wedge X$. 
Definição 6.2.13. Um grupo $G$ atua (à esquerda) sobre um conjunto $A$ se existe uma função $\alpha: G \times A \rightarrow A$ tal que os seguintes diagramas são comutativos:
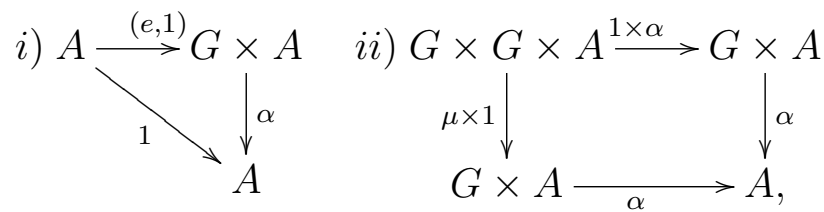

onde $\mu: G \times G \rightarrow G$ é a função multiplicação de $G$ e $(e, 1)(x)=(e, x)$ para todo $x \in A$. As órbitas da ação são os conjuntos $G x=\{g x: g \in G\}$ para $x \in A$.

Definição 6.2.14. Um $H$-cogrupo $\left(K, k_{0}, \mu^{\prime}\right)$ coatua sobre um espaço $\left(X, x_{0}\right)$ se existe um função coação $\alpha^{\prime}: X \rightarrow K \vee X$ tais que os seguintes diagramas comutam homotopicamente
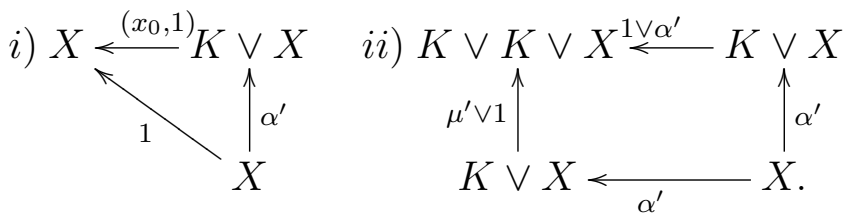

Proposição 6.2.5. Se o $H$-cogrupo $\left(K, k_{0}, \mu^{\prime}\right)$ coatua sobre o espaço $\left(X, x_{0}\right)$ com a função coação $\alpha^{\prime}$, então para todo espaço $\left(W, w_{0}\right)$ existe uma ação natural do grupo $\left[\left(K, k_{0}\right) ;\left(W, w_{0}\right)\right]$ sobre o conjunto $\left[\left(X, x_{0}\right) ;\left(W, w_{0}\right)\right]$ dada por

$$
\alpha([f],[g])=\left[\Delta^{\prime} \circ(f \vee g) \circ \alpha^{\prime}\right],
$$

onde $f:\left(K, k_{0}\right) \rightarrow\left(W, w_{0}\right)$ e $g:\left(X, x_{0}\right) \rightarrow\left(W, w_{0}\right)$ são quaisquer.

Com estas definições e resultados definiremos a principal aplicação coação que será importante para os resultados da próxima subseção.

Dados dois espaços topológicos pontuados $\left(X, x_{0}\right),\left(Y, y_{0}\right)$ e uma função contínua $f$ : $\left(X, x_{0}\right) \rightarrow\left(Y, y_{0}\right)$, constrói-se a aplicação

$$
\alpha^{\prime}: Y \cup_{f} C X \rightarrow S X \vee\left(Y \cup_{f} C X\right)
$$

definida por

$$
\alpha^{\prime}(y)=(*, y)
$$




$$
\alpha^{\prime}([t, x])= \begin{cases}([2 t, x], *) & \text { se } 0 \leq t \leq \frac{1}{2} \\ (*,[2 t-1, x]) & \text { se } \frac{1}{2} \leq t \leq 1\end{cases}
$$

geometricamente temos
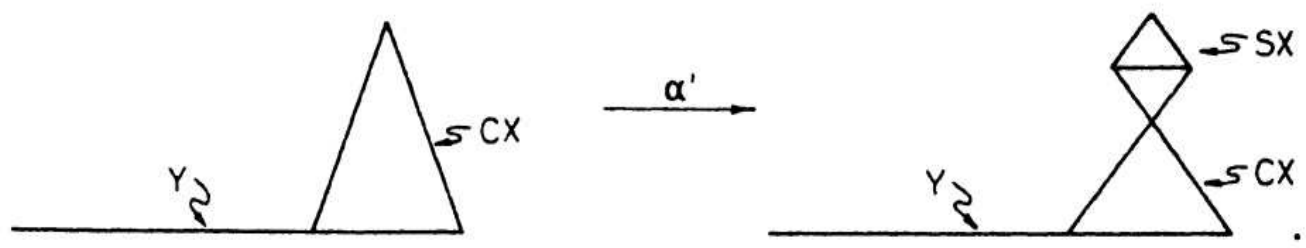

Lema 6.2.4. A função $\alpha^{\prime}$ definida acima é uma coação. Além disso, considere as inclusões $j: Y \rightarrow Y \cup_{f} C X$ e $k: Y \cup_{f} C X \rightarrow\left(Y \cup_{f} C X\right) \cup_{j} C Y$ e a equivalência de homotopia $\bar{q}:\left(Y \cup_{f} C X\right) \cup_{j} C Y \rightarrow S X$. Defina $k^{\prime}=\bar{q} \circ k$. Então $\alpha^{\prime}$ tem as seguintes propriedades: i) $\alpha^{\prime} \circ j=(*, j)$, ii) $\mu^{\prime} \circ k^{\prime}=\left(1 \vee k^{\prime}\right) \circ \alpha^{\prime}$.

Exemplo 1. Consideremos $X=S^{1}, Y=S^{1} \vee S^{1}$ e $f: X \rightarrow Y$ função continua tal que $C S^{1} \cup_{f} S^{1} \vee S^{1}=T^{2}$. Então $\alpha^{\prime}: Y \cup_{f} C X \rightarrow S X \vee\left(Y \cup_{f} C X\right)$ é da seguinte forma;

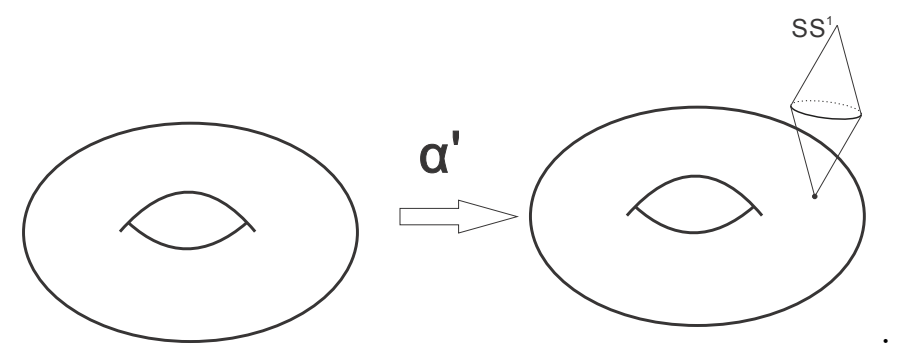

isto significa que a coação $\alpha^{\prime}$ "pinça" uma $S^{1}$ (bordo de um disco $D^{2}$ ) a um ponto, dando origem à uma $S^{2}=S S^{1}$ colada ao toro por um "ponto".

Na próxima subseção será descrita a ação de $\left[\left(S^{2}, s_{0}\right) ;\left(W, w_{0}\right)\right]$ sobre o conjunto $\left[\left(S, x_{0}\right) ;\left(W, w_{0}\right)\right]$ dada por esta $\alpha^{\prime}$.

Dadas duas aplicações $f_{1}, f_{2}: Y \cup_{f} C X \rightarrow W$ tal que $\left.f_{1}\right|_{Y}=\left.f_{2}\right|_{Y}$ podemos definir

$$
d\left(f_{1}, f_{2}\right): S X \rightarrow W
$$


por

$$
d\left(f_{1}, f_{2}\right)([t, x])=\left\{\begin{array}{lll}
f_{1}([2 t, x]) & \text { se } & 0 \leq t \leq \frac{1}{2} \\
f_{2}([2-2 t, x]) & \text { se } & \frac{1}{2} \leq t \leq 1
\end{array}\right.
$$

Lema 6.2.5. Para quaisquer aplicações $f_{1}, f_{2}: Y \cup_{f} C X \rightarrow W$ tais que $\left.f_{1}\right|_{Y}=\left.f_{2}\right|_{Y}$ tem-se

$$
\left[d\left(f_{1}, f_{2}\right)\right]\left[f_{2}\right]=\left[f_{1}\right]
$$

Teorema 6.2.2. Sejam $f:\left(X, x_{0}\right) \rightarrow\left(Y, y_{0}\right)$ uma função contínua entre espaços topológicos pontuados, $\left(W, w_{0}\right)$ um espaço topológico pontuado e as inclusões $j: Y \rightarrow$ $Y \cup_{f} C X$ e $k: Y \cup_{f} C X \rightarrow\left(Y \cup_{f} C X\right) \cup_{j} C Y$. Considere a equivalência de homotopia $\bar{q}:\left(Y \cup_{f} C X\right) \cup_{j} C Y \rightarrow S X$. Então existe uma ação natural do grupo $\left[(S X, *) ;\left(W, w_{0}\right)\right]$ sobre o conjunto $\left[\left(Y \cup_{f} C X, *\right) ;\left(W, w_{0}\right)\right]$ tal que na sequência exata abaixo, conhecida como sequência de Puppe-Barrat,

$$
[X ; W] \stackrel{f^{*}}{\longleftarrow}[Y ; W] \stackrel{j^{*}}{\longleftarrow}\left[Y \cup_{f} C X ; W\right] \stackrel{k^{* *}}{\longleftarrow}[S X ; W] \stackrel{S f^{*}}{\longleftarrow}[S Y ; W]
$$

vale o seguinte:

i) para $x_{1}, x_{2} \in\left[Y \cup_{f} C X ; W\right]$, temos $j^{*}\left(x_{1}\right)=j^{*}\left(x_{2}\right)$ se, e somente se, existe $\theta \in[S X ; W]$ tal que $\theta x_{2}=x_{1}$;

ii) para $y_{1}, y_{2} \in[S X ; W]$ temos $k^{\prime *}\left(y_{1}+y_{2}\right)=y_{1} \cdot k^{\prime *}\left(y_{2}\right)$;

iii) para $y_{1}, y_{2} \in[S X ; W]$ temos $k^{\prime *}\left(y_{1}\right)=k^{\prime *}\left(y_{2}\right)$, se e somente se, existe um $\gamma \in[S Y ; W]$ com $y_{2}=y_{1}+S f^{*}(\gamma)$;

iv) $k^{\prime}(y)=y \cdot\left[w_{0}\right]$ para todo $y \in[S X ; W]$.

A prova deste teorema e dos dois lemas anteriores podem ser encontrados em [21] nos capítulos 1 e 2 .

\subsection{A sequência de Puppe-Barrat no caso $\left[T^{2} ; W\right]$ e al- guns resultados}

Usando os resultados das seções anteriores entre outros, será feito uma descrição geométrica da ação de $\left[S^{2} ; W\right]$ sobre $\left[T^{2} ; W\right]$ e utilizando a sequência de Puppe-Barrat, as 
classes de homotopia de aplicações de $T^{2}$ em $W$ serão caracterizadas. Para uma melhor didática as classes de homotopia de aplicações serão dividas em vários casos.

Seguindo as notações da seção anterior, temos os $X=S^{1}, Y=S^{1} \vee S^{1}$. Considere $f: X \rightarrow Y$ a função contínua tal que $D^{2} \cup_{f} S^{1} \vee S^{1}=T^{2}$ e $j: Y \rightarrow S^{1} \cup_{f} C\left(S^{1}\right)$ é a inclusão natural.

Construindo a sequência de Puppe-Barrat para estes espaços temos:

$$
\begin{gathered}
{\left[S^{1} ; W\right] \stackrel{f^{*}}{\longleftarrow}\left[S^{1} \vee S^{1} ; W\right] \stackrel{j^{*}}{\longleftarrow}\left[S^{1} \vee S^{1} \cup_{f} C\left(S^{1}\right) ; S^{2} \vee T^{2}\right] \stackrel{k^{\prime *}}{\longleftarrow}} \\
\stackrel{k^{\prime *}}{\longleftarrow}\left[S\left(S^{1}\right) ; W\right] \stackrel{S f^{*}}{\longleftarrow}\left[S\left(S^{1} \vee S^{1}\right) ; W\right]
\end{gathered}
$$

Pelas definições de suspensão e cone, temos $S\left(S^{1}\right)=S^{2}$ e $\left(S^{1} \vee S^{1}\right) \cup_{f} C\left(S^{1}\right)=T^{2}$. Portanto a sequência 6.3 .1 se torna

$$
\left[S^{1} ; W\right] \stackrel{f^{*}}{\longleftarrow}\left[S^{1} \vee S^{1} ; W\right] \stackrel{j^{*}}{\longleftarrow}\left[T^{2} ; W\right] \stackrel{k^{\prime *}}{\longleftarrow}\left[S^{2} ; W\right] \stackrel{S f^{*}}{\longleftarrow}\left[S\left(S^{1} \vee S^{1}\right) ; W\right]
$$

Pelo resultado 5.19 do capítulo 3 de [22], [ $\left.S^{1} \vee S^{1} ; W\right]=\left[S^{1} ; W\right] \times\left[S^{1} ; W\right]$.

Portanto tomemos $[A]=\left(\left[A_{1}\right],\left[A_{2}\right]\right) \in\left[S^{1} ; W\right] \times\left[S^{1} ; W\right]$. Agora $f^{*}([A])=[A \circ f] \mathrm{e}$ afirmo que $f^{*}([A])=0$. Pois, suponha que $\left[A_{1}\right]$ é a classe que dá $x$ voltas em $S^{1} \subset W$ e $\left[A_{2}\right]$ dá $y$ voltas em $S^{1} \subset W$. A aplicação $f$ é representada por $a b a^{-1} b^{-1}$, onde $a$ significa que $f$ dá uma volta numa $S^{1}$ e $b$ que $f$ dá uma volta na outra $S^{1}$ e $a^{-1}$ e $b^{-1}$ são voltas no sentido contrário. Então $f^{*}([A])$ dará $x y x^{-1} y^{-1}$ voltas em $S^{1} \subset W$. Por propriedades de homotopia e sabendo-se que o caminho representado por $x y x^{-1} y^{-1}$ é homotópico ao caminho constante $\left(\pi_{1}(W) \simeq \mathbb{Z}\right.$ é abeliano). O que implica que $f^{*}([A])=0$.

Da mesma maneira vê-se que $S f^{*}=0$, por definição de suspensão de aplicação. Simplificando a sequência 6.3.2, temos:

$$
0 \stackrel{f^{*}}{\longleftarrow}\left[S^{1} \vee S^{1} ; W\right] \stackrel{j^{*}}{\longleftarrow}\left[T^{2} ; W\right] \stackrel{k^{\prime *}}{\longleftarrow}\left[S^{2} ; W\right] \stackrel{S f^{*}}{\longleftarrow} 0 .
$$

Pelo teorema [6.2.2, $\left[S^{2}, W\right]$ atua sobre $\left[T^{2}, W\right]$ segundo a ação definida em 6.2.5. O conjunto $\left[T^{2}, W\right]$ será caracterizado descrevendo-se esta ação e utilizando a sequência de 
Puppe-Barrat.

\section{Ação de $\left[S^{2}, W\right]$ em $\left[S^{2}, W\right] \subset\left[T^{2}, W\right]$}

Primeiramente algumas propriedades e observações.

Dada uma classe de homotopia $[h] \in\left[T^{2}, W\right]$, tal que $h_{\pi}: \pi_{1}\left(T^{2}\right) \rightarrow \pi_{1}(W)$ seja o homomorfismo nulo, então $[h] \in\left[S^{2}, W\right] \subset\left[T^{2}, W\right]$, onde $k^{*}\left(\left[S^{2} ; W\right]\right)$ é identificado à $\left[S^{2} ; W\right]$ já que $k^{*}$ é injetiva. Como $h_{\pi}=0$, então $h$ se levanta a uma aplicação $\widetilde{h}: T^{2} \rightarrow \widetilde{W}$, onde $\widetilde{W}$ é o espaço de recobrimento universal esquematizado anteriormente.

Defina uma aplicação $g: S^{2} \rightarrow W$ tal que $\widetilde{g}\left(S^{2}\right)=\widetilde{h}\left(T^{2}\right)$, onde $\widetilde{g}$ é o levantamento de $g$ ao recobrimento universal $\widetilde{W}$. Por definição $k^{* *}([g])=[h]$. Como $k^{\prime *}$ é injetiva, identificamos $[h]$ à $[g]$.

Sejam $f_{1}, f_{2} \in[T, W]$ tal que $\left(f_{1}\right)_{\pi}=\left(f_{2}\right)_{\pi}=0$, isto é, $\left[f_{1}\right],\left[f_{2}\right] \in\left[S^{2}, W\right]$, como descrito acima. Pelo lema 6.2.5 temos

$$
\left[d\left(f_{1}, f_{2}\right)\right]\left[f_{1}\right]=\left[f_{2}\right]
$$

Portanto dadas $[f] \in\left[S^{2}, W\right]$ e $\left[f_{1}\right] \in[T, W]$ tal que $\left(f_{1}\right)_{\pi}=0$, então existe $\left[f_{2}\right] \in[T, W]$ tal que $[f]=\left[d\left(f_{1}, f_{2}\right)\right]$. A construção de $f_{2}$ é da seguinte forma. Seja o toro dado pelo quadrado abaixo com os lados identificados

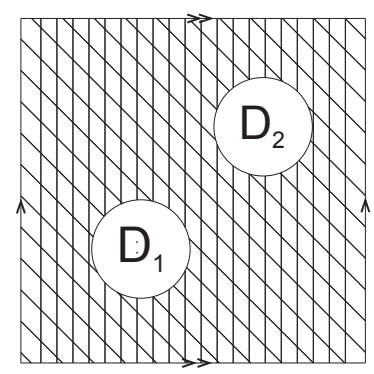

Então imagem de $f_{2}$ é dada por; a parte hachurada unida com os bordos de $\partial D_{1}$ e $\partial D_{2}$ é levada no ponto de wedge de $W, f_{2}(x)=f_{1}(x)$ se $x \in \operatorname{Int}\left(D_{1}\right)$ e $f_{2}(x)=f(x)$ se $x \in \operatorname{Int}\left(D_{2}\right)$. Logo $[f]\left[f_{1}\right]=\left[f_{2}\right]$.

Dessa forma, a ação sobre $\left[S^{2}, W\right]$ é trivial, no sentido que a imagem da ação de $\left[S^{2}, W\right]$ sobre $\left[S^{2}, W\right] \subset\left[T^{2}, W\right]$ é o próprio conjunto $\left[S^{2}, W\right] \subset\left[T^{2}, W\right]$. 
Geometricamente a ação é da seguinte forma: como $(f)_{\pi}=\left(f_{1}\right)_{\pi}=0$ então existem levantamentos $\widetilde{f}, \widetilde{f}_{1}: T^{2} \rightarrow \widetilde{W}$, onde $\widetilde{W}$ é o espaço de recobrimento de $W$, comutando os seguintes diagramas
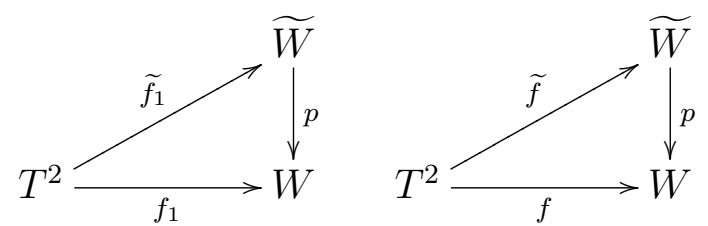

Suponha que as imagens de $\widetilde{f}, \widetilde{f}_{1}$ sejam respectivamente as área vermelha e azul abaixo,
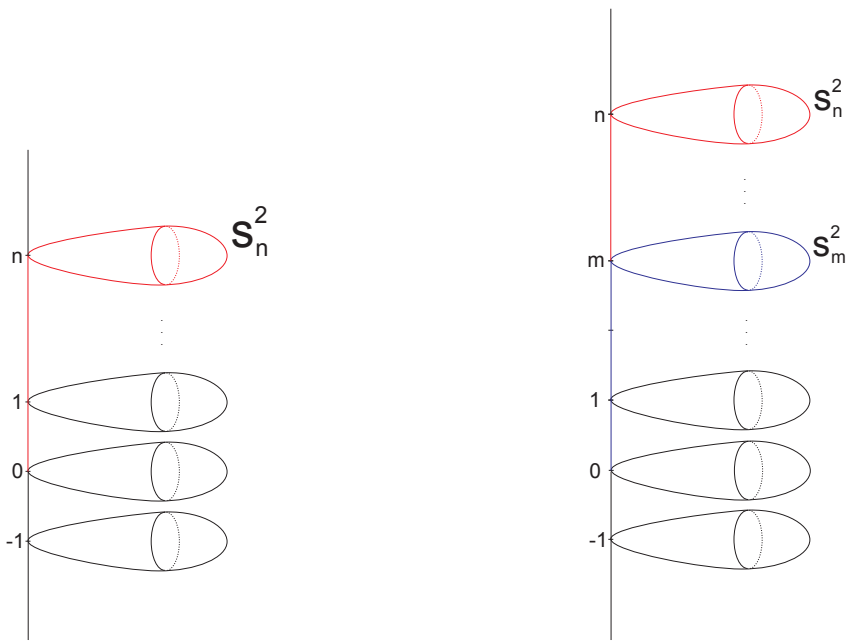

onde $\tilde{f}$ recobre $x$ vezes $S_{n}^{2}$ e $\tilde{f}_{1}$ recobre $x_{1}$ vezes $S_{m}^{2}$. Lembrando do exemplo 1, a ação de $[f]\left[f_{1}\right]$ terá imagem

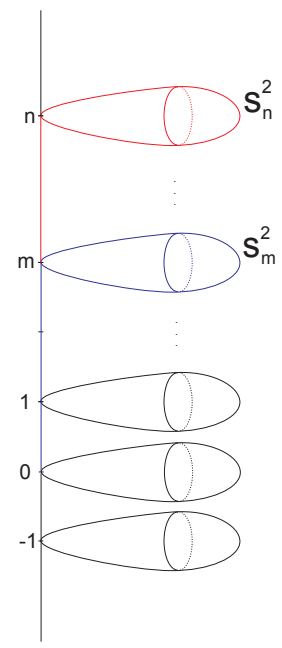

e recobre $x$ vezes $S_{n}^{2}$ e $x_{1}$ vezes $S_{m}^{2}$. Descrevendo algebricamente, temos as classes de homotopias $[f],\left[f_{1}\right] \in\left[S^{2}, W\right]=\pi_{2}(W) \cong \mathbb{Z}\left[t, t^{-1}\right]$ como polinômios e basta somar os 
polinômios, como feito na seção 1.2 .

Ação de $\left[S^{2}, W\right]$ em $\left[T^{2}, W\right] \backslash\left[S^{2} ; W\right]$

Sejam $f \in\left[S^{2}, W\right]$ e $f_{1} \in\left[T^{2}, W\right] \backslash\left[S^{2} ; W\right]$, isto é, $\left(f_{1}\right)_{\pi} \neq 0$. Primeiramente suponha que $f_{1}(T) \subset S^{1} \vee\left\{x_{0}\right\}$. De acordo com a ação $[f]\left[f_{1}\right]=\left[\Delta^{\prime} \circ\left(f \vee f_{1}\right) \circ \alpha^{\prime}\right]=[g]$, onde $\alpha^{\prime}$ definido como em 6.2.2. Temos, a menos de homotopia, $\left.f\right|_{S^{1} \vee S^{1}}=x_{0}$, então $\left.f_{1}\right|_{S^{1} \vee S^{1}}=\left.g\right|_{S^{1} \vee S^{1}}$. Logo $\left[d\left(f_{1}, g\right)\right]=[f]$ já que $\left[d\left(f_{1}, g\right)\right]\left[f_{1}\right]=[g]$.

Geometricamente a ação age da seguinte maneira: denote $\left(f_{1}\right)_{\pi}$ a induzida de $f_{1}$ no grupo fundamental. Sejam $(1,0),(0,1) \in \pi_{1}\left(T^{2}\right) \cong \mathbb{Z} \oplus \mathbb{Z}$ os geradores tais que $\left(f_{1}\right)_{\pi}(1,0)=x$ e $\left(f_{1}\right)_{\pi}(0,1)=y$, onde $x, y \in \pi_{1}(W) \cong \mathbb{Z}$ com $x y \neq 0$. Pois o caso $x y=0$ é a situação anterior, onde $\left(f_{1}\right)_{\pi}=0$. Como $f_{1}\left(T^{2}\right) \subset S^{1} \vee\left\{x_{0}\right\}$, a menos de homotopia $f_{1}$ é dada esquematicamente como no quadrado abaixo, que é identificado no toro $T^{2}$,

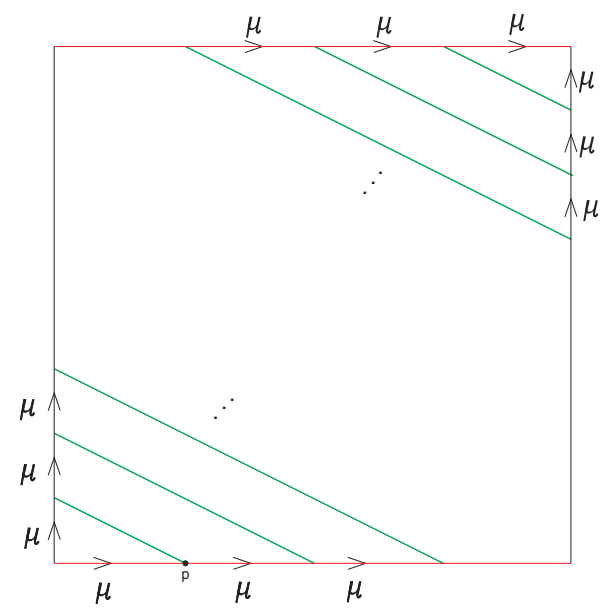

onde os lados verticais estão divididos em $x$ partes iguais e os lados horizontais do quadrado estão divididos em $y$ partes iguais. Assim cada uma destas partes, denotado por $\mu$, é levado por $f_{1}$ em $S^{1} \subset W$ recobrindo uma vez. A imagem de toda a parte verde é o ponto de wedge de $W$.

Sobre este toro selecionamos um disco $D$, como no esquema abaixo 


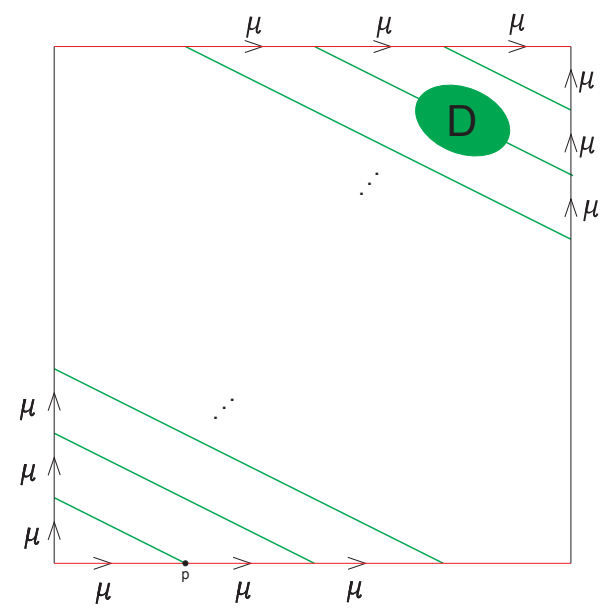

Agora a ação de $[f]$ sobre $\left[f_{1}\right]$ resultará na classe $[g] \in\left[T^{2} ; W\right]$. Esta aplicação $g$ é dada tomando um outro disco $D_{1}$ no interior do disco construído anteriormente, o qual será o domínio de $f$ como segue,

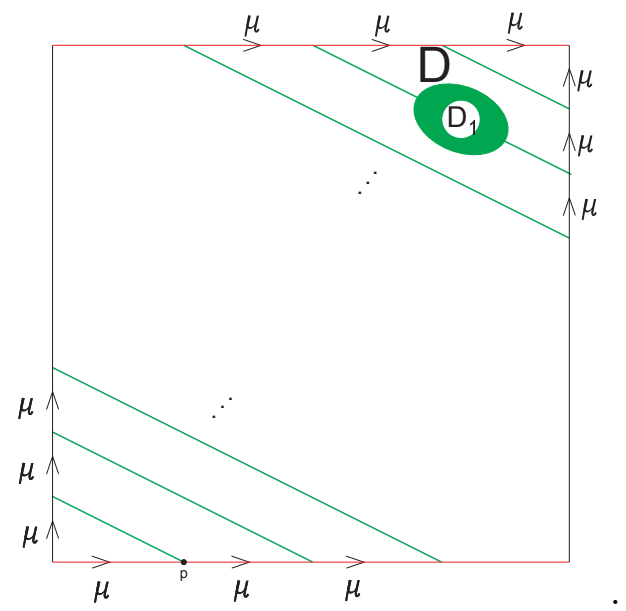

Assim como antes, $g$ leva $\mu$ em $S^{1} \subset W$, cobrindo uma vez ,e toda a parte verde é levado no ponto $\left\{x_{0}\right\} \in S^{1} \vee\left\{x_{0}\right\}$ e, no disco $D_{1}, g$ é definida como $f$. Isto é,

$$
g(x)=\left\{\begin{array}{lll}
f_{1}(x) & \text { se } & x \in T \backslash D \\
x_{0} & \text { se } & x \in D \backslash \stackrel{\circ}{D}_{1} \\
f(x) & \text { se } & x \in \stackrel{\circ}{D}_{1}
\end{array}\right.
$$

Por construção $g$ é contínua é satisfaz $\left[d\left(f_{1}, g\right)\right]\left[f_{1}\right]=[g]$.

No caso em que $f_{1} \in\left[T^{2}, W\right] \backslash\left[S^{2} ; W\right]$ é sobrejetora, temos $\left[f_{1}\right]=[g][f]$, onde $[f]$ e $[g]$ são construídas a partir de $f_{1}$ de forma que $[g] \in\left[S^{2} ; W\right]$ e $[f] \in\left[T^{2}, W\right] \backslash\left[S^{2} ; W\right]$ 
tal que $f\left(T^{2}\right)=S^{1} \vee\left\{x_{0}\right\}$. Usando as mesmas técnicas da seção 1.2, mostra-se que $\left(f_{1}\right)^{-1}\left(W \backslash S^{1} \vee\left\{x_{0}\right\}\right)$ é uma união finita disjunta de discos $\bigsqcup_{i=1}^{n} D_{i}$.

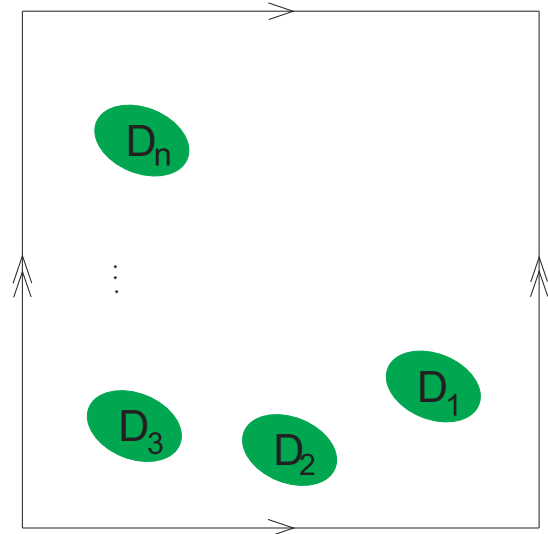

A aplicação $f$ é construída de forma que $f(x)=f_{1}(x)$ para todo $x \in T^{2} \backslash \bigsqcup_{i=1}^{n} D_{i}$ e $f(x)=x_{0}$ para $x \in \bigsqcup_{i=1}^{n} D_{i}$. Para cada disco $D_{i}$ podemos considerar uma aplicação $g_{i}$ : $S_{2} \rightarrow W$ dada por $g_{i}(x)=f_{1}(x)$, onde $x \in D_{i}$. Então considere a aplicação $g: S^{2} \rightarrow W$ por $[g]=\left[g_{1}\right] \ldots\left[g_{n}\right]$. Portanto a função $f_{1}$ nada mais é do que a ação de $g$ sobre $f$.

A ação no conjunto $\left[T^{2} ; W\right]$, descreve que este conjunto é uma união disjunta de classes de equivalências de homotopia da seguinte maneira

$$
\left[T^{2} ; W\right]=\bigsqcup_{(x, y) \in \mathbb{Z} \oplus \mathbb{Z}}\left[S^{2} ; W\right]_{(x, y)}
$$

onde $\left[S^{2} ; W\right]_{(x, y)}$ é a ação de $\left[S^{2} ; W\right]$ na classe de homotopia $[f]$ tal que $f_{\pi}(1,0)=x$ e $f_{\pi}(0,1)=y$.

Analogamente pode-se caracterizar o conjunto de classes de homotopias $[S, W]$, onde $S$ é uma superfície compacta, conexa, orientável e sem bordo.

Neste caso, $f^{*}$ e $S f^{*}$ são aplicações nulas também e as classes de homotopias de $[S, W]$ é caracterizada pela ação de classes de homotopias de $\left[S^{2}, W\right]$ em aplicações de $S$ em $S^{1}$.

É conhecido que o conjunto de classes de homotopias de aplicações de $S$ em $S^{1}$ é 
$H^{1}(S, \mathbb{Z})$ a cohomologia de $S$ com coeficientes em $\mathbb{Z}$ no nível 1 . Portanto temos uma bijeção entre $[S ; W]$ e $\bigsqcup_{z \in H^{1}(S, \mathbb{Z})}\left[S^{2} ; W\right]_{z}$.

Quando temos $S$ uma superfície compacta, conexa, não orientável e sem bordo, é um pouco diferente. Por exemplo se considerarmos aplicações $f: \mathbb{R} P^{2} \rightarrow S^{2} \subset W$, pelo Corolário 16 (pág. 432) de [20] $\left[\mathbb{R} P^{2}, S^{2}\right] \simeq \mathbb{Z}_{2}$.

Seja $S=\mathbb{R} P_{g}^{2}$, então $\mathbb{R} P_{g}^{2}$ é obtida pela colagem $C S^{1} \cup_{f} S_{1}^{1} \vee \ldots \vee S_{g}^{1}$, onde $X=S^{1}$, $Y=S_{1}^{1} \vee \ldots \vee S_{g}^{1}$ e $f: X \rightarrow Y$ é a aplicação contínua caracterizada pela palavra $a_{1}^{2} a_{2}^{2} \ldots a_{g}^{2}$, onde $a_{i}^{2}$ significa que a imagem da $f$ dá duas voltas em $S_{i}^{1}$.

Pelo resultado 5.19 do capítulo 3 de [22], $\left[S_{1}^{1} \vee \ldots \vee S_{g}^{1} ; W\right]=\left[S_{1}^{1} ; W\right] \times \ldots \times\left[S_{g}^{1} ; W\right]$. Se $[A]=\left(\left[A_{1}\right],\left[A_{2}\right], \ldots,\left[A_{g}\right]\right) \in\left[S_{1}^{1} ; W\right] \times \ldots \times\left[S_{g}^{1} ; W\right]$ tal que $\left[A_{i}\right]$ é a classe que dá $x_{i}$ voltas em $S^{1} \subset W$. Então $f^{*}([A])$ dará $x_{1}^{2} \ldots x_{g}^{2}$ voltas em $S^{1} \subset W$.

Da mesma maneira se $[B] \in\left[S_{1}^{2} \vee \ldots \vee S_{g}^{2} ; W\right]$, então $\left[S_{1}^{2} \vee \ldots \vee S_{g}^{2} ; W\right]=\left[S_{1}^{2} ; W\right] \times$ $\ldots \times\left[S_{g}^{2} ; W\right]$ e $[B]=\left(\left[B_{1}\right], \ldots,\left[B_{g}\right]\right)$, onde cada $\left[B_{i}\right]$ é caracterizado pelo grau. Assim se $b_{i}$ é grau de cada $\left[B_{i}\right]$, então $S f^{*}([B])$ terá grau igual a $b_{1}^{2} \ldots b_{g}^{2}$.

Portanto $f^{*}$ e $S f^{*}$ é a multiplicação por dois em cada coordenada, desde que $\left[S_{1}^{2} \vee \ldots \vee\right.$ $\left.S_{g}^{2} ; W\right]=\left[S_{1}^{2} ; W\right] \times \ldots \times\left[S_{g}^{2} ; W\right] \simeq \underbrace{\mathbb{Z} \oplus \ldots \oplus \mathbb{Z}}_{g-\text { vezes }} \simeq\left[S_{1}^{1} ; W\right] \times \ldots \times\left[S_{g}^{1} ; W\right]=\left[S_{1}^{1} \vee \ldots \vee S_{g}^{1} ; W\right]$.

Neste caso a sequência de Puppe-Barrat é

$$
\left[S^{1} ; W\right] \stackrel{f_{*}^{*}=\bullet 2}{\longleftarrow}\left[S_{1}^{1} \vee \ldots \vee S_{g}^{1} ; W\right] \stackrel{j^{*}}{\longleftarrow}\left[\mathbb{R} P_{g}^{2} ; W\right] \stackrel{k^{\prime *}}{\longleftarrow}\left[S^{2} ; W\right] \stackrel{S f^{*}=\bullet 2}{\longleftarrow}\left[S_{1}^{2} \vee \ldots \vee S_{g}^{2} ; W\right]
$$

onde $\bullet 2$ significa que é a multiplicação por 2 .

Denotemos por $\overline{\left[S^{2} ; W\right]} \simeq \mathbb{Z}_{2}$ o conjunto $\frac{\left[S^{2} ; W\right]}{\operatorname{Im}\left(S f^{*}\right)}$.

Portanto o conjunto de classes de homotopia $\left[\mathbb{R} P_{g}^{2} ; W\right]$ é descrito como o seguinte

$$
\left[\mathbb{R} P_{g}^{2} ; W\right]=\bigsqcup_{z \in H^{1}\left(\mathbb{R} P_{g}^{2}, \mathbb{Z}\right)} \overline{\left[S^{2} ; W\right]}
$$




\section{Conclusão}

As classes de homotopia do toro em $W$ foram caracterizadas.

No caso $S$ ser uma superfície compacta, conexa, sem bordo e orientável as classes de homotopias de $[S, W]$ é caracterizada pela ação de classes de homotopias de $\left[S^{2}, W\right]$ em aplicações de $S$ em $S^{1}$.

É conhecido que o conjunto de classes de homotopias de aplicações de $S$ em $S^{1}$ é $H^{1}(S, \mathbb{Z})$ a cohomologia de $S$ com coeficientes em $\mathbb{Z}$ no nível 1 . Portanto temos uma bijeção entre $[S ; W]$ e $\bigsqcup_{z \in H^{1}(S, \mathbb{Z})}\left[S^{2} ; W\right]_{z}$.

Já no caso de $S$ ser uma superfície compacta, conexa, sem bordo e orientável as classes de homotopias de $[S ; W]$ é caracterizada pela ação das classes de homotopias de $\overline{\left[S^{2}, W\right]}$ em aplicações de $S$ em $S^{1}$. Portanto temos uma bijeção entre $[S ; W]$ e $\bigsqcup_{z \in H^{1}(S, \mathbb{Z})} \overline{\left[S^{2} ; W\right]}$.

Observe que para qualquer superfície compacta, conexa e sem bordo $S$ a representação de classes de homotopias de aplicações de $f: S \rightarrow W$ é dada a partir do polinômio associado a classe $p_{[f]}(t)$ e pelo gerador da imagem da induzida em grupo fundamental $<l>=f_{\pi}\left(\pi_{1}(S)\right)$, considerando a aplicação especial construída a partir deste polinômio e deste número natural.

Se considerarmos $S=S^{2}$, cada aplicação especial representa uma única classe de homotopia de $\left[S^{2} ; W\right]$. Logo existe uma bijeção entre aplicações especiais (que esta em bijeção com os polinômios associados) e o conjunto $\left[S^{2} ; W\right]$.

Já, por exemplo, no caso se $S=T^{2}$, então existem menos aplicações especiais do que classes de homotopias de $\left[T^{2} ; W\right]$. Pois, como vimos, o conjunto $\left[T^{2} ; W\right]$ está em bijeção com $\bigsqcup_{(x, y) \in \mathbb{Z} \oplus \mathbb{Z}}\left[S^{2} ; W\right]_{(x, y)}$, pela sequência de Puppe-Barratt, e as aplicações especiais, que são caracterizadas pelos polinômios associado e por número natural, está em bijeção com o conjunto $\bigsqcup_{z \in \mathbb{N}} \operatorname{Pol}_{z}$, onde $P o l=\left\{a_{0}+\ldots+a_{n} t^{n} ; n \geq 0\right.$ e $\left.a_{i} \in \mathbb{N}\right\} \subset \mathbb{Z}\left[t, t^{-1}\right]$ e o conjunto Pol esta em bijeção com $\left[S^{2} ; W\right]$. Portanto para cada aplicação especial existem mais de uma classe de homotopia de $\left[T^{2} ; W\right]$ que são representadas pela mesma aplicação especial.

Isto ocorre pois para obter a aplicação especial mudamos a classe de homotopia da aplicação usando homeomorfismo do domínio (quando é feita a escolha de base de $\pi_{1}(S)$ 
ou em certos casos de $\left.H_{1}(S, \mathbb{Z})\right)$. Note que a configuração de raízes de $f: S \rightarrow W$ não muda se compomos $f$ com homeomorfismo $h: S \rightarrow S$, com isso, estudar as raízes da classe $[f]$ é equivalente a estudar raízes de $[f \circ h]$, isto explica a diminuição da quantidade de classes de homotopia estudada. 


\section{Referências Bibliográficas}

[1] Aniz, C., Raízes de funções de um complexo em uma variedade, tese de doutorado do ICMC-USP, 2002.

[2] Barcus, W. D., Barrat, M. G., On the homotopy classification of the extensions of a fixed map, Trans. Amer. Math. Soc., 88, 1958, 57-74.

[3] Barratt, M. G., Track groups I, Proc. London Math. Soc., 5, (1955), 71-106.

[4] Barratt, M. G., Track groups II, Proc. London Math. Soc., 5, (1955), 285-329.

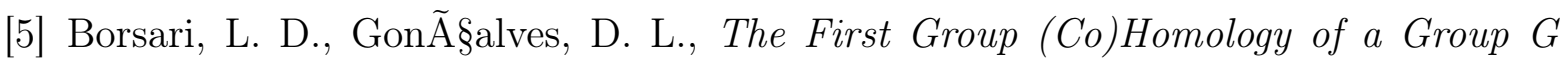
with Coefficients in Some G-Modules Quaest. Math., 31, (2008), 89-100.

[6] Brooks, R., Roots of mappings from manifolds, Fixed Point Theory Appl., 4, (2004), 273-307.

[7] Browder, W., Surgery on simple-connected manifolds, Ergebnisse der mathematik und ihrer grenzgebiete, Band 65, Springer-Verlag Berlin Heidelberg New York, 1972.

[8] Brown, R. F., Furi, M., Górniewics, L., Jiang, B., Handbook of Topological Fixed Point Theory, Springer-Dordrecht, 2005.

[9] Dold, A., Lectures on Algebraic Topology, Springer-Verlag Berlin Heidelberg New York, 1980.

[10] Fenille, M. C., Raízes de aplicações de complexos 2-dimensionais em superfícies fechadas, tese de doutorado do ICMC-USP, 2009. 
[11] Fox, R. H., On topologies for function spaces, Bull. Amer. Soc., 51, (1945), 429-432.

[12] Jackson, J. R., Spaces of mappings on topological products with applications to homotopy theory, Proc. Amer. Math. Soc., 3, (1952), 327-333.

[13] Davis, J. F., Kirk, P., Lectures Notes in Algebraic Topology, American Mathematical Society, 55-XX, 1991.

[14] Milnor, J., Lectures on the h-Cobordism Theorem, Princeton University Press., 1965.

[15] Munkres, J. R., Elementary Differential Topology, Princeton University Press, Study $\mathbf{5 4}, 1966$.

[16] Munkres, J. R., Elements of Algebraic Topology, Westview Press, 1984.

[17] Pajitnov, A. Circle-valued Morse Theory, Walter de Gruyter 32, 2006.

[18] Puppe, D., Homotopiemengen und ihre induzierten Abbildungen I, Math. Z., 69, (1958), 299-344.

[19] Rourke, C. P., Sanderson, B.J., Introduction to Piecewise-Linear Topology, SpringerVerlag Berlin Heidelberg New York, 1972.

[20] Spanier, E. H., Algebraic Topology, Springer-Verlag Berlin Heidelberg New York, 1966.

[21] Switzer, R. M., Algebraic Topology- Homotopy and Homology, Springer-Verlag Berlin Heidelberg New York, 1975.

[22] Whitehead, G. W., Elements of Homotopy Theory, Springer-Verlag Berlin Heidelberg New York, 1978. 